\title{
Hot (3)
}

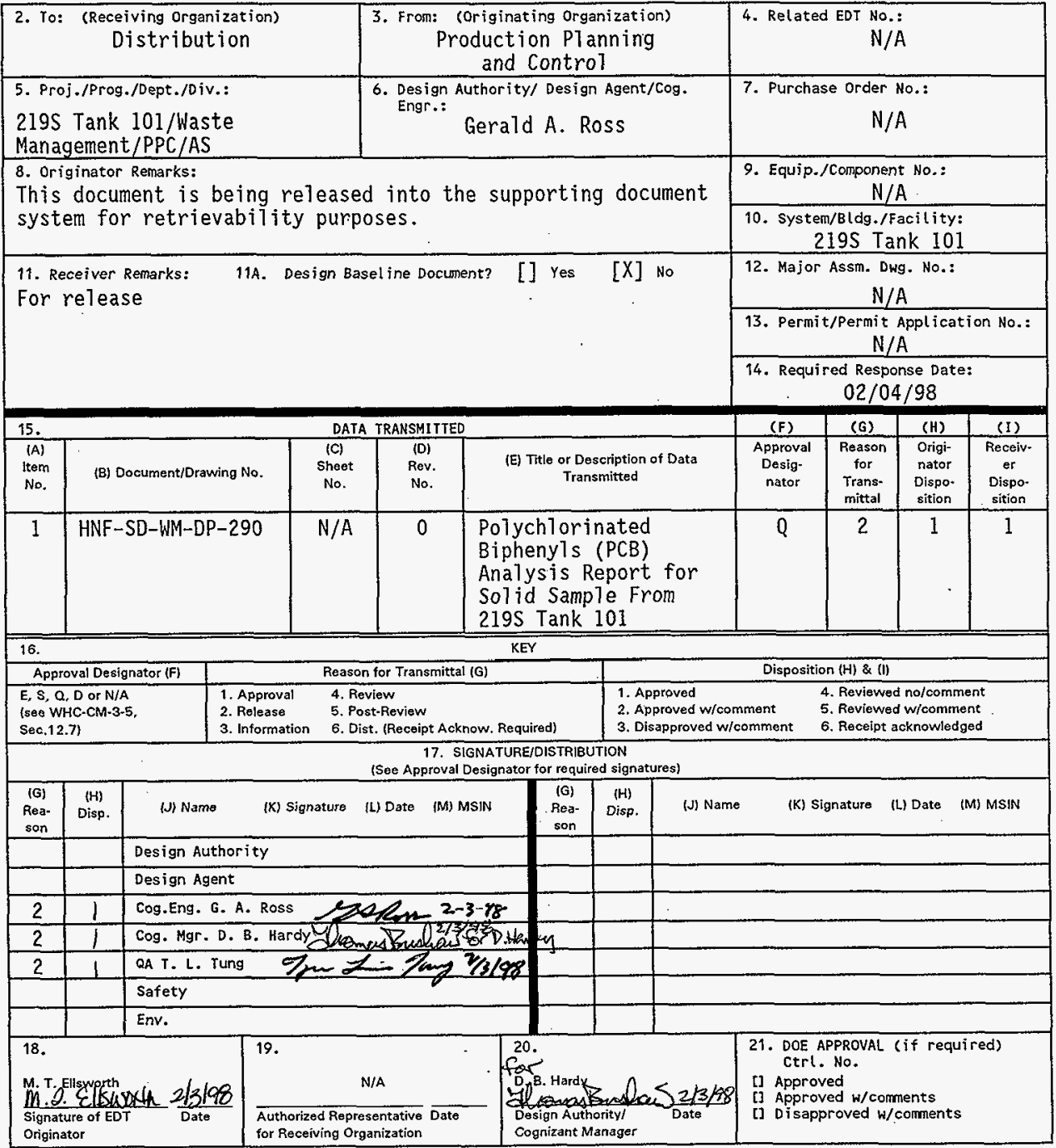

BD-7400-172-2 (05/96) GEF097 


\section{Polychlorinated Biphenyls (PCB) Analysis Report for Solid Sample from 219S Tank 101}

\section{Gerald A. Ross}

Waste Management of Hanford, Inc., Richland, WA 99352

U.S. Department of Energy Contract DE-AC06-96RL13200

EDT/ECN: EDT-623140

Org Code: 31133

B\&R Code: EW 3120074 Total Pages: $/ 29$

Key Words: Polychlorinated, Biphenyls, PCB, Analysis, Report, for Solid Samples, 219S, Tank 101, 101

Abstract: $\mathrm{N} / \mathrm{A}$

TRADENARK DISCLAIMER. Reference herein to any specific commercial product, process, or service by trade name, trademark, manufacturer, or otherwise, does not necessarily constitute or imply its endorsement, recomendation, or favoring by the United states Government or any agency thereof or its contractors or subcontractors.

Printed in the United States of America. To obtain copies of this document, contact: Document Control Services, P.0. Box 950, Mailstop H6-08, Richland WA 99352, Phone (509) 372-2420; Fax (509) 376-4989.
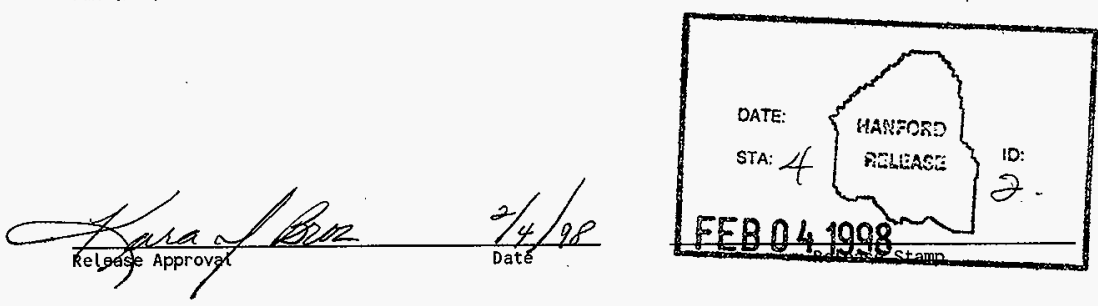

Approved for Public Release 
HNF-SD-WM-DP-290, REV. 0

ANALYTICAL SERVICES

\title{
POLYCHLORINATED BIPHENYLS (PCB) ANALYSIS REPORT FOR SOLID SAMPLE FROM 219S TANK 101
}

\author{
Project Coordinator: Gerald A. Ross \\ Prepared for the U.S. Department of Energy \\ Office of Environmental Restoration \\ and Waste Management \\ by \\ 222-S Laboratory \\ Waste Management of Hanford, Inc. \\ P.0. Box 700 \\ Rich 1 and, Washington
}


HNF-SD-WM-DP-290, REV. 0

THIS PAGE INTENTIONALLY LEFT BLANK 
HNF-SD-WM-DP-290, REV. 0

TABLE OF CONTENTS

Narrative ........................ 1 Appendix A $(L A-523-138) \ldots \ldots \ldots \ldots$

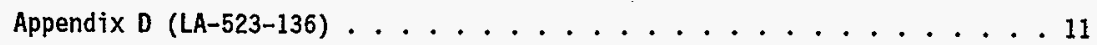
Request for Special Analysis ................. . . 13.1 CLP-Like Forms Summary Result Forms . . . . . . . . . . . . . 14 Extraction Benchsheet ................... 20

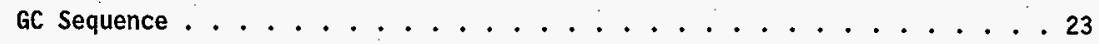

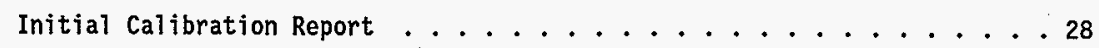

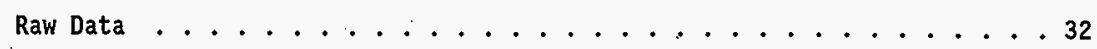
Continuing Calibration Verification .............. 114

This document consists of pages 1 through 119. Pages ii, 2, 9, 12, 13.2, 15, $21,24,29,33$ and 115 were intentionally left blank. 
HNF-SD-WM-DP-290, REV. 0

\section{NARRATIVE}


HNF-SD-WM-DP-290, REV. 0

THIS PAGE INTENTIONALLY LEFT BLANK 
POLYCHLORINATED BIPHENYLS (PCB) ANALYSIS REPORT

FOR SOLID SAMPLE FROM 219S TANK 101

\section{SAMPLE ANALYSIS REPORTED}

One waste sample that was obtained with solids from tank 101 of 2195 via a peristaltic pump equipped with a stainless steel tube and Norprene tubing (Pthalate free) was obtained in a glass jar with teflon lid was analyzed (with duplicate, matrix spike, and matrix spike duplicate) for PCBs as Aroclor mixtures by the Inorganic/Organic Chemistry Group. A soxhlet extraction procedure was used for extraction of the Aroclors from the sample. Analysis was performed using dual column confirmation gas chromatography/electron capture detection (GC/ECD). Extraction follows closely method $3540 \mathrm{C}$ of SW846 , analysis fol]ows SW-846 method 8082 .

A cross reference of laboratory sample number to the customer identification is given in the following table:

\begin{tabular}{|c|l|}
\hline \multicolumn{1}{|c|}{ Sample Number } & \multicolumn{1}{|c|}{ Customer ID } \\
\hline S975000235 & 101LW Organic Tests \\
\hline
\end{tabular}

SAMPLE DESCRIPTION, HANDLING, AND PREPARATION

The sample was dark brown in color and had the consistency of soft peanut butter. After limited stirring four one gram aliquots were taken from the parent sample jar for analysis. Due to limited sample and radiation dose concerns a nominal one gram of sample was used for each analysis. Extraction of the samples was completed on January 15,1998 in a radiological fume hood.

Preparation Procedure: LA-523-138 Rev. A0

Preparation Location: 222-S Laboratory, 200W Area, Hanford Site, Room 4P

Preparation Type: Soxhlet Extraction using methylene chloride, Kuderna-

Danish concentration with solvent exchange to hexane and cleanup by sulfuric acid.

Sample Extract Storage: 4 degrees Celsius in darkness

ANALYSIS METHOD

GC/ECD Procedure: LA-523-136 Rev. A0

GC/ECD instrumentation: HP-5890 Series II gas chromatograph with dual oncolumn injection, XTI-5 and DB-608 columns, and duaT EC Detectors, (property number AL 11217).

Location: 222-S Laboratory, Room 4TUV

The analysis was performed on January $16 \& 23,1997$ on the extracts obtained on January $15,1998$. 


\section{HNF-SD-WM-DP-290 REV. 0}

\section{QUALITY CONTROL}

Due to limited sample amount and radiological issues the extraction portion of the procedure is scaled down from SW-846 method 3540C. Appendix A of LA-523138 details the deviations to method $3540 \mathrm{C}$ that are contained in the procedure. Analysis follows method 8082 of SW-846. Deviations from method 8082 are detailed in appendix D of LA-523-136. Nominally. Ig of sample was extracted per analytical result.

Five point calibrations were run for the following aroclors: Aroclor 1248. Aroclor 1254 and Aroclors 1016/1260. Concentration ranges went from $20 \mathrm{ng} / \mathrm{mL}$ to $400 \mathrm{ng} / \mathrm{mL}$. Single point calibrations were performed for Aroclors 1221 , 1232, and 1242. The sample analysis was bracketed with a second mid-level calibration point of Aroclors 1016/1260 analyzed at the end of the run sequence. An instrument blank was analyzed at the end of the calibration prior to the sample analysis.

Quantitation was performed using a primary column and the secondary column was used for confirmation of identification. A detailed description of the specific procedure requirements is available in the procedure. The following summarizes the $Q C$ requirements and adherence:

Table I.

\begin{tabular}{|c|c|}
\hline QC Parameter & Comments \\
\hline Target Compounds & $\begin{array}{l}\text { Detection levels are based on sample size and the } \\
\text { determination of MDLs from multiple injections of a } \\
\text { low standard. Met requirements. (Page 16-19). }\end{array}$ \\
\hline Surrogate Recoveries & $\begin{array}{l}\text { Surrogate spiking was performed on all samples using } \\
\text { TCX and DCB. No control limits have been estab- } \\
\text { lished for these compounds in this matrix. A summary } \\
\text { of the surrogate recoveries is provided in Table II. } \\
\text { All Surrogate recoveries were within acceptable } \\
\text { administrative limits (see Table II. Page 6). }\end{array}$ \\
\hline $\begin{array}{l}\text { Matrix Spike } \\
\text { Recovery }\end{array}$ & $\begin{array}{l}\text { A matrix spike and matrix spike duplicate using } \\
\text { Aroclors } 1016 \text { and } 1260 \text { were performed. Due to the } \\
\text { level of Aroclors in the sample. Spike amounts were } \\
\text { less thatn the variability in the sample result. } \\
\text { Hence, spike amounts and recoveries are invalid. } \\
\text { (see Table III, page } 6.1 \text {, and discussion on page 5). }\end{array}$ \\
\hline Method Blank Summary & $\begin{array}{l}\text { A method blank was extracted and met requirements. } \\
\text { (page 78). }\end{array}$ \\
\hline $\begin{array}{l}\text { Initial Method } \\
\text { Detection Limit } \\
\text { Determination }\end{array}$ & $\begin{array}{l}\text { Multiple extraction and analysis of a low } \\
\text { concentration standard. Met requirements. (For } 2 \mathrm{~g} \\
\text { sample the MDL for } 1248=3.71 \mathrm{ug} / \mathrm{kg} \text {. documented in } \\
\text { files). }\end{array}$ \\
\hline
\end{tabular}




\begin{tabular}{|c|c|}
\hline Initial Calibration & $\begin{array}{l}\text { Five point calibrations were performed for Aroclors } \\
1248,1254 \text {, and } 1016^{*} / 1260^{*} \text {. Concentrations ranged } \\
\text { from } 20 \mathrm{ng} / \mathrm{mL} \text { to } 400 \mathrm{ng} / \mathrm{mL} \text {. Single point calibrations } \\
\text { were performed for Aroclors 1221. 1232, and } 1242 \text { at } \\
100 \text { or } 200 \mathrm{ng} / \mathrm{mL} \text {. Some levels with prep error were } \\
\text { omitted. ( } *=5 \text { points used). Met requirements. } \\
\text { (Initial calibration report, page } 30-31 \text { ). }\end{array}$ \\
\hline $\begin{array}{l}\text { Carry-over } \\
\text { evaluation. }\end{array}$ & $\begin{array}{l}\text { An instrument blank was run after the calibration } \\
\text { standards. No carry-over was detected. Met } \\
\text { requirements (pages } 73,92,112 \text { ). }\end{array}$ \\
\hline $\begin{array}{l}\text { Continuing } \\
\text { Calibration } \\
\text { Verifications }\end{array}$ & $\begin{array}{l}\text { Initial calibration was performed during the sample } \\
\text { sequence. Aroclors } 1016 / 1260 \text { were used to assess } \\
\text { ongoing instrument performance after the sample was } \\
\text { analyzed on it. Percent Differences were }+27.8 \% \text { and } \\
+15.4 \% \text { respectively. The } 1016 \text { recovery was high, } \\
\text { but otherwise met requirements. Later CCV confirmed } \\
\text { instrument was ok by } 95.6 \% \text { and } 81.5 \% \text { recovery (page } \\
\text { 116). }\end{array}$ \\
\hline $\begin{array}{l}\text { Laboratory Control } \\
\text { Sample }\end{array}$ & $\begin{array}{l}\text { A matrix spike blank was used as a Laboratory } \\
\text { Control Sample to assess method performance. This } \\
\text { contained Aroclors } 1016 \text {, and } 1260 \text {. Recoveries of } \\
\text { A1016/A1260 were } 91.8 \% \text { and } 92.9 \% \text {. respectively. This } \\
\text { met the requirements (page } 82 \text { ). }\end{array}$ \\
\hline
\end{tabular}

Laboratory bench sheets, data, chromatograms, and reports are maintained in room $3 \mathrm{~B}$ at the 222-S Laboratory. The initiai calibration information and data is located on the HP UNIX system. Chemsys1.

\section{RESULTS / DISCUSSION}

The aroclors 1016, 1248, 1254, and 1260 were observed in the sample, above the lower quantitation limit. The amounts are reported in the attached result summary forms. The total number of PCBs quantified in sample 59750000235 as Aroclors is $14.9 \mathrm{mg} / \mathrm{Kg}$ and $16.48 \mathrm{mg} / \mathrm{Kg}$ for the sample and duplicate. respectively. The average total PCBs as Aroclors value is $15.7 \mathrm{mg} / \mathrm{Kg}$.

The Aroclor concentrations found in the sample were much higher than the spike levels (200ppb), hence the MS/MSD amounts are smaller than the variability in the sample results. Hence, the MS/MSD values are invalid due to the spike concentration was much less than $25 \%$ of the sample concentration. Rerun was not possible due to insufficient amount of sample left. However. The Laboratory Control Spike recoveries were very good.

Surrogate spiking was performed on all samples using Tetrachloro-m-xylene (TCX) and Decachlorobiphenyl (DCB) and is summarized in Table II. No control limits have been established for these compounds in this matrix. USEPA advisory limits set at $50-150 \%$ further indicate surrogate recoveries for these samples is acceptable. 


\section{HNF-SD-WM-DP-290 REV. 0}

Table II.

SURROGATE RECOVERIES (\%)

\begin{tabular}{|c|c|c|}
\hline Sample & TCX (\%) & DCB (\%) \\
\hline Inst. Blank & 125.8 & 144.8 \\
\hline Meth. Blank & 61.59 & 107.9 \\
\hline LCS & 52.93 & 69.21 \\
\hline S97S0000235 & 114.70 & 94.860 \\
\hline Inst. Blank & 109.0 & 73.46 \\
\hline S97S0000235D & 63.970 & $52.88 D$ \\
\hline S97S0000235MS & 137.10 & 100.20 \\
\hline S97S0000235MSD & 69.910 & 51.090 \\
\hline Inst. Blank & 114.8 & 96.83 \\
\hline CCV & 133.0 & 145.2 \\
\hline
\end{tabular}

(A) results are within advisory limits of 50-150\%, the 20-150\% range printed in the surrogate spike recovery reports is not being used as criteria.

Diluted samples are flagged with "D") 
TABLE III.

HNF-SD-WM-DP-290 REV. 0

SOIL PCB COMPOUNDS MATRIX SPIKE/MATRIX SPIKE DUPLICATE RECOVERY

Lab Name: 222-S

Lab code:

Case No.:
Contract:

SAS No.:

SDG No.: 012398

Matrix Spike - EPA Sample No.: S97S00235 Level(low/med) LOW

\begin{tabular}{|c|c|c|c|c|c|}
\hline COMPOUND & $\begin{array}{l}\text { SPIKE } \\
\text { ADDED } \\
(u g / K g)\end{array}$ & $\begin{array}{c}\text { SAMPLE } \\
\text { CONCENTRATION } \\
(\mathrm{ug} / \mathrm{Kg})\end{array}$ & $\begin{array}{c}\text { MS } \\
\text { CONCENTRATION } \\
(u g / K g)\end{array}$ & $\begin{array}{c}\text { MS } \\
\% \\
\text { REC \# }\end{array}$ & $\begin{array}{l}\text { QC. } \\
\text { LIMITS } \\
\text { REC. }\end{array}$ \\
\hline $\begin{array}{l}\text { Aroclor }-1016 \\
\text { Aroclor }-1260\end{array}$ & $\begin{array}{l}192 \\
192\end{array}$ & $\begin{array}{l}1490 \\
1710\end{array}$ & $\begin{array}{l}1140 \\
2490\end{array}$ & $\begin{array}{r}0 * \\
407 *\end{array}$ & $\begin{array}{l}50-150 \\
50-150\end{array}$ \\
\hline
\end{tabular}

\begin{tabular}{|c|c|c|c|c|c|c|}
\hline & $\begin{array}{l}\text { SPIKE } \\
\text { ADDED }\end{array}$ & $\begin{array}{c}\text { MSD } \\
\text { CONCENTRATION }\end{array}$ & $\begin{array}{c}\text { MSD } \\
\%\end{array}$ & 0 & \multicolumn{2}{|c|}{ QC LIMITS } \\
\hline COMPOUND & $(\mathrm{ug} / \mathrm{Kg})$ & (ug/Kg) & REC \# & RPD \# & RPD & REC. \\
\hline $\begin{array}{l}\text { Aroclor }-1016 \\
\text { Aroclor }-1260\end{array}$ & $\begin{array}{l}196 \\
196\end{array}$ & $\begin{array}{l}1450 \\
2340\end{array}$ & $\begin{array}{r}0 * \\
322 *\end{array}$ & $\begin{array}{c}\mathrm{N} / \mathrm{A} * \\
23\end{array}$ & $\begin{array}{l}40 \\
40\end{array}$ & $\begin{array}{l}50-150 \\
50-150\end{array}$ \\
\hline
\end{tabular}

\# Column to be used to flag recovery and RPD values with an asterisk

* Values outside of QC limits

RPD: 1 out of 2 outside limits

Spike Recovery: 4 out of 4 outside limits

COMMENTS: Failed spike due to $<25 \%$ of sample result, see discussion in the narrative. Ignore the spike rec. \% calculated in the forms on pg 101, and pg 106 because of the error in value in the software. 
HNF-SD-WH-DP-290 REV. 0

File: C: \WPDATA

ANALYS HAthor DATE 2/2/98 REVIEWER Yhomes Bubleas DATE 2/3/98 
HNF $-S D-W M-D P-290$, REV. 0

APPENDIX A

LA-523-138 
HNF-SD-WM-DP-290, REV. 0

THIS PAGE INTENTIONALLY LEFT BLANK 


\section{Appendix A \\ Comparison to SW-846}

Method 3540C, Soxhlet Extraction, Revision 3, December 1996

\begin{tabular}{|c|c|c|c|}
\hline 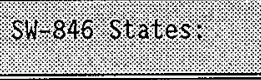 & Procedure States? & Troe of Ghanger: & $\begin{array}{l}\text { Reason ior } \\
\text { Changer }\end{array}$ \\
\hline $\begin{array}{l}\text { Section } 7.2 \text { : Use } \\
5 \text { to } 10 \text { grams of } \\
\text { sample }\end{array}$ & $\begin{array}{l}\text { Step 9.5: Use } 2 \\
\text { to } 10 \text { grams of } \\
\text { sample }\end{array}$ & $\begin{array}{l}\text { Deviation- allows } \\
\text { scale down of the } \\
\text { sample and spike } \\
\text { additions } \\
\text { appropriately } \\
\text { (cut by } \\
\text { approximately } \\
\text { l/4) } \\
\end{array}$ & $\begin{array}{l}\text { Due to limited } \\
\text { amounts of } \\
\text { samples, waste } \\
\text { minimization and } \\
\text { ALARA concerns, } \\
\text { smaller amounts } \\
\text { of sample may } \\
\text { need to be used. }\end{array}$ \\
\hline $\begin{array}{l}\text { Section } 7.4: \text { Use } \\
300 \mathrm{~mL} \text { soivent }\end{array}$ & $\begin{array}{l}\text { Step 9.5: Use } 90 \\
\mathrm{~mL} \text { solvent. }\end{array}$ & $\begin{array}{l}\text { Deviation- scale } \\
\text { down of the } \\
\text { sample, solvent, } \\
\text { and spike } \\
\text { additions } \\
\text { appropriately } \\
\text { (cut by } \\
\text { approximately } \\
\text { l/4) } \\
\end{array}$ & $\begin{array}{l}\text { See above. This } \\
\text { al so reduces the } \\
\text { amount of methyi- } \\
\text { ene chloride } \\
\text { evaporated up the } \\
\text { hood. }\end{array}$ \\
\hline $\begin{array}{l}\text { Section 7.4: Use } \\
500 \mathrm{~mL} \text { round } \\
\text { flask. }\end{array}$ & $\begin{array}{l}\text { Step } 9.11 \text { : Use } \\
125 \mathrm{~mL} \text { round } \\
\text { flask with flat } \\
\text { bottom. }\end{array}$ & Deviation & $\begin{array}{l}\text { Smaller flasks } \\
\text { provide efficient } \\
\text { extraction in } \\
\text { limited hood } \\
\text { space. }\end{array}$ \\
\hline $\begin{array}{l}\text { Section } 7.10 \text { : } \\
\text { Add approximately } \\
50 \mathrm{~mL} \text { exchange } \\
\text { solvent once. }\end{array}$ & $\begin{array}{l}\text { Steps } 9.16 .1- \\
9.16 .2: \text { Add } 30 \\
\mathrm{~mL} \text { of hexane } \\
\text { three times }\end{array}$ & Deviation & $\begin{array}{l}\text { Repeating process } \\
\text { efficiently } \\
\text { removes first } \\
\text { solvent residue. }\end{array}$ \\
\hline $\begin{array}{l}\text { Section } 7.12 .2: \\
\text { Place the } \\
\text { concentrator tube } \\
\text { in a warm bath } \\
\left(35{ }^{\circ} \mathrm{C}\right) \text { and } \\
\text { evaporate to } 0.5 \\
\text { mL... }\end{array}$ & $\begin{array}{l}9.25 .1 \text { : Place } \\
\text { the tube in the } \\
\text { Reacti-vap } \\
\text { evaporator } \\
\text { assembly, with } \\
\text { the heating } \\
\text { module preheated } \\
\text { to } 40 \text { o C. } \\
\text { semivolatiles are } \\
\text { collected when } \\
\text { the MeCl level } \\
\text { reaches } 2 \mathrm{~mL} \text {. }\end{array}$ & Substitution & $\begin{array}{l}\text { Heat transfer is } \\
\text { done with a heat- } \\
\text { ing block instead } \\
\text { of a bath, } \\
\text { temperature } \\
\text { differences are } \\
\text { nominal. A final } \\
\text { volume of } 2 \mathrm{~mL} \\
\text { was necessary to } \\
\text { prevent losses. }\end{array}$ \\
\hline
\end{tabular}


HNF-SD-WM-DP-290, REV. 0

\section{APPENDIX D}

LA-523-136 
HNF-SD-WM-DP-290, REV. 0

THIS PAGE INTENTIONALLY LEFT BLANK 


\section{Appendix D \\ Comparison to $\mathrm{SW}-846^{8}$}

Method 8082, Revision 0, December 1996, Po7ychlorinated Biphenyls (PCBs) By Gas Chromatography

\begin{tabular}{|c|c|c|c|}
\hline SW-846 States: & Procedure States: & Type of change: & Reason for Change: \\
\hline $\begin{array}{l}\text { Table } 3 \text { or Step 2.5: (GC } \\
\text { operating Conditions or } \\
\text { summary) Injection } \\
\text { volume: } 2 \mu \text { (with a } \\
0.54 \mathrm{~mm} \text { id. megabore } \\
\text { column). or Step } 7.4 .5 \\
\text { A } 2-\mu \mathrm{L} \text { injection of each } \\
\text { calibration standard is } \\
\text { recommended. Other } \\
\text { injection volumes may be } \\
\text { used if the analyst can } \\
\text { demonstrate adequate } \\
\text { sensitivity for the } \\
\text { compounds. }\end{array}$ & $\begin{array}{l}\text { Step 10.1: Gas } \\
\text { Chromatograph Operating } \\
\text { Conditions - Injection } \\
\text { Volume: } 1 \text { HL }\end{array}$ & Substitution & $\begin{array}{l}\text { Smaller injection } \\
\text { needed for mid-bore } \\
\text { colum }(0.32 \mathrm{~mm} \text { id). } \\
\text { otherwise injection of } \\
\text { oil sample extracts } \\
\text { will compromise the } \\
\text { system. The "Suggested" } \\
\text { cal ibration levels of } \\
100,150,200,400 \text {, and } \\
600 \text { ng/mL extends above } \\
\text { the linear cal ibration } \\
\text { range for a } 1 \mu \mathrm{L} \\
\text { injection. Hence the } \\
\text { calibration levels } \\
\text { selected are at } 20,50 \text {, } \\
100,200, \text { and } 400 \\
\text { ng/mL. }\end{array}$ \\
\hline $\begin{array}{l}\text { Step 5.5: Stock } \\
\text { standard solutions }(1000 \\
\mathrm{mg} / \mathrm{L})\end{array}$ & $\begin{array}{l}\text { Step } 4.1 .2: \text {...the QC } \\
\text { reference sample } \\
\text { concentrate should } \\
\text { contain the most repre- } \\
\text { sentative multicomponent } \\
\text { parameter at a concen- } \\
\text { tration of } 50 \mathrm{mg} / \mathrm{L} \text {. (In } \\
\text { fact, } 40 \mathrm{mg} / \mathrm{L} \text { Standards } \\
\text { custom made by Restek } \\
\text { are used so that the } \\
\text { threshold limit of } 50 \\
\text { PPM will not be met or } \\
\text { exceeded.) }\end{array}$ & $\begin{array}{l}\text { Deviation- scale down of } \\
\text { the spike additions } \\
\text { appropriately (cut by } \\
\text { approximately } i / 100 \text { ). }\end{array}$ & $\begin{array}{l}\text { ISCA Regulation } \\
\text { concerns, waste } \\
\text { minimization, otherwise } \\
\text { all spiked matrices } \\
\text { must be isolated and } \\
\text { treated as mixed } \\
\text { regulated waste without } \\
\text { a disposal path. Also, } \\
\text { the level of QC spike } \\
\text { suggested is above the } \\
\text { calibration levels of } \\
\text { the GC. }\end{array}$ \\
\hline $\begin{array}{l}\text { Section } 8.3 .1: \text { A } 1 \mathrm{~mL} \\
\text { volume of the (QC) } \\
\text { concentrate spiked into } \\
1 \mathrm{~L} \text { of organic free } \\
\text { water will result in a } \\
\text { sample concentration of } \\
10-50 \mathrm{\mu g} / \mathrm{L} \text {. }\end{array}$ & $\begin{array}{l}\text { See step } 9.1 .5 .2 \text { of } \\
\text { LA-523-115: If not, add } \\
\text { the water diluent first, } \\
\text { so the sample plus } \\
\text { diluent equals } 100 \mathrm{~mL} \text {. }\end{array}$ & Deviation & $\begin{array}{l}\text { Custom semi-micro } \\
\text { liquid-liquid extractor } \\
\text { used in LA-523-115 } \\
\text { requires less volume } \\
\text { (ca. } 100 \mathrm{~mL} \text { ) ALARA } \\
\text { concerns and limited } \\
\text { sample and hood size } \\
\text { make snaller extraction } \\
\text { vessel a necessity. }\end{array}$ \\
\hline $\begin{array}{l}8081 \mathrm{~A} \text { and } 8082 \text { are } \\
\text { separate methods. }\end{array}$ & $\begin{array}{l}8081 \mathrm{~A} \text { and } 8082 \text { have been } \\
\text { combined. }\end{array}$ & Two methods combined. & $\begin{array}{l}\text { Sulfuric acid cleanup } \\
\text { limits pesticide } \\
\text { applicability. Acid } \\
\text { cleanup rarely used for } \\
\text { pesticides. }\end{array}$ \\
\hline
\end{tabular}

${ }^{8}$ NOTE: See LA-523-115 and LA-523-138 for sample preparation comparisons. 
HNF-SD-HM-DP-290, REV. 0

REQUEST FOR SPECIAL ANALYSIS

131 
HNF-SD-HM-DP-290, REV. 0

THIS PAGE INTENTIONALLY LEFT BLANK 
$\because 101$

$12 / 30 / 97$

PAUL KEMPF 3. Submitted By

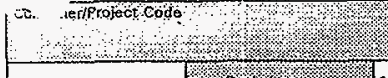

9.0 .0 .0 .83 \begin{tabular}{l|l} 
6. TPCN & 7. Org. Code
\end{tabular}

3. Customer IO No.

PALL KEMPF

5. Requestor's Phone/MSIN/FAX

373-2435 T6-20

$373-3176$

TANK 101

mHK-101 $\mathrm{FC}$.

\begin{tabular}{|l|l|}
\hline $\begin{array}{c}\text { 10. Volumo } \\
\text { of Samplo }\end{array}$ & \\
\hline 11 & \\
\hline & \\
\hline
\end{tabular}

11. Matrix of Samplo

12. Requested Analyses

13. Expoctod Rango

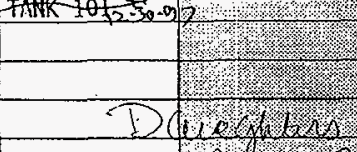

syernate

SOL Lupuid

14. Does samplo have a MSDS?

$\square$ Yes HEHF assigned MSDS No.

[X] No Dezcription of procoss that produced wasto/eample:

Stean fondersate for the_222S_Laboratory skW $12 / 31 / 97$

F.

this sample RCRA listed? $\square$ Yeo $[X$ No

Applicable Listed Waste Cọdes:

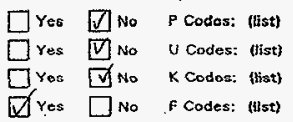

Applicable Charaicteristic Codes:

CB: Doos this wasto/sampla contain PCBs?
$\square$ Yes Over $500 \mathrm{ppm}$
If YES, what is the-source of the PCBs?
$\square$ Ves Over $50 \mathrm{ppm}$
$\square$ Transformer, capacitor, or ballast
$\square$ Ves pCBs are suspected
$[\mathrm{X}]$ No PCBs are kuspected
$\square$ other. specify
unknown

16. Sample Disposition

$\square$ Retum to Customer

$\square$ somples found to contain PCBs will be returned to the customer

[X] Dlspose of per tacility procedures with applicd chargos for analyses and disposal

17. OC Riequired [XPor 222-S Laboratory Quality Assuranco Plan (HNF-SD-CP-QAPP-O16)

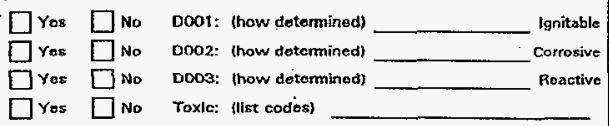

$\square$ other (list referenco document or attach)

18. Specizl Instructions ispecial Storage Requiraments. Roporting format, hoblding timpos, otc.)

NONE

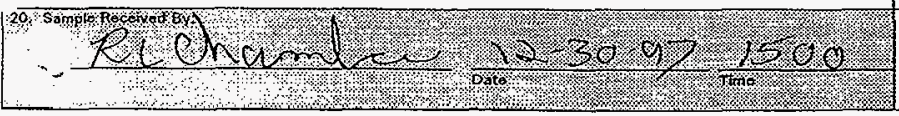

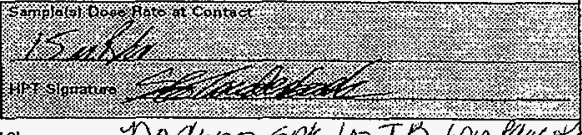

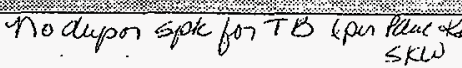
प2 Weeks $\square 4$ Weoks
国] othor ASAP

21. Chain of Custody

QD No $\square$ Yor

Number: 
HNF-SD-WM-DP-290, REV. 0

CLP-LIKE FORMS SUMMARY RESULT FORMS 
HNF-SD-WM-DP-290, REV. 0

THIS PAGE INTENTIONALLY LEFT BLANK 
Data File: /chem/gc2.i/012398.b/023f0201.d

\section{2-s Laboratory \\ TARGET COMPOUNDS}

Client Name:

Lab Smp Id: S97S00235

Sample Location:

Sample Date:

Sample Matrix: SOII

Analysis Type: PEST

Data Type: GC MULTI COMP

Misc Info: 975023500927
Client SDG: 012398

Client Smp ID: S97S00235

Sample Point:

Date Received:

Quant Type: ESTD

Level: LoW

operator: Gerald Ross

CONCENTRATION UNITS:

\begin{tabular}{|c|c|c|}
\hline $\begin{array}{l}12674-11-2----- \text { Aroclor }-1016 \\
11104-28-2----- \text { Aroclor }-1221 \\
1114-16-5------ \text { Aroclor }-1232 \\
53469-21-9----- \text { Aroclor }-1242 \\
12672-29-6----- \text { Aroclor }-1248 \\
11097-69-1------ \text { Aroclor }-1254 \\
11096-82-5----- \text { Aroclor }-1260\end{array}$ & $\begin{array}{l}1485.8 \\
670.00 \\
330.00 \\
330.00 \\
6204.1 \\
5460.9 \\
1706.4\end{array}$ & $\begin{array}{c}\mathrm{U} \\
\mathbf{U} \\
\mathbf{U}\end{array}$ \\
\hline 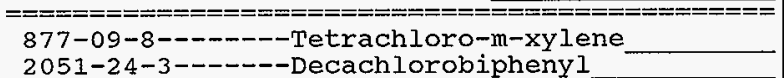 & $\begin{aligned}===== & ==== \\
& 114.74 \\
& 94.860\end{aligned}$ & $\Rightarrow==$ \\
\hline
\end{tabular}


Data File: /chem/gc2.i/012398.b/025f0201.d

\section{2-S Laboratory}

\section{TARGET COMPOUNDS}

Client Name:

Lab Smp Id: 97S 0235D00929

Sample Location:

Sample Date:

Sample Matrix: SOIL

Analysis Type: PEST

Data Type: GC MULTI COMP

Misc Info: 97S 0235D00929
Client SDG: 012398

Client Smp ID: 97S 0235D00929

Sample Point:

Date Received:

Quant TYpe: ESTD

Level: LOW

Operator: Gerald Ross

CONCENTRATION UNITS :

( $\mathrm{ug} / \mathrm{L}$ or $\mathrm{ug} / \mathrm{KG}$ ) ug/Kg

\begin{tabular}{|c|}
\hline $\begin{array}{l}12674-11-2-----A r o c l o r-1016 \\
11104-28-2-----A r o c l o r-1221 \\
1114-16-5----- \text { Aroclor }-1232 \\
53469-21-9----- \text { Aroclor }-1242 \\
12672-29-6----- \text { Aroclor }-1248 \\
11097-69-1----- \text { Aroclor }-1254 \\
11096-82-5----- \text { Aroclor }-1260\end{array}$ \\
\hline 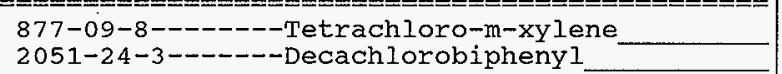 \\
\hline
\end{tabular}

\begin{tabular}{|r|r|}
1881.7 & \\
670.00 & $\mathrm{U}$ \\
330.00 & $\mathrm{U}$ \\
330.00 & $\mathrm{U}$ \\
6921.1 & \\
5873.3 & \\
1803.7 & \\
133.93 & \\
73.325 &
\end{tabular}




\section{2-S Laboratory \\ TARGET COMPOUNDS}

Client Name:

Lab Smp Id: s97S00235MS

Sample Location:

Sample Date:

Sample Matrix: SoIL

Analysis Type: PEST

Data Type: GC MULTI COMP

Misc Info: $97 \mathrm{~S}$ 0235MS00931
Client SDG: 012398

client Smp ID: S97S00235MS

Sample Point:

Date Received:

Quant Type: ESTD

Level: LOW

Operator: Gerald Ross

CONCENTRATION UNITS :

( $u g / L$ or $u g / K G$ ) ug/Kg

\begin{tabular}{|c|c|c|}
\hline $\begin{array}{l}12674-11-2----- \text { Aroclor }-1016 \\
11104-28-2----- \text { Aroclor }-1221 \\
1114-16-5------ \text { Aroclor }-1232 \\
53469-21-9---- \text { Aroclor }-1242 \\
12672-29-6----- \text { Aroclor }-1248 \\
11097-69-1------ \text { Aroclor }-1254 \\
11096-82-5----- \text { Aroclor }-1260\end{array}$ & $\begin{array}{l}1139.9 \\
670.00 \\
330.00 \\
330.00 \\
8129.6 \\
6472.1 \\
2488.0\end{array}$ & $\begin{array}{l}\mathrm{U} \\
\mathrm{U} \\
\mathrm{U}\end{array}$ \\
\hline 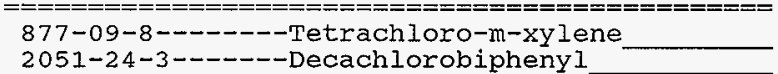 & 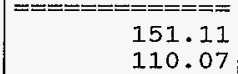 & $m===$ \\
\hline
\end{tabular}


Data File: /chem/gc2.i/012398.b/027f0201.d

\section{2-S Laboratory \\ TARGET COMPOUNDS}

Client Name:

Lab Smp Id: s97500235MSD

Sample Location:

Sample Date:

Sample Matrix: SoIL

Analysis Type: PEST

Data Type: GC MULTI COMP

Misc Info: 97S 0235MSD00933 client SDG: 012398

Client Smp ID: S97500235MSD

Sample Point:

Date Received:

Quant Type: ESTD

Level: LOW

Operator: Gerald Ross

CONCENTRATION UNITS:

(ug/L or ug/KG) ug/Kg

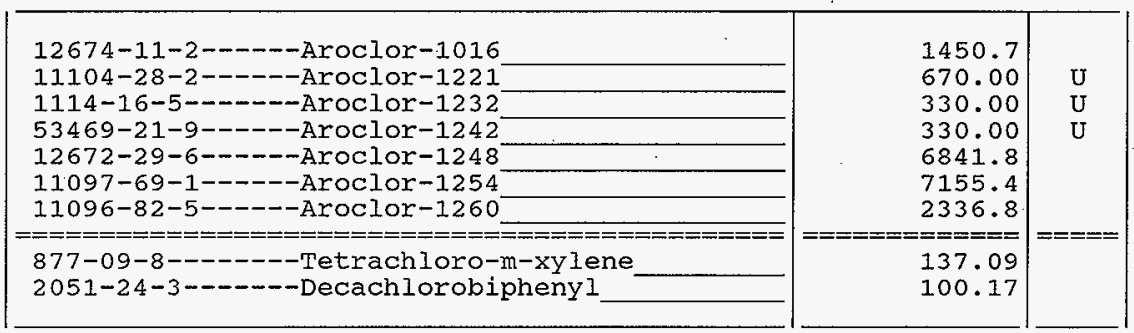




\section{HNF-SD-WM-DP-290 REV. 0}

Data File: /chem/gc2.i/012398.b/021f0201.d

\section{2-S Laboratory}

TARGET COMPOUNDS

Client Name:

Lab Smp Id: meth blnk 00935 Sample Location:

Sample Date:

Sample Matrix: SoIL

Analysis Type: PEST

Data Type: GC MULTI COMP

Misc Info: meth blnk 00935 client SDG: 012398

Client Smp ID: meth blnk 00935

Sample point:

Date Received:

Quant Type: ESTD

Level: LOW

Operator: Gerald Ross

CONCENTRATION UNITS:

CAS NO.

COMPOUND

( $\mathrm{ug} / \mathrm{L}$ or $\mathrm{ug} / \mathrm{KG}$ ) ug/Kg

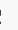

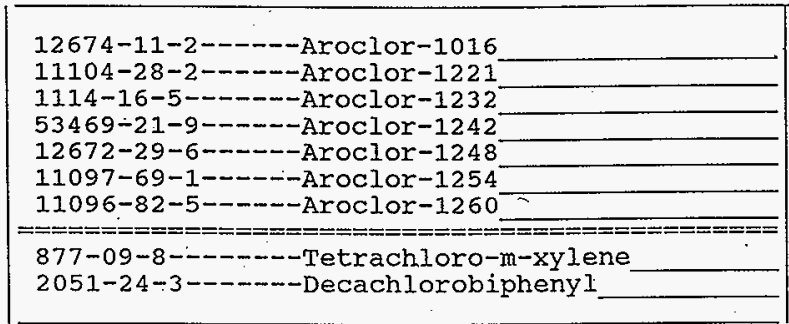

\begin{tabular}{|c|c|}
33.000 & $\mathrm{U}$ \\
67.000 & $\mathrm{U}$ \\
33.000 & $\mathrm{U}$ \\
33.000 & $\mathrm{U}$ \\
33.000 & $\mathrm{U}$ \\
3.7100 & $\mathrm{U}$ \\
33.000 & $\mathrm{U}$ \\
\hline 1.590 \\
107.92
\end{tabular}




\section{2-S Laboratory \\ TARGET COMPOUNDS}

client Name:

Lab Smp Id: LCS spk 00937

Sample Location:

Sample Date:

Sample Matrix: SOIL

Analysis Type: PEST

Data Type: . GC MULTI COMP

Misc Info: 000000937
Client SDG: 012398

Client Smp ID: LCS spk 00937

Sample point:

Date Received:

Quant Type: ESTD

Level: LOW

Operator: Gerald Ross

CONCENTRATION UNITS:

(ug/L or $u g / K G$ ) ug/Kg

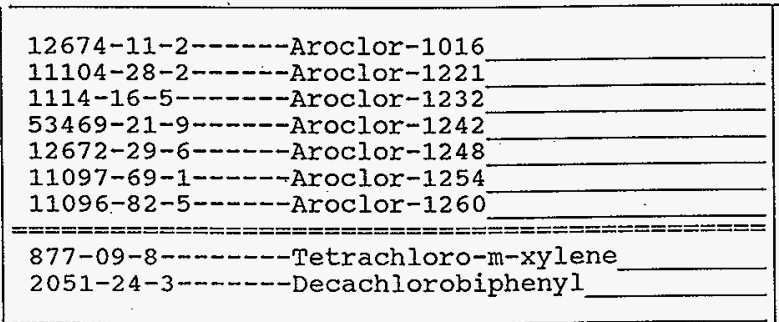

\begin{tabular}{rr|}
92.902 & \\
67.000 & $\mathrm{U}$ \\
33.000 & $\mathrm{U}$ \\
33.000 & $\mathrm{U}$ \\
33.000 & $\mathrm{U}$ \\
3.7100 & $\mathrm{U}$ \\
91.835 & \\
\hline 12.934 & \\
69.212 & $====$
\end{tabular}


HNF-SD-WM-DP-290, REV. 0

EXTRACTION BENCHSHEETS 
HNF-SD-WM-DP-290, REV. 0

THIS PAGE INTENTIONALLY LEFT BLANK 


\begin{tabular}{|c|c|}
\hline FILE LOCATION : & $\begin{array}{l}\text { EXTRACTION } \angle A-523-138 \\
\text { PROCEDURE: } \angle A-52\end{array}$ \\
\hline $\begin{array}{l}5975000235 \text { HOINTK } \\
\text { WORK PKG./R NUMBER }\end{array}$ & EXTRACTION ANALYS \\
\hline MATRIX: ALCC/HEXANE & DATE/STSTEM: $1-8.98$ \\
\hline
\end{tabular}

ANALYSIS

PROCEDURE:

INSTRUMENTAL ANALYST:

\begin{tabular}{|c|c|c|c|c|c|}
\hline $\begin{array}{l}\text { SAMPLE } \\
\text { NUMBER }\end{array}$ & $\begin{array}{l}\text { HEIGHT } \\
\text { VOLUHE }\end{array}$ & $\begin{array}{l}\text { BAR } \\
\text { CODE }\end{array}$ & ; & $\begin{array}{l}\text { FIWAL } \\
\text { SOLYENT }\end{array}$ & $\begin{array}{l}\text { FINAL: } \\
\text { VOLUME }\end{array}$ \\
\hline
\end{tabular}

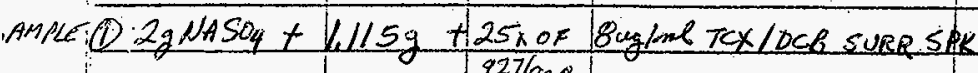
$927 / 928$

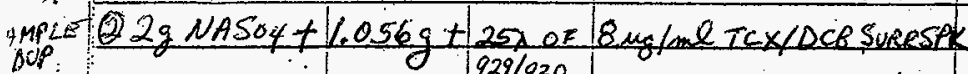
Hexaver $2 \mathrm{me}$

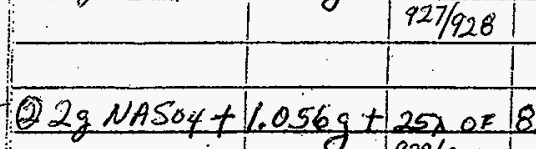
$\div$

1

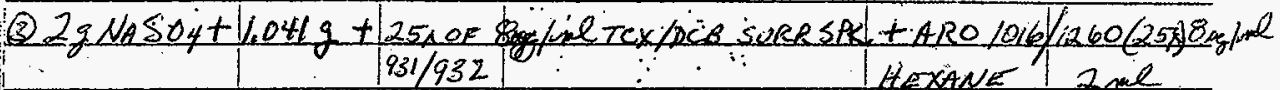

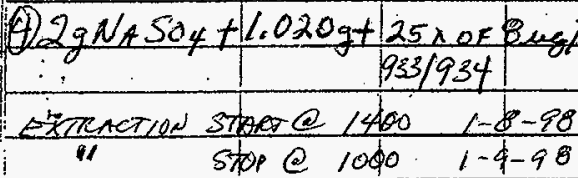

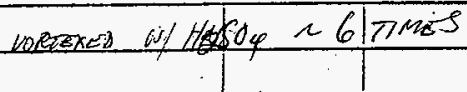

\begin{tabular}{|c|c|c|c|c|c|c|c|}
\hline $\begin{array}{l}\text { SURR. } \\
\text { NAME }\end{array}$ & $\begin{array}{l}\text { VOLUME } \\
\text { (UL) }\end{array}$ & ID & CONC. & $\begin{array}{l}\text { MATRIX } \\
\text { SPIXE } \\
\text { MAAAE }\end{array}$ & $\begin{array}{l}\text { YOLUNE } \\
(U L)\end{array}$ & $\begin{array}{l}10^{\circ} \\
\text { NUMBER }\end{array}$ & conc. \\
\hline$\pi C \times / D C B$ & $25 \lambda$ & & $8 \mathrm{mo} / \mathrm{mel}$ & ARO 1016 & $12360 \cdot 25 \lambda$ & & Explal \\
\hline & & & & & & & \\
\hline & & & & & & & \\
\hline
\end{tabular}

\begin{tabular}{|l|l|l|l|l|l|}
\hline $\begin{array}{l}\text { AMALYSIS } \\
\text { YOLUME } \\
\text { OF EXTRACT } \\
\text { USED }\end{array}$ & $\begin{array}{l}\text { INTERMAL } \\
\text { STANDARO } \\
\text { SUPPLIER }\end{array}$ & $\begin{array}{l}\text { VOLUME } \\
\text { AODED } \\
\text { (UL) }\end{array}$ & $\begin{array}{l}\text { I0 MUMBER } \\
\text { LOT MUABER }\end{array}$ & $\begin{array}{l}\text { CONC. } \\
\text { INITIAL }\end{array}$ & $\begin{array}{l}\text { CONC. } \\
\text { FIMAL }\end{array}$ \\
\hline & & & & & \\
\hline
\end{tabular}


HNF-SD-WM-DP-290, REV. 0

GC SEQUENCE 
HNF-SD-WM-DP-290, REV. 0

THIS PAGE INTENTIONALLY LEFT BLANK

24 


\section{Sequence Table}

\begin{tabular}{|c|c|c|c|c|c|c|}
\hline \multirow{2}{*}{$\begin{array}{l}\text { Inj. } \\
\text { FRONT }\end{array}$} & $\begin{array}{l}\text { Seq. } \\
\text { Line }\end{array}$ & $\begin{array}{l}\text { Cal. } \\
\text { Line }\end{array}$ & $\begin{array}{l}\text { Method } \\
\text { Name }\end{array}$ & $\begin{array}{l}\text { From } \\
\text { vial }\end{array}$ & $\begin{array}{c}\text { To } \\
\text { vial }\end{array}$ & $\begin{array}{l}\text { Inj/ } \\
\text { Vial }\end{array}$ \\
\hline & $\frac{1}{2}$ & & $\begin{array}{l}\text { PCB-FRNT } \\
\text { PCB-FRNT }\end{array}$ & $\frac{1}{3}$ & $\begin{array}{r}2 \\
28\end{array}$ & $\begin{array}{l}3 \\
1\end{array}$ \\
\hline & $\begin{array}{l}1 \\
2\end{array}$ & & $\begin{array}{l}\text { PCB-REAR } \\
\text { PCB-REAR }\end{array}$ & $\begin{array}{l}51 \\
53\end{array}$ & $\begin{array}{l}52 \\
78\end{array}$ & $\begin{array}{l}3 \\
1\end{array}$ \\
\hline
\end{tabular}

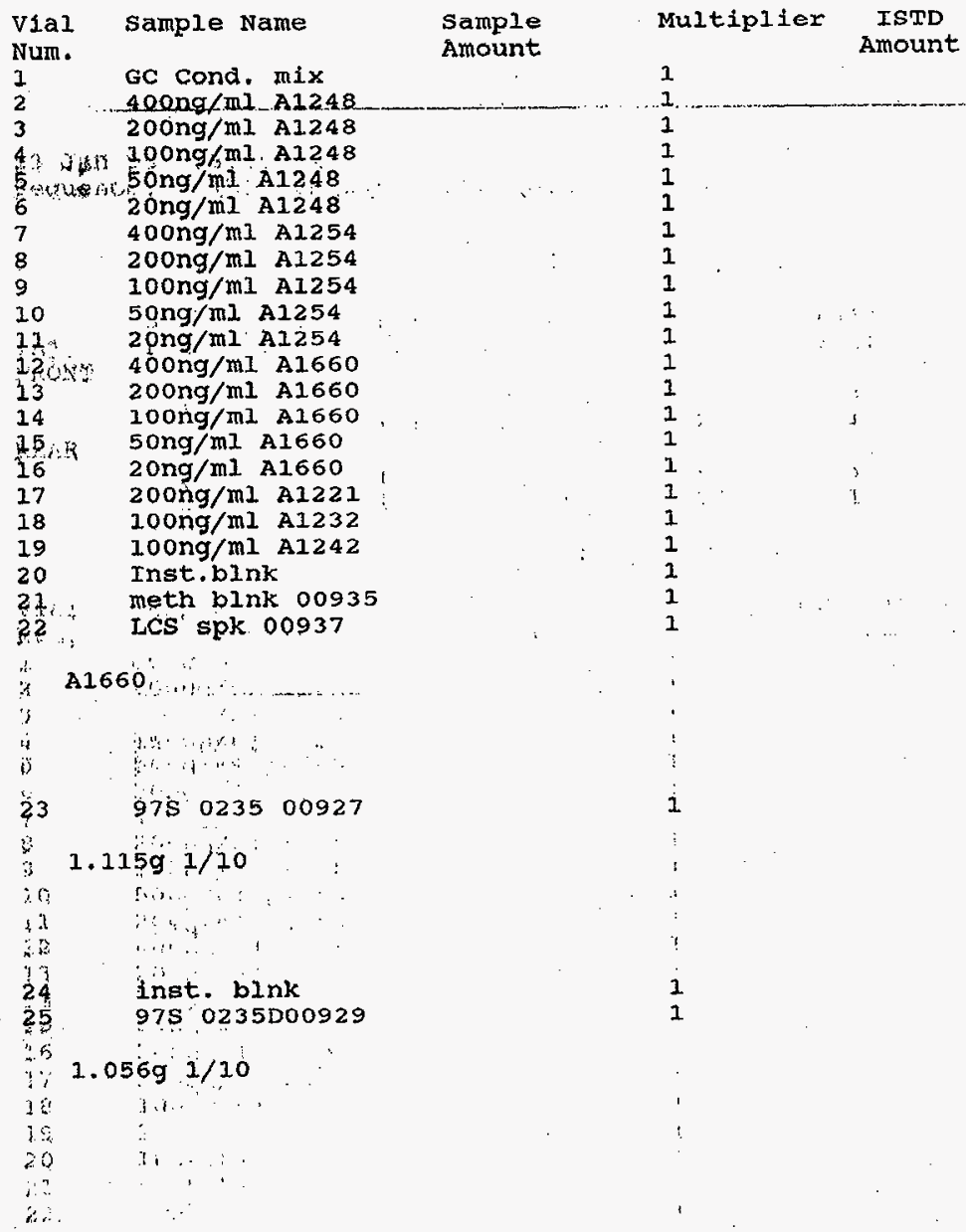




\section{HNF-SD-WMADP-290 REV. 0}

$23 \operatorname{Jan} 98 \quad 08: 45 \quad \mathrm{AM}$

page 2

Sequence: C: \HPCHEM \2\SEQUENCE\012398A. SEQ

Vial sample Name

Num.

26

$9750235 \mathrm{Ms} 00931$

$1.04 \mathrm{ig} \cdot 1 / 10$

27

$978 \quad 0235 \mathrm{MSD} 00933$

Sample

Amount

Sample Table

1

$1.02091 / 10$

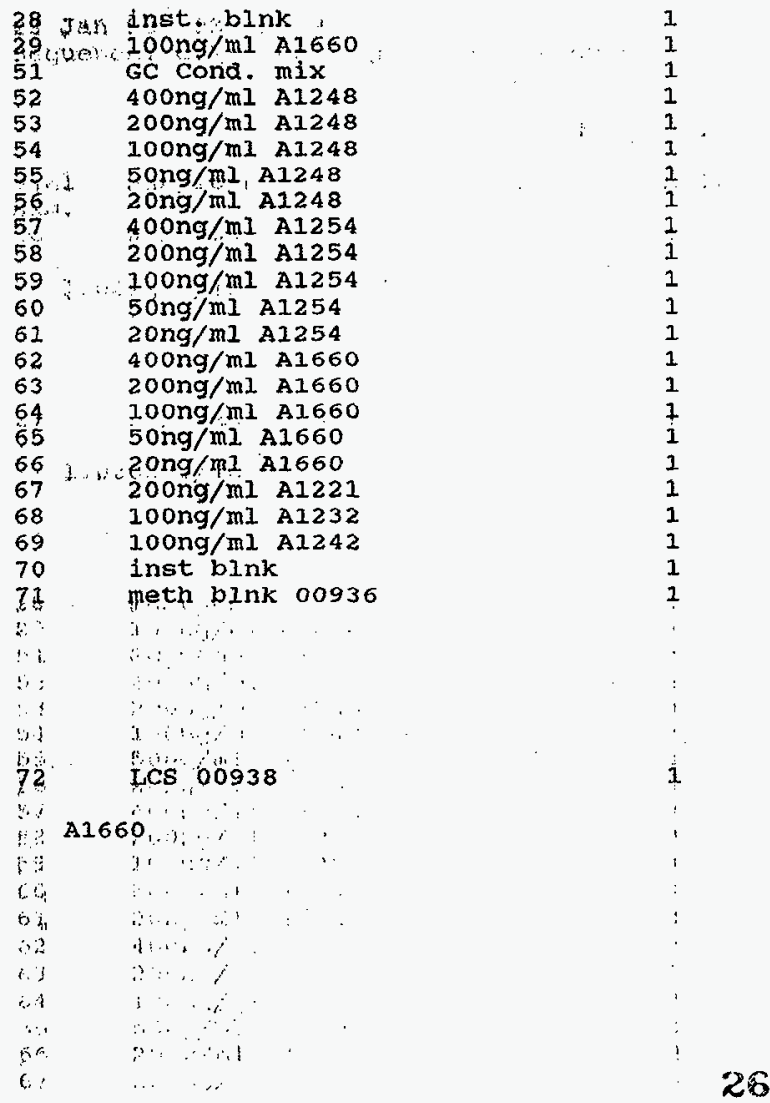


$23 \operatorname{Jan} 98 \quad 08: 45 \mathrm{AM}$

HNF-SD-WM-DP-290 REV. 0

Sequence: C: \HPCHEM \2\SEQUENCE\012398A.SEQ
Sample Table
Vial Sample Name
Num.
$\because: ?$,... Name
73
975023500928
Sample Amount

Multiplier $\underset{\text { Amount }}{\text { ISTD }}$
1

page 3

1.1159

74 , inst bink

1

$1.056 \mathrm{~g}$

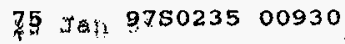

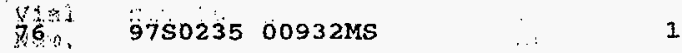

$1.04 \mathrm{1g}$

$\therefore$. ㄴ.?

\section{7}

74

$1.020 \mathrm{~g}$

975023500934 MSD

$\therefore \cdots$

\section{8 inst. blink}

79 1,00ng/mL A1660

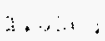

is

?; (3.: : ;
1

1

1 
HNF-SD-WM-DP-290, REV. 0

INITIAL CALIBRATION REPORT 
HNF-SD-WM-DP-290, REV. 0

THIS PAGE INTENTIONALLY LEFT BLANK

\section{9}




\section{2-S Laboratory}

INITIAL CALIBRATION DATA

Start Cal Date : 06-AUG-97 17:19

End Cal Date : 24-JAN-1998 05:06

Quant Method : ESTD

Origin : Force

Target Version : 3.10

Integrator : HP Genie

Method file :/chem/gc2.i/012398.b/rcrapcb.m

Cal Date

Curve Type

: 27-Jan-1998 07:17 gar

: Average

Calibration File Names:

Level 1: /chem/gc2.i/012398.b/016f0201.d

Level 2: /chem/gc2.i/012398.b/015f0201.d

Level 3:/chem/gc2.i/012398.b/014f0201.d

Level 4:/chem/gc2.i/012398.b/013f0201.d

Level 5: /chem/gc2.i/012398.b/012f0201.d

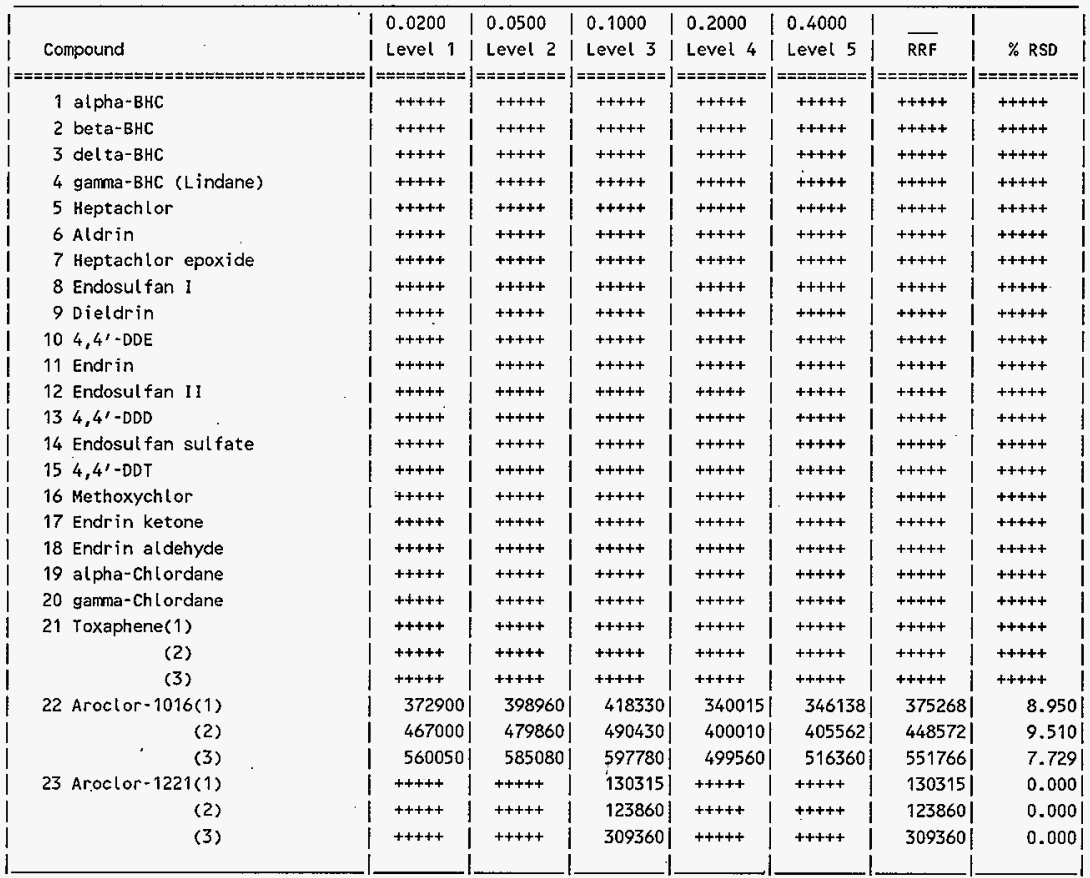




\section{HNF-SD-WM-DP-290 REY. 0}

Report Date : 27-Jan-1998 07:17

Page 2

\section{2-S Laboratory}

INITIAL CALIBRATION DATA

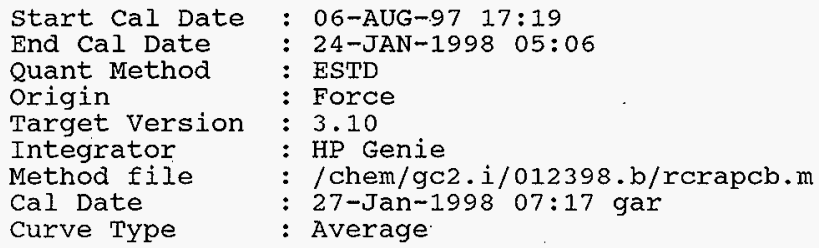

\begin{tabular}{|c|c|c|c|c|c|c|c|c|}
\hline & & 0.0200 & 0.0500 & 0.1000 & $|0.2000|$ & $0.4000 \mid$ & & | \\
\hline & Compound & Level 1 & Level 2 & Level 3 & Level 4 & Level 5 & $\overline{R R F}$ & $\%$ RSD \\
\hline \multicolumn{9}{|c|}{ 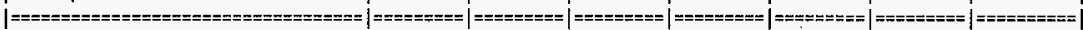 } \\
\hline & 24 Aroclor $-1232(1)$ & +++++ & ++++ & 9870 & ++++ & +++++ & $9870 i$ & 0.000 \\
\hline & (2) & ++++ & +++++ & 12810 & +++++ & ++++ & $12810 \mid$ & 0.000 \\
\hline & (3) & +++++ & ++++ & 144601 & +++++ & +++++ & 144601 & 0.0001 \\
\hline & 25 Aroclor $-1242(1)$ & +++++ & +++++ & 27310 & +++++ & +++++ & 27310 & 0.000 \\
\hline & (2) & $t+t+t$ & ++++ & 604201 & ++++ & ++++ & 60420 & 0.0001 \\
\hline & (3) & ++++4 & $+t+t+$ & $30780 \mid$ & +++++ & +++++ & $30780 \mid$ & 0.000 \\
\hline & 26 Aroclor $-1248(1)$ & 401750 & 399020 & $361130 \mid$ & 366260 & 3353301 & 372698 & 7.4801 \\
\hline & (2) & 458700 & 493740 & 416530 & 414865 & 395092 & 435786 & 9.142 \\
\hline & (3) & 5620001 & 555060 & 462270 & 459740 & 4383901 & 4954921 & 11.774 \\
\hline & 27 Aroclor $-1254(1)$ & 4980501 & 3741601 & +++++ & 388470 & 494245 & 4387311 & 15.174 \\
\hline & (2) & 6699501 & 4941401 & +++++ & 515700 & 6433401 & 580782 & 15.274 \\
\hline & (3) & 613850 & 433880 & $++++\quad i$ & 452665 & $601848 \mid$ & $525561 \mid$ & 18.162 \\
\hline & 28 Aroclor $-1260(1)$ & $616100 \mid$ & 586620 & 569840 이 & 4942401 & 455742 & 544508 & 12.3011 \\
\hline & (2) & 621500 & 595100 & 567060 & 503415 & 449508 & 547316 & 12.813 \\
\hline & (3) & 5896000 & 502480 & 523760 & 432700 & $385842 \mid$ & 486876 & $16.334 \mid$ \\
\hline \multicolumn{9}{|c|}{ 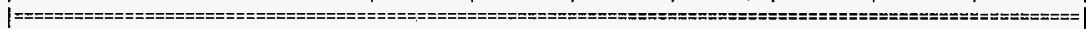 } \\
\hline$\$$ & 29 Tetrachloro-m-xylene & $9447800 \mid$ & $10393400 \mid$ & $10907440 \mid$ & $8788180 \mid$ & 8755855 & $9658535 \mid$ & $9.980 \mid$ \\
\hline$\$$ & 30 Decachlorobiphenyl & ++++ & $\dot{5} 849320$ & 4463060 & 4553640 & +++++ & $4955340 \mid$ & 15.650 \\
\hline
\end{tabular}


HNF-SD-WM-DP-290, REV. 0

RAW DATA

32 
HNF-SD-WMY-DP-290, REV. 0

THIS PAGE INTENTIONALLY LEFT BLANK 
Data File: /chem/gc2.i/012398.b/002f0103.d

Page 1

Report Date: 26-Jan-1998 15:30

\title{
HNF-SD-WNA-DP-290 REV. 0
}

\author{
222-S Laboratory
}

Data file: /chem/gc2.i/012398.b/002f0103.d

Lab Smp Id: 400ng/ml A1248

Inj Date : 23-JAN-1998 14:30

Operator : Gerald Ross

Smp Info : $400 \mathrm{ng} / \mathrm{ml} \mathrm{A1248}$

Misc Info : $400 \mathrm{ng} / \mathrm{ml} \mathrm{A} 1248$

Comment

Method

Meth Date : 26-Jan-1998 15:30 gar

Cal Date : 23-JAN-1998 14:30

Als bottle: 1

Dil Factor: 1.000

Integrator: HP Genie

Target Version: 3.10
Client Smp ID: $400 \mathrm{ng} / \mathrm{ml}$ A1248

Inst ID: $g c 2 . i$

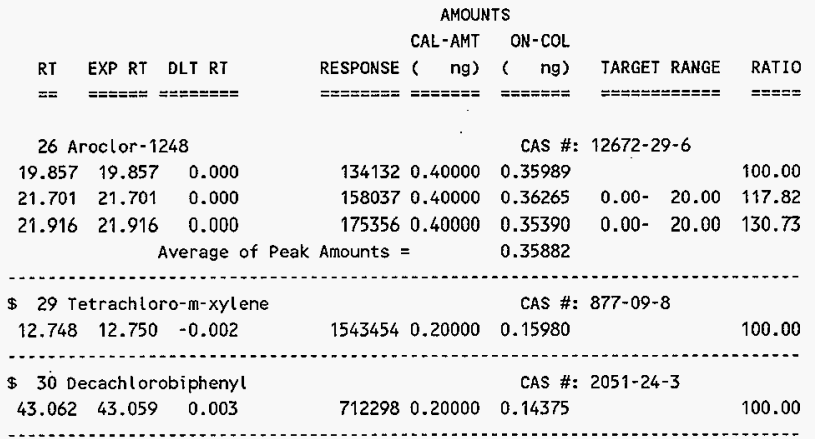




\section{HNF-SD-WM-DP-290 REV. 0}

Data File: /chem/gc2.i/012398.b/003f0201.d

Report Date: 26-Jan-1998 15:30

Data file : /chem/gc2,i/012398.b/003f0201.d

Lab Smp Id: $200 \mathrm{ng} / \mathrm{ml}$ A1248

Inj Date : 23-JAN-98 15:32

Operator : Gerald Ross

Smp Info : $200 \mathrm{ng} / \mathrm{ml}$ A1248

Misc Info: $200 \mathrm{ng} / \mathrm{ml} \mathrm{A1248}$

Comment

Method : /chem/gc2.i/012398.b/rcrapcb.m

Meth Date : 26-Jan-1998 15:30 gar

Cal Date : 23-JAN-98 15:32

Als bottle: 1

Dil Factor: 1.000

Integrator: HP Genie

Target Version: 3.10

Client Smp ID: $200 \mathrm{ng} / \mathrm{ml}$ A1248

Inst ID: $g c 2 . i$

Quant Type: ESTD

Cal File: $003 f 0201 . d$

Calibration Sample, Level: 4

Compound Sublist: AR1248.sub

Sample Matrix: WATER

\begin{tabular}{|c|c|c|c|c|c|c|c|}
\hline & & & & AMOUN & & & \\
\hline & & & & CAL-AMT & $\mathrm{ON}-\mathrm{COL}$ & & \\
\hline $\mathrm{RT}$ & EXP RT & $L T R T$ & RESPONSE & ( $n g)$ & ( $n g$ ) & TARGET RANGE & RATIO \\
\hline$=$ & $==x==0$ & $======$ & 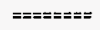 & 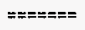 & $== \pm== \pm$ & 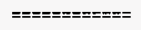 & $=====$ \\
\hline $26 \mathrm{Ar}$ & oclor-12 & & & & CAS \#: & $12672-29-6$ & \\
\hline 19.857 & 19.857 & 0.000 & 73252 & 0.20000 & 0.19654 & & 100.00 \\
\hline 21.701 & 21.701 & 0.000 & 82973 & 0.20000 & 0.19040 & $0.00-20.00$ & 113.27 \\
\hline 21.917 & 21.917 & 0.000 & 91948 & $0.20000^{\circ}$ & 0.18557 & $0.00-20.00$ & 125.52 \\
\hline & & lerage 0 & Amounts = & & 0.19084 & & \\
\hline$\$ 29 \mathrm{re}$ & trachlor & $-m-x y l e$ & & & CAS \#: & $877-09-8$ & \\
\hline 12.748 & 12.750 & -0.002 & 943008 & 0.10000 & 0 & & 100.00 \\
\hline$\$ 30 \mathrm{De}$ & ecac & iph & & & CAS \#: & $2051-24-3$ & \\
\hline 43.063 & 43.059 & 0.004 & 317122 & 0.10000 & 0 & & 100.00 \\
\hline
\end{tabular}




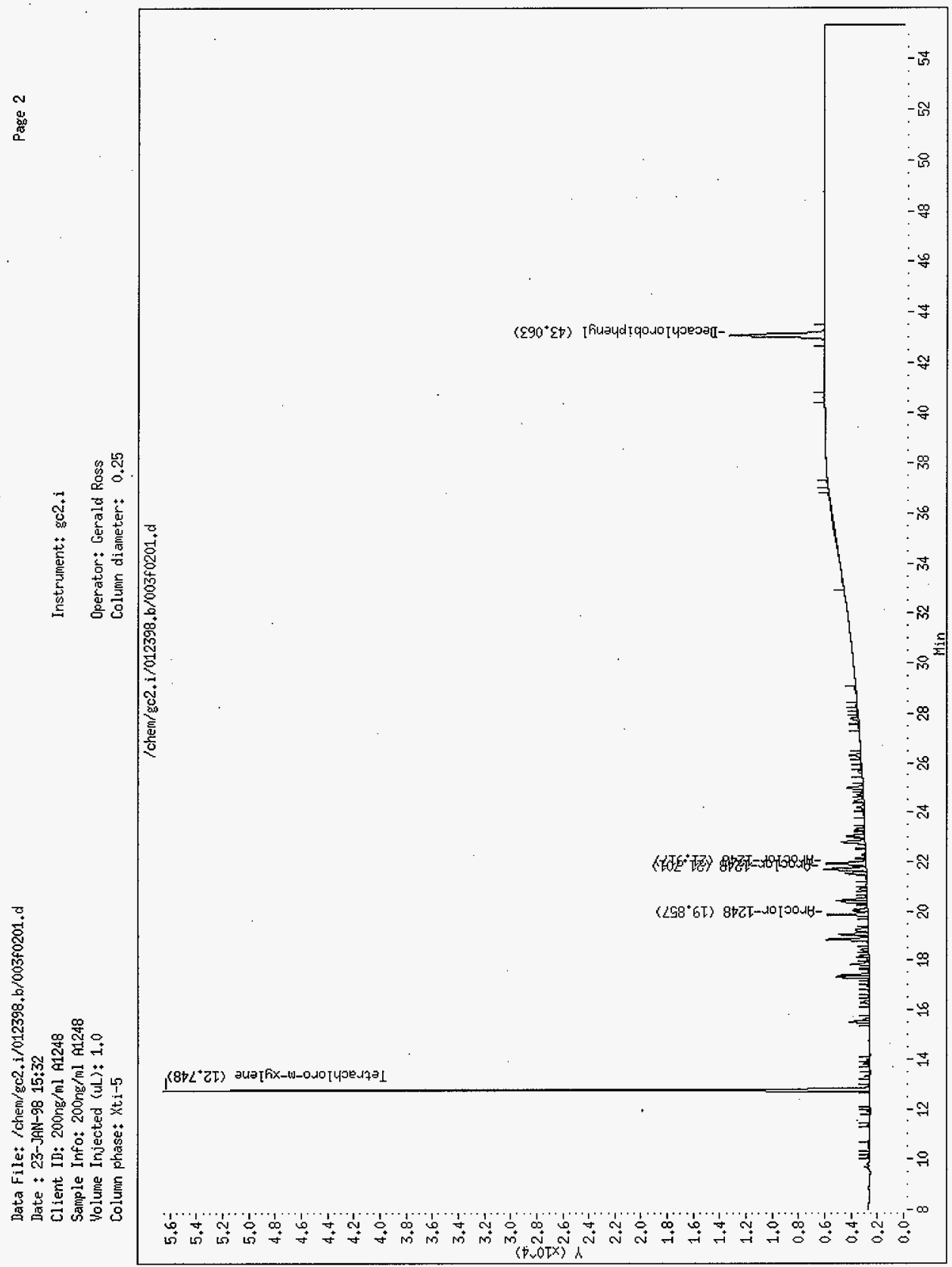


Data File: /chem/gc2.i/012398.b/004f0201.d

Report Date: 26-Jan-1998 15:30

\section{2-S Laboratory}

Data file:/chem/gc2.i/012398.b/004f0201.d

Lab Smp Id: 100ng/ml A1248

Inj Date : 23-JAN-98 16:35

Operator : Gerald Ross

Smp Info : $100 \mathrm{ng} / \mathrm{ml} \mathrm{A1248}$

Misc Info: $100 \mathrm{ng} / \mathrm{ml}$ A1248

comment

Method : /chem/gc2.i/012398.b/rcrapcb.m

Meth Date : 26-Jan-1998 15:30 gar

Cal Date : 23-JAN-98 16:35

Als bottle: 1

Dil Factor: 1.000

Integrator: HP. Genie

Target Version: 3.10
Client Smp ID: 100ng/ml A1248

Inst ID: $g \mathrm{C} 2 . \mathbf{i}$
Quant Type: ESTD

Cal File: 004f0201.d

calibration Sample, Level: 3

Compound Sublist: AR1248.sub

Sample Matrix: WATER

\begin{tabular}{|c|c|c|c|c|c|c|c|}
\hline & & & & AMOUN & & & \\
\hline & & & & CAL-AMT & $\mathrm{ON}-\mathrm{COL}$ & & \\
\hline RT & EXP RT & DLT RT & RESPONSE & $n g$ ) & ng) & TARGET RANGE & RATIO \\
\hline$==$ & $=====$ & $== \pm==$ & $========$ & ======" & $\Rightarrow=====$ & 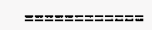 & $====$ \\
\hline $26 \mathrm{Ar}$ & roclor-12 & & & & CAS \# & $12672-29-6$ & \\
\hline 19.859 & 19.859 & 0.000 & 36113 & 0.10000 & 0 & & 100.00 \\
\hline 21.703 & 21.703 & 0.000 & 41653 & 0.10000 & 0 & $0.00-20.00$ & 115.34 \\
\hline 21.920 & 21.920 & 0.000 & 46227 & 0.10000 & 0 & $0.00-20.00$ & 128.01 \\
\hline & & verage o & Amounts = & & 0.095258 & & \\
\hline$\$ 29 T$ & etrachlo & $-m-x y l e$ & & & CAS \# & $877-09-8$ & \\
\hline 12.749 & 12.750 & -0.001 & 519640 & 0 & 0 & & 100.00 \\
\hline$\$ 30 \mathrm{D}$ & ecachlor & Jiphenyl & & & CAS \# & $2051-24-3$ & \\
\hline 43.062 & 43.059 & 0.003 & 215562 & 0 & 0 & & 100.00 \\
\hline
\end{tabular}


Data File: /chem/gc2.i/012398.b/005f0201.d

Data file: /chem/gc2.i/012398.b/005f0201.d

Lab Smp Id: 50ng/mi. A1248

Inj Date : 23-JAN-98 17:38

operator : Gerald Ross

Smp Info : 50ng/mI A1248

Misc Info: $50 \mathrm{ng} / \mathrm{ml} \mathrm{A} 1248$

Comment :

Method : /chem/gc2.i/012398.b/rcrapcb.m

Meth Date : 26-Jan-1998 15:30 gar

Cal Date : 23-JAN-98 17:38

Als bottle: 1

Dil Factor: 1.000

Integrator: HP Genie

Target Version: 3.10
Client Smp ID: 50ng/ml A1248

Inst ID: $g c 2 . i$
Quant Type: ESTD

Cal File: 005f0201.d

Calibration Sample, Level: 2

Compound sublist: AR1248. sub Sample Matrix: WATER

\begin{tabular}{|c|c|c|c|c|c|c|c|}
\hline & & & & AMOUN & NTS & & \\
\hline & & & & CAL-AMT & $\mathrm{ON}-\mathrm{COL}$ & & . \\
\hline RT & EXP RT & DLT RT & RESPONSE & ( $n g$ ) & ( $\quad n g)$ & TARGET RANGE & RATIO \\
\hline$=$ & $======$ & $== \pm==x=$ & $== \pm====$ & $==\pi===$ & $=======$ & 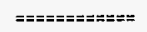 & $==0=$ \\
\hline $26 \mathrm{Ar}$ & roclor-1 & & & & CAS \#: & $12672-29-6$ & \\
\hline 19.859 & 19.859 & 0.000 & 19951 & 0 & 0 & & 100.00 \\
\hline 21.702 & 21.702 & 0.000 & 24687 & 0 & 0 & $0.00-20.00$ & 123.74 \\
\hline 21.920 & 21.920 & 0.000 & 27753 & 0 & 0 & $0.00-20.00$ & 139.11 \\
\hline & & verage of & Amounts = & & 0.055397 & & \\
\hline$\$ 29 \mathrm{~T}$ & etrachlo & $0-m-x y l e n$ & . & & CAS \#: & $877-09-8$ & \\
\hline 12.750 & 12.750 & 0.000 & 251549 & 0 & 0 & & 100.00 \\
\hline$\$ 30 \mathrm{DC}$ & ecachlor & biphenyl & & & CAS \#: & $2051-24-3$ & \\
\hline 43.064 & 43.059 & 0.005 & 152138 & 0 & 0 & & 100.00 \\
\hline
\end{tabular}


Column phase: Xti-5

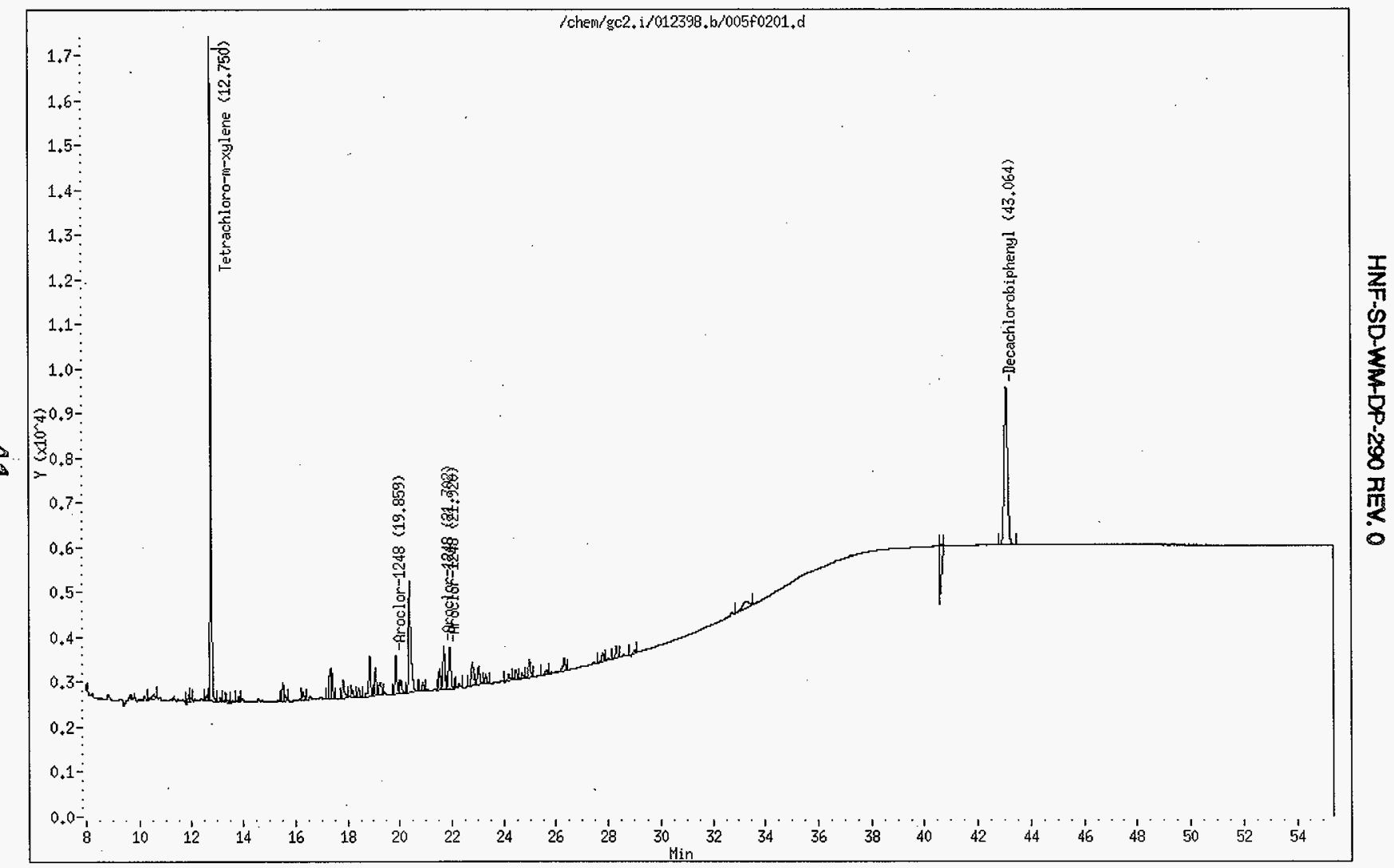




\section{HNF-SD-WA-DP-290 REV. 0}

Data File: /chem/gc2.i/012398.b/006f0201.d

Page 1

Report Date: 26-Jan-1998 15:30

\section{2-S Laboratory}

Data file : /chem/gc2.i/012398.b/006f0201.d

Lab Smp Id: 20 ng/ml A1248

Inj Date : 23-JAN-98 18:40

Operator : Gerald Ross

Client Smp ID: $20 \mathrm{ng} / \mathrm{ml}$ A1248

Smp Info : $20 \mathrm{ng} / \mathrm{ml}$ A1248

Inst ID: $g c 2 . i$

Misc Info : $20 \mathrm{ng} / \mathrm{ml}$ A 1248

comment :

Method : /chem/gc2.i/012398.b/rcrapcb:m

Meth Date : 26-Jan-1998 15:30 gar

Cal Date : 23-JAN-98 18:40

Als bottle: 1

Dil Factor: 1.000

Integrator: HP Genie

Target Version: 3.10

Quant Type: ESTD

Cal File: $006 f 0201 . d$

Calibration Sample, Level: 1

Compound Sublist: AR1248.sub

Sample Matrix: WATER

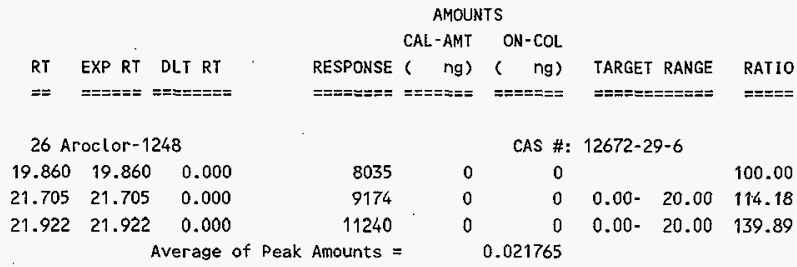

$\$ 29$ Tetrachloro-m-xylene

CAS \#: $877-09-8$

$\begin{array}{lllllll}12.751 & 12.750 & 0.001 & 146263 & 0 & 0 & 100.00\end{array}$

6 30 Decachlorobiphenyl

$\$ 30$ Decach Lorobiphenyl

CAS \#: $205.1-24-3$

$43.065 \quad 43.059 \quad 0.006$

98125

0

100.00 
HNF-SD-WM-DP-290 REY. 0

co

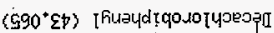
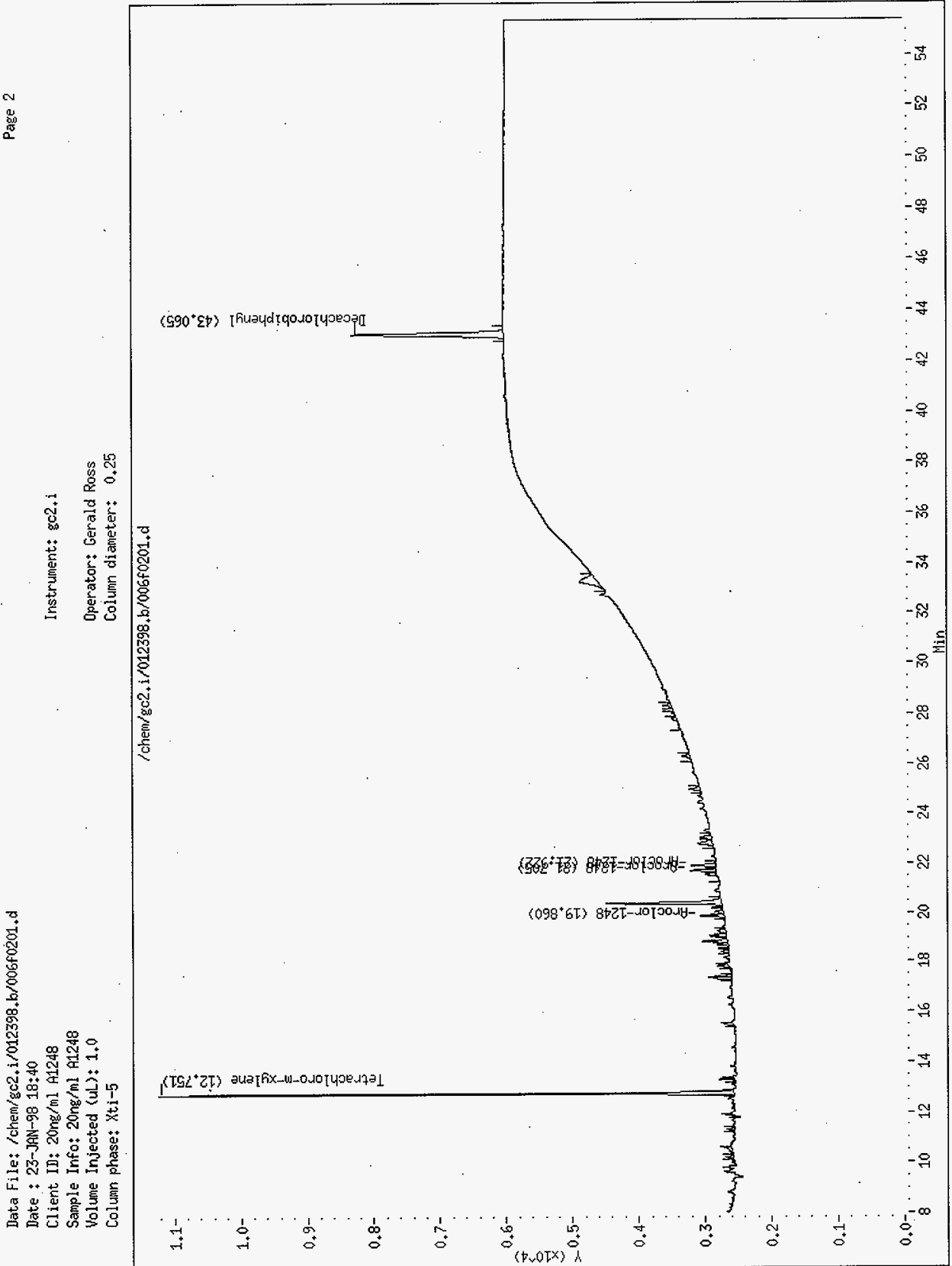
Data File: /chem/gc2.i/012398.b/007f0201.d

Report Date: 26-Jan-1998 15:31

\section{2-S Laboratory}

Data file : /chem/gc2.i/012398.b/007f0201.d

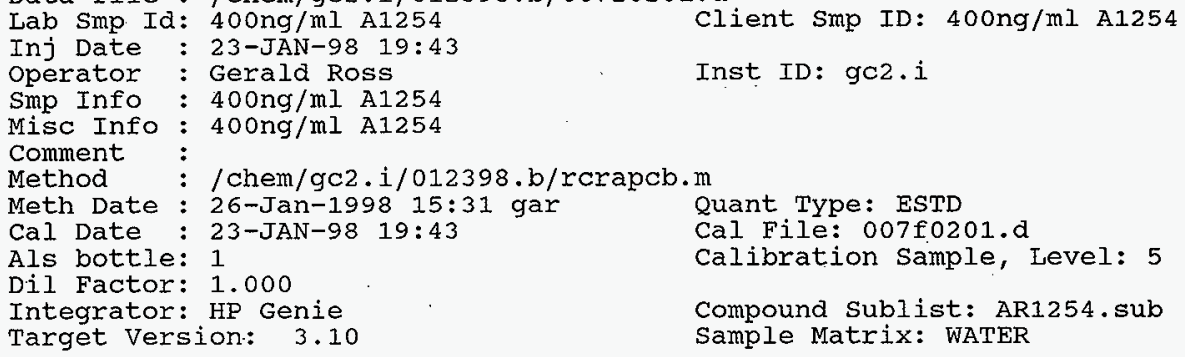

\begin{tabular}{|c|c|c|c|c|c|c|c|}
\hline & & & & AMOU: & & & \\
\hline & & & & CAL-AMT & $\mathrm{ON}-\mathrm{COL}$ & & \\
\hline RT & EXP RT & DLT RT & RESPONSE & ( $n g$ ) & ng) & TARGET RANGE & RATIO \\
\hline$==$ & $=====$ & $=======$ & 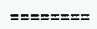 & $======$ & $======$ & $======-=====$ & $==$ \\
\hline $27 \mathrm{~A}$ & roclor-1 & 1254 & & & CAS \#: & $11097-69-1$ & \\
\hline 21.942 & 21.942 & $2 \quad 0.000$ & 197698 & 0.40000 & 0.45061 & & 100.00 \\
\hline 23.024 & 23.024 & 0.000 & 257336 & 0.40000 & 0.44308 & $0.00-20.00$ & 130.17 \\
\hline 24.972 & 24.972 & 0.000 & 240739 & 0.40000 & 0.45806 & $0.00-20.00$ & 121.77 \\
\hline & & Average of & Amounts $=$ & & 0.45059 & & \\
\hline $29 \mathrm{~T}$ & etrachlo & ro-m-xyler & & & CAS \#: & $877-09-8$ & \\
\hline 12.747 & 12.750 & -0.003 & 1947733 & 0.20000 & 0.20166 & & 100.00 \\
\hline$\$ 30 \mathrm{D}$ & ecachlor & obiphenyl & & & CAS \#: & $2051-24-3$ & \\
\hline 43.059 & 43.059 & 0.000 & 709291 & 0.20000 & 0.14315 & & 100.00 \\
\hline
\end{tabular}




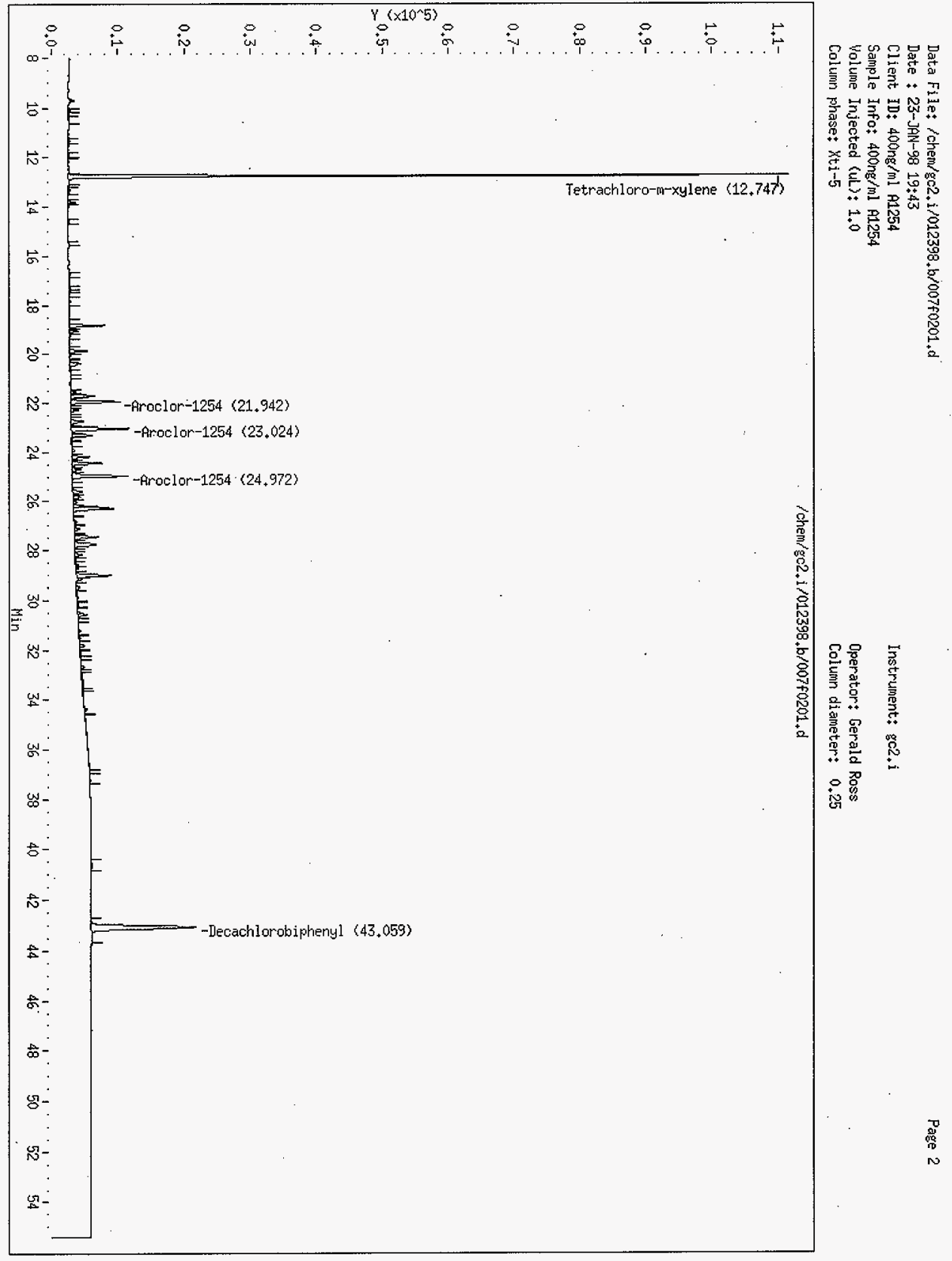


Data File: /chem/gc2.i/012398.b/008f0201.d

Data file:/chem/gc2.i/012398.b/008f0201.d

Lab Smp Id: $200 \mathrm{ng} / \mathrm{ml}$ A1254

Inj Date : 23-JAN-98 20:45

operator : Gerald Ross

Smp Info : $200 \mathrm{ng} / \mathrm{ml} \mathrm{A1254}$

Misc Info : 200ng/ml A1254

comment :

Method : /chem/gc2.i/012398.b/xcrapcb.m

Meth Date : 26-Jan-1998 15:31 gar

Cal Date : 23-JAN-98 20:45

Als bottle: 1

Dil Factor: 1.000

Integrator: HP Genie

Target Version: 3.10
Client Smp ID: 200ng/ml A1254

Inst ID: $g \circ 2 . i$

Quant Type: ESTD

Cal File: 008f0201.d

Calibration Sample, Level: 4

Compound Sublist: AR1254, sub Sample Matrix: WATER

\begin{tabular}{|c|c|c|c|c|c|c|c|}
\hline & & & & AMOUN & & & \\
\hline & & & & CAL-AMT & $\mathrm{ON}-\mathrm{COL}$ & & \\
\hline $\begin{array}{l}\text { RT } \\
==\end{array}$ & $\begin{array}{l}\text { EXP RT } \\
=====\end{array}$ & $\begin{array}{l}\text { LT RT } \\
=====\end{array}$ & $\begin{array}{l}\text { RESPONSE } \\
=======\end{array}$ & $\begin{array}{l}\text { ( } n g) \\
=======\end{array}$ & 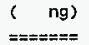 & $\begin{array}{l}\text { TARGET RANGE } \\
=========\end{array}$ & $\begin{array}{l}\text { RATIO } \\
====\end{array}$ \\
\hline $27 \mathrm{Ar}$ & roclor-12 & & & & CAS \#: & $11097-69-1$ & \\
\hline 21.946 & 21.946 & 0.000 & 77694 & 0.20000 & 0.17709 & & 100.00 \\
\hline 23.028 & 23.028 & 0.000 & 103140 & 0.20000 & 0.17759 & $0.00-20.00$ & 132.75 \\
\hline 24.976 & 24.976 & 0.000 & 90533 & 0.20000 & 0.17226 & $0.00-20.00$ & 116.53 \\
\hline & & erage o & Amounts $=$ & & 0.17564 & & \\
\hline
\end{tabular}

$\$ 29$ Tetrachloro-m-xylene

CAS \#: $877-09-8$

$12.749 \quad 12.750 \quad-0.001$

8769890.10000

100.00

$\$ 30$ Decachlorobiphenyl

CAS \#: 2051-24-3

$\begin{array}{lll}43.064 & 43.059 & 0.005\end{array}$

2585520.10000

0

100.00 


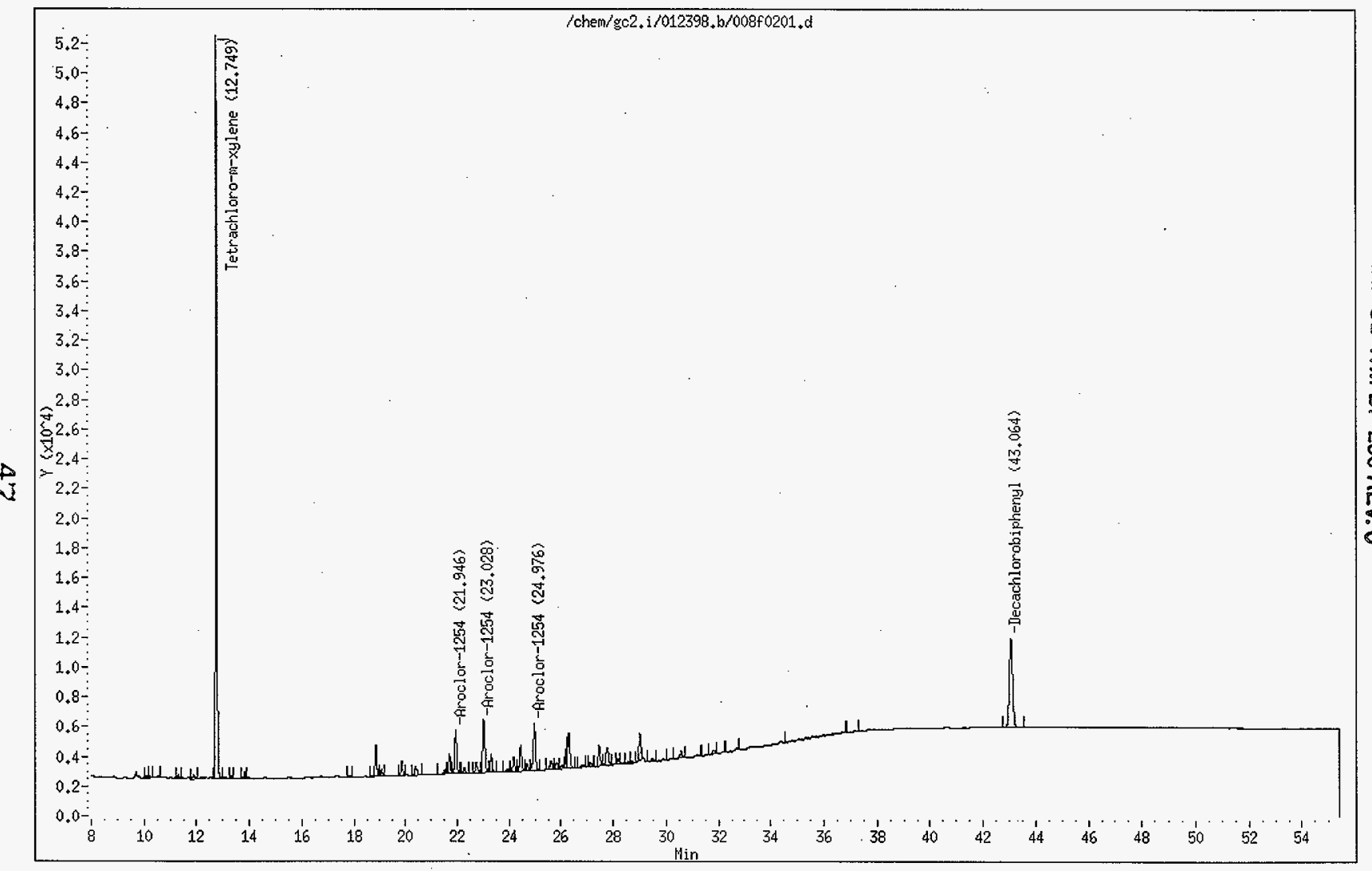


Data File: /chem/gc2.i/012398.b/009f0201.d

Page 1

Report Date: 26-Jan-1998 15:31

222-S Laboratory

Data file : /chem/gc2.i/012398.b/009f0201.d

Lab Smp Id: $100 \mathrm{ng} / \mathrm{ml}$ A 1254

Inj Date : 23-JAN-98 21:48

Operator : Gerald Ross

Smp Info : $100 \mathrm{ng} / \mathrm{ml}$ A 1254

Misc Info: $100 \mathrm{ng} / \mathrm{ml}$ A 1254

comment :

Method : /chem/gc2.i/012398.b/rcrapcb.m

Meth Date : 26-Jan-1998 15:31 gar Quant Type: ESTD

Cal Date : 23-JAN-98 21:48

Cal. File: 009f0201.d

Als bottle: 1

Calibration Sample, Level: 3 .

Dil Factor: 1.000

Integrator: HP Genie

Target Version: 3.10

Compound Sublist: AR1254.sub

Sample Matrix: WATER

\begin{tabular}{|c|c|c|c|c|c|c|c|}
\hline & & & & AMOUN & & & \\
\hline & & & & CAL-AMT & $\mathrm{ON}-\mathrm{COL}$ & & \\
\hline RT & EXP RT & $L T R T$ & RESPONSE ( & ( $\mathrm{ng}$ ) & ( $n g$ ) & TARGET RANGE & RATIO \\
\hline$==$ & $\Rightarrow=== \pm=$ & $==== \pm==$ & $=$ & $===-= \pm=$ & $==E====$ & $======8=== \pm=$ & $== \pm==$ \\
\hline $27 \mathrm{Ar}$ & oclor -12 & & & & CAS \#: & $11097-69-1$ & \\
\hline 21.947 & 21.947 & 0.000 & 183190 & 0.10000 & 0 & & 100.00 (a) \\
\hline 23.031 & 23.031 & 0.000 & 230210 & 0.10000 & 0 & $0.00-\quad 20.00$ & 125.67 \\
\hline 24.978 & 24.978 & 0.000 & 204990 & 0.10000 & 0 & $0.00-20.00$ & 111.90 \\
\hline . & & verage of Peak & Amounts $=$ & & 0.040132 & & \\
\hline$\$ 29 \mathrm{Te}$ & trachlor & $0-m-x y l e n e$ & & & CAS \#: & $877-09-8$ & \\
\hline 12.750 & 12.750 & 0.000 & 379483 & 0 & 0 & & 100.00 \\
\hline$\$ 30 \mathrm{De}$ & cachloro & biphenyl & & & CAS \#: & $2051-24-3$ & \\
\hline 43.066 & 43.059 & 0.007 & 183838 & 0 & 0 & & 100.00 \\
\hline
\end{tabular}

\section{QC Flag Legend}

a - Target compound detected but, quantitated amount Below Limit of quantitation(BLOQ). 


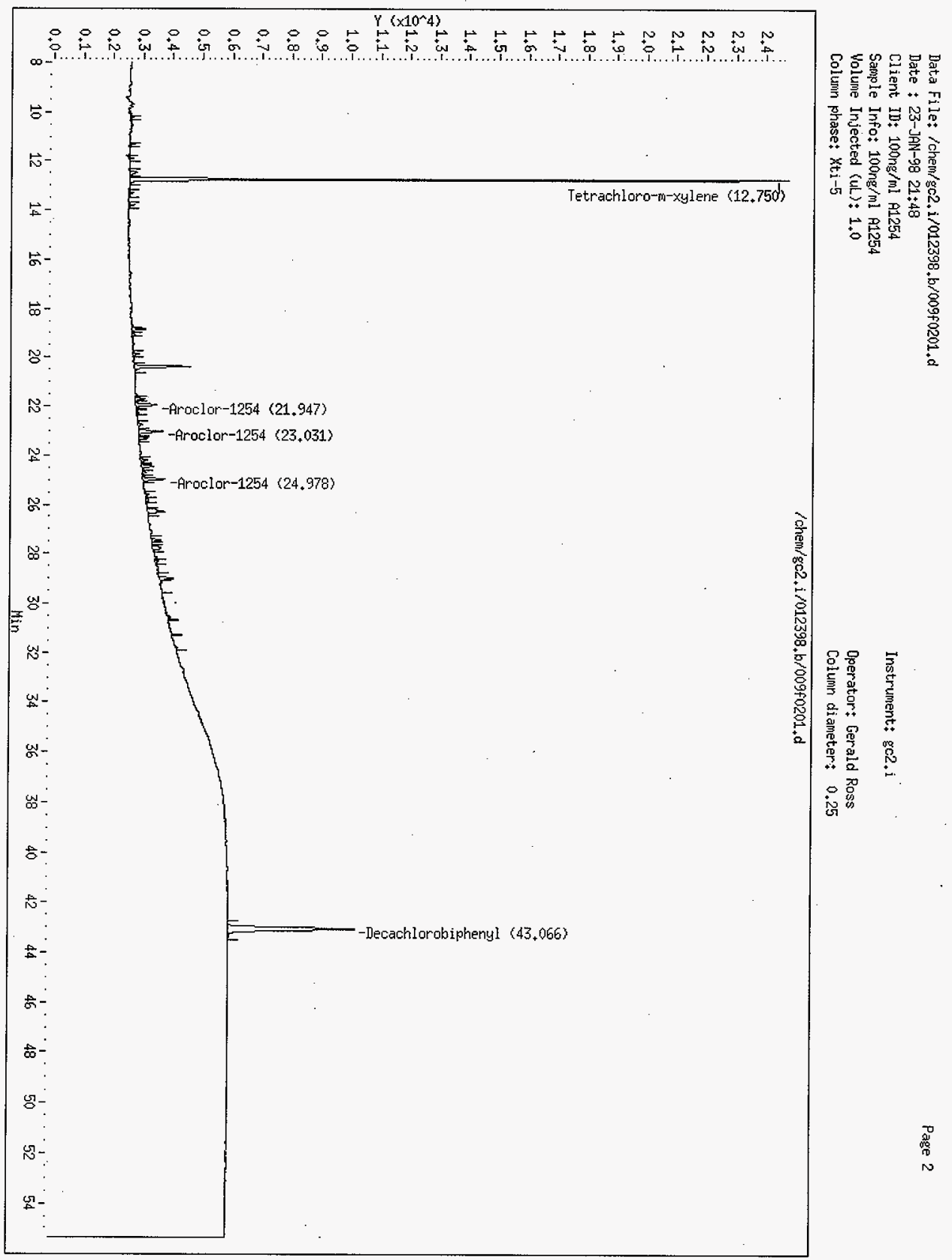




\section{HNF-SD-WRADP-290 REV. 0}

Data File: /chem/gc2.i/012398.b/010f0201.d

Report Date: 26-Jan-1998 15:31

Page 1

\section{2-S Laboratory}

Data file : /chem/gc2.i/012398.b/010f0201.d

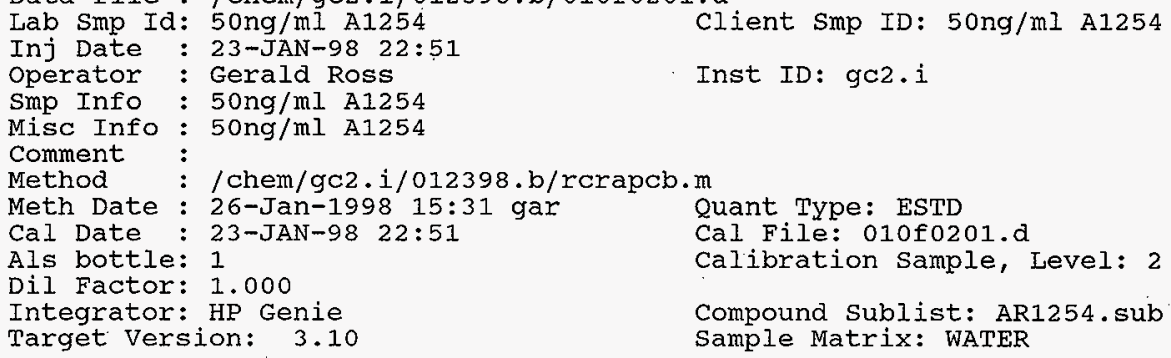

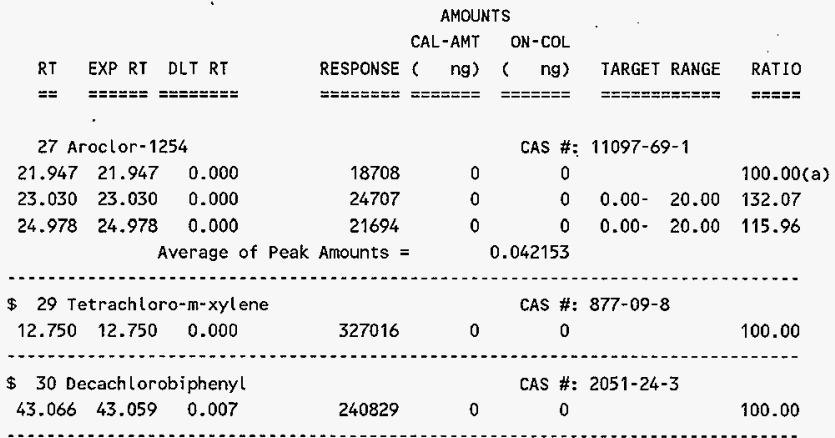

QC Flag Legend

a - Target compound detected but, quantitated amount Below Limit of Quantitation(BLOQ). 


\section{HNF-SD-WRADP-290 REV. 0}

Data File: /chem/gc2.i/012398.b/011f0201.d

Report Date: 26-Jan-1998 15:31

\section{2-S Laboratory}

Data file : /chem/gc2.i/012398.b/011f0201.d

Lab Smp Id: $20 \mathrm{ng} / \mathrm{ml}$ A1254

Inj Date : 23-JAN-98 23:53

Operator : Gerald Ross

Smp Info : $20 \mathrm{ng} / \mathrm{ml} \mathrm{A1254}$

Misc Info: $20 \mathrm{ng} / \mathrm{ml} \mathrm{A} 1254$

Comment

Method

Meth Date : 26-Jan-1998 15:31 gar

Cal Date : 23-JAN-98 23:53

Als bottle: 1

Dil Factor: 1.000

Integrator: HP Genie

Target Version: 3.10
Client Smp ID: $20 \mathrm{ng} / \mathrm{ml}$ A1254

Inst ID: gC2.i

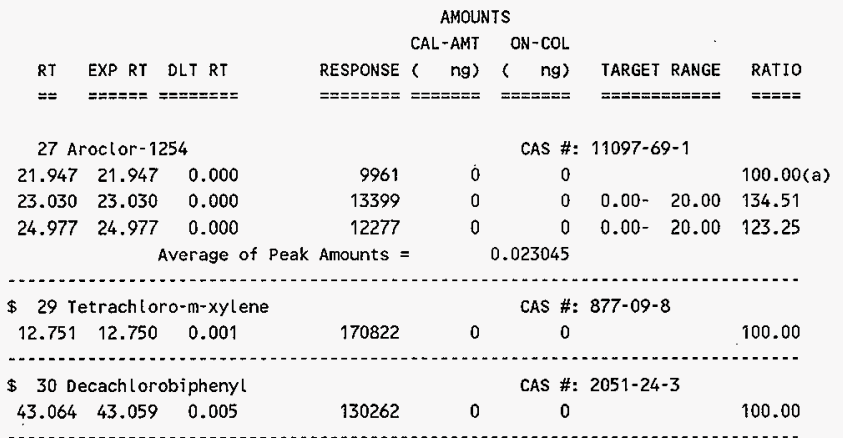

QC Flag Legend

a - Target compound detected but, quantitated amount Below Limit of quantitation(BLOQ).

Quant Type: ESTD

Cal File: 011f0201.d

Calibration Sample, Level: 1

Compound Sublist: AR1254.sub Sample Matrix: WATER 


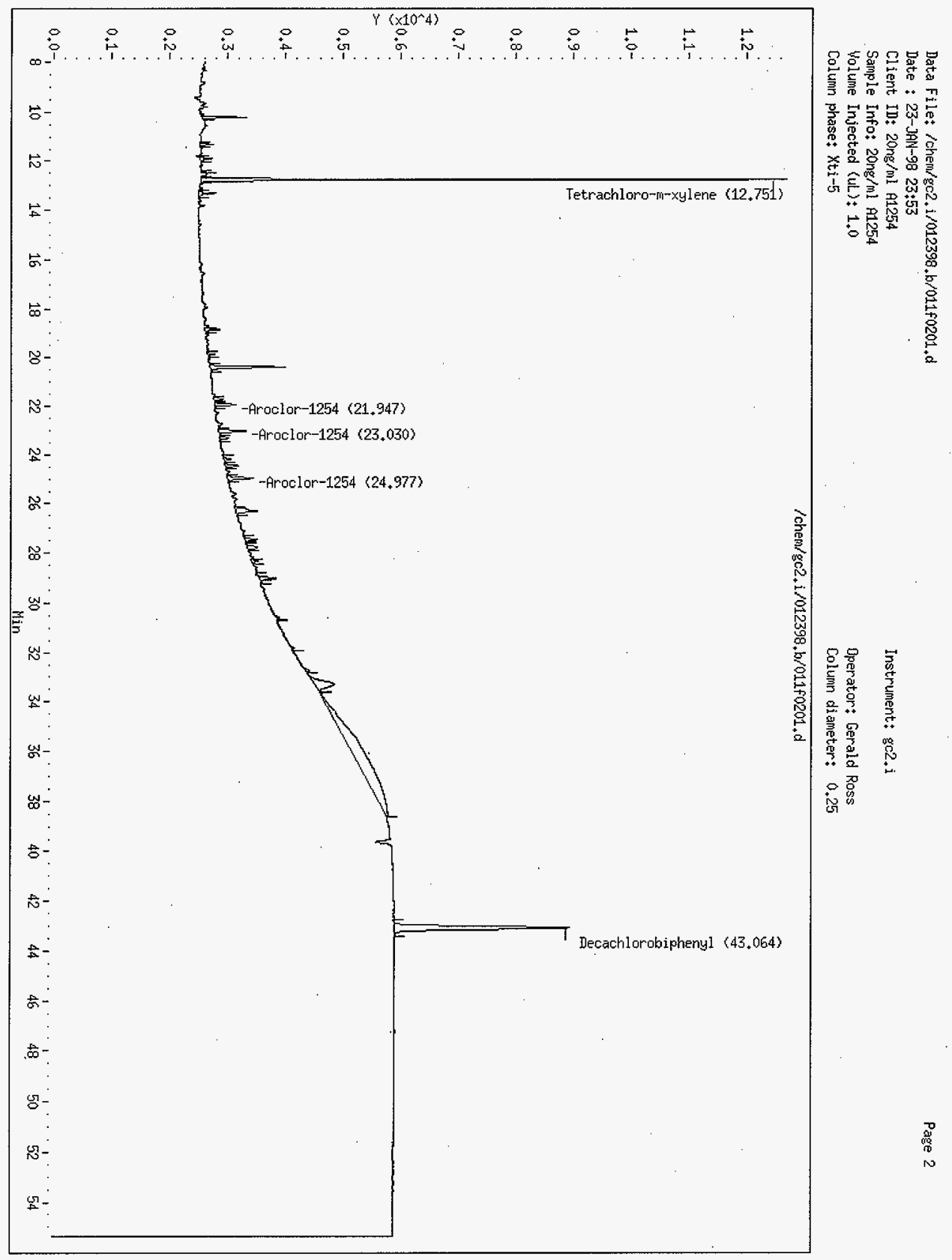


Data File: /chem/gc2.i/012398.b/012f0201.d

Page 1

Report Date: 27-Jan-1998 07:12

\section{2-S Iaboratory}

Data file : /chem/gc2.i/012398.b/012f0201.d

Lab Smp Id: $400 \mathrm{ng} / \mathrm{ml}$ A 1660

Client Smp ID: $400 \mathrm{ng} / \mathrm{ml}$ A1660

Inj Date : 24-JAN-1998 00:56

Operator : Gerald Ross

Inst ID: $g \mathrm{C} 2 . i$

Smp Info : 400 $\mathrm{ng} / \mathrm{ml}$ A1660

Misc Info: $400 \mathrm{ng} / \mathrm{ml}$ A1660

comment

Method : /chem/gc2.i/012398.b/rcrapcb.m

Meth Date : 27-Jan-1998 07:12 gar

Cal Date : 24-JAN-1998 00:56

Als bottle: 1

Dil Factor: 1.000

Integrator: HP Genie

Target Version: 3.10

Quant Type: ESTD

Cal File: 012f0201.d

Calibration Sample, Level: 5

Compound Sublist: AR1660.sub Sample Matrix: WATER

\begin{tabular}{|c|c|c|c|c|c|c|c|}
\hline \multirow[b]{3}{*}{ RT } & \multirow[b]{3}{*}{ EXP RT } & \multicolumn{6}{|c|}{ AMOUNTS } \\
\hline & & \multicolumn{3}{|r|}{ CAL-AMT } & $\mathrm{ON}-\mathrm{COL}$ & \multirow[b]{2}{*}{ TARGET RANGE } & \multirow{3}{*}{ RATI0 } \\
\hline & & DLT RT & RESPONSE & ( $\mathrm{ng})$ & ( $n g)$ & & \\
\hline$==$ & $=====$ & $======$ & $===ニ=\pi==$ & $======$ & $======$ & 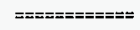 & \\
\hline \multicolumn{3}{|c|}{22 Aroclor -1016} & & \multicolumn{3}{|c|}{ CAS \#: $12674-11-2$} & \\
\hline 15.496 & 15.496 & 0.000 & 138455 & 0.40000 & 0.36895 & & 100.00 \\
\hline 17.305 & 17.305 & 0.000 & 162225 & 0.40000 & 0.36165 & $0.00-20.00$ & 117.17 \\
\hline 17.385 & 17.385 & 0.000 & 206544 & 0.40000 & 0.37433 & $0.00-20.00$ & 149.18 \\
\hline \multicolumn{3}{|c|}{ Average of Peak } & Amounts $=$ & \multicolumn{2}{|r|}{0.36831} & & \\
\hline \multicolumn{3}{|c|}{28 Aroclor -1260} & & \multicolumn{3}{|c|}{ CAS \#: $11096-82-5$} & \\
\hline 26.210 & 26.210 & 0.000 & 182297 & 0.40000 & 0.33479 & & 100.00 \\
\hline 27.445 & 27.445 & 0.000 & 179803 & 0.40000 & 0.32852 & $0.00-20.00$ & 98.63 \\
\hline 29.005 & 29.005 & 0.000 & 154337 & 0.40000 & 0.31699 & $0.00-20.00$ & 84.66 \\
\hline \multicolumn{3}{|c|}{ Average of Peak } & Amounts $=$ & \multicolumn{2}{|r|}{0.32677} & & \\
\hline \multicolumn{3}{|c|}{29 Tetrachloro-m-xylene } & & \multicolumn{4}{|c|}{ CAS \#: $877-09-8$} \\
\hline 12.746 & 12.746 & 0.000 & 1751171 & 0.20000 & 0.22720 & & 100.00 \\
\hline \multicolumn{4}{|c|}{30 Decachlorobiphenyl } & \multirow{2}{*}{\multicolumn{3}{|c|}{$\begin{array}{ll} & \text { CAS \#: } 2051-24-3 \\
0.20000 & 0.16532\end{array}$}} & \\
\hline 43.059 & 43.059 & 0.000 & 678257 & & & & 100.00 \\
\hline
\end{tabular}




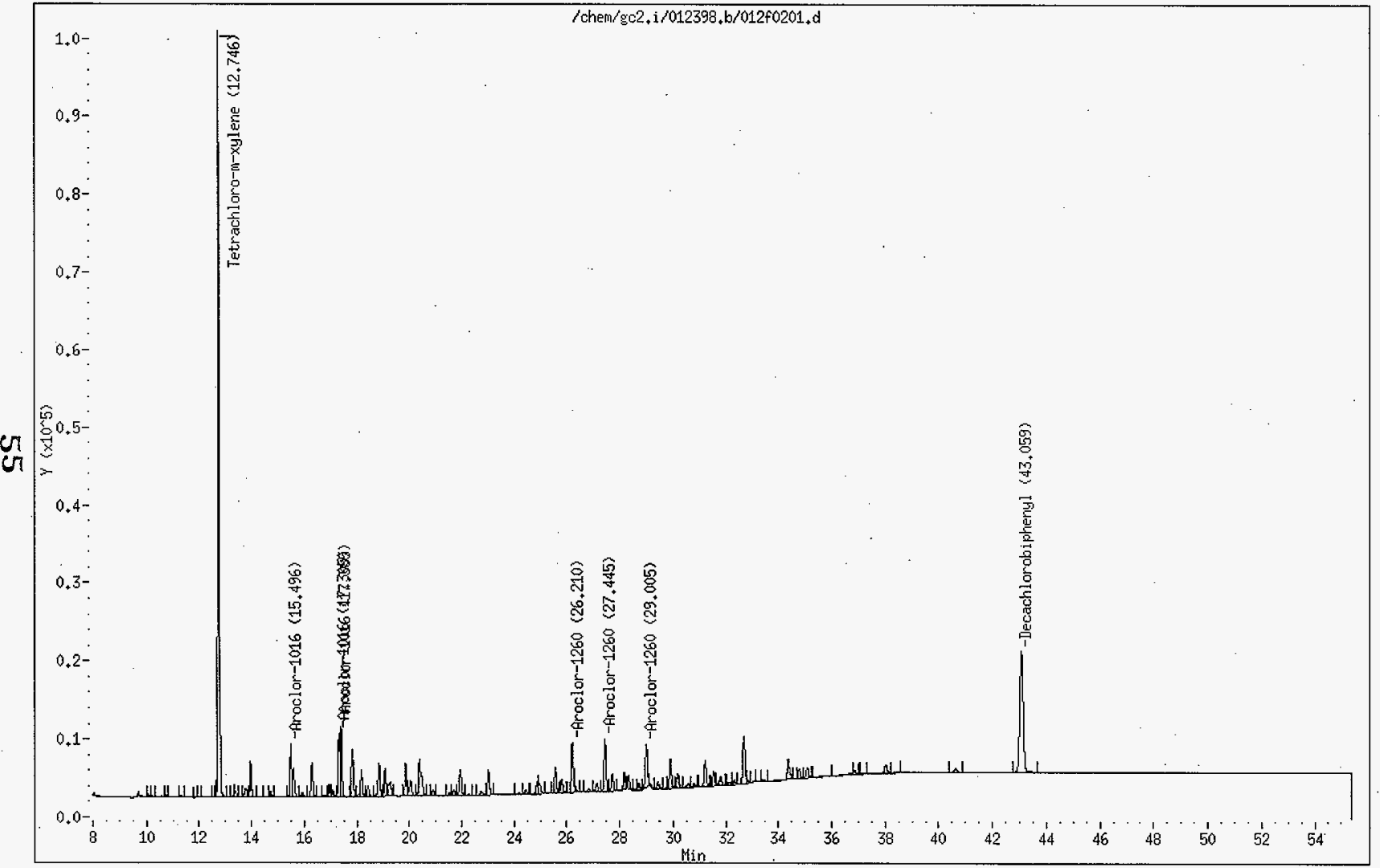


Data file:/chem/gc2.i/012398.b/013f0201.d

Lab Smp Id: $200 \mathrm{ng} / \mathrm{ml}$ Al660

Inj Date : 24-JAN-1998 01:58

operator : Gerald Ross

Smp Info : $200 \mathrm{ng} / \mathrm{ml}$ A1660

Misc Info: $200 \mathrm{ng} / \mathrm{ml}$ A1660

comment

Method : /chem/gc2.i/012398.b/rcrapcb.m

Meth Date : 27-Jan-1998 07:12 gar

Cal Date : 24-JAN-1998 01:58

Als bottle: 1

Dil. Factor: 1.000

Integrator: HP Genie

Target Version: 3.10
Client Smp ID: $200 \mathrm{ng} / \mathrm{ml}$ A1660

Inst ID: $9 \mathrm{C} 2 \cdot \mathrm{i}$
Quant Type: ESTD

Cal File: 013f0201.d

Calibration Sample, Level: 4

Compound Sublist: AR1660.sub Sample Matrix: WATER

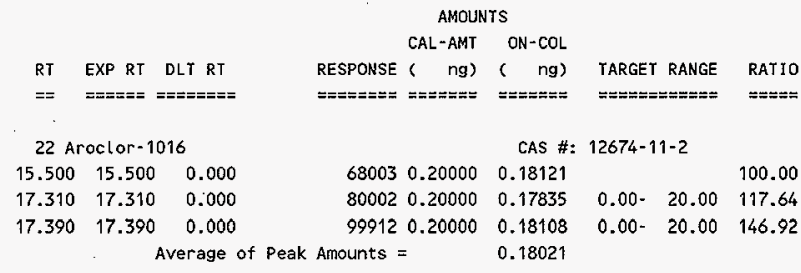

28 Aroclor -1260

$\begin{array}{lll}26.213 & 26.213 & 0.000 \\ 27.449 & 27.449 & 0.000 \\ 29.007 & 29.007 & 0.000\end{array}$ Average of Peak Amounts =
CAS \#: $11096-82-5$

$\begin{array}{rrrrrr}98848 & 0.20000 & 0.18154 & & & 100.00 \\ 100683 & 0.20000 & 0.18396 & 0.00- & 20.00 & 101.86 \\ 86540 & 0.20000 & 0.17774 & 0.00- & 20.00 & 87.55\end{array}$

0.18108

29 Tetrachloro-m-xylene

$\begin{array}{lll}12.748 & 12.748 & 0.000\end{array}$

CAS \#: $877-09-8$

30 Decach lorobiphenyl

$\begin{array}{lll}43.059 & 43.059 \quad 0.000\end{array}$

$878818 \quad 0.10000 \quad 0.11402$

100.00

CAS \#: 2051-24-3

$\begin{array}{lll}455364 & 0.10000 & 0.11099\end{array}$

100.00 


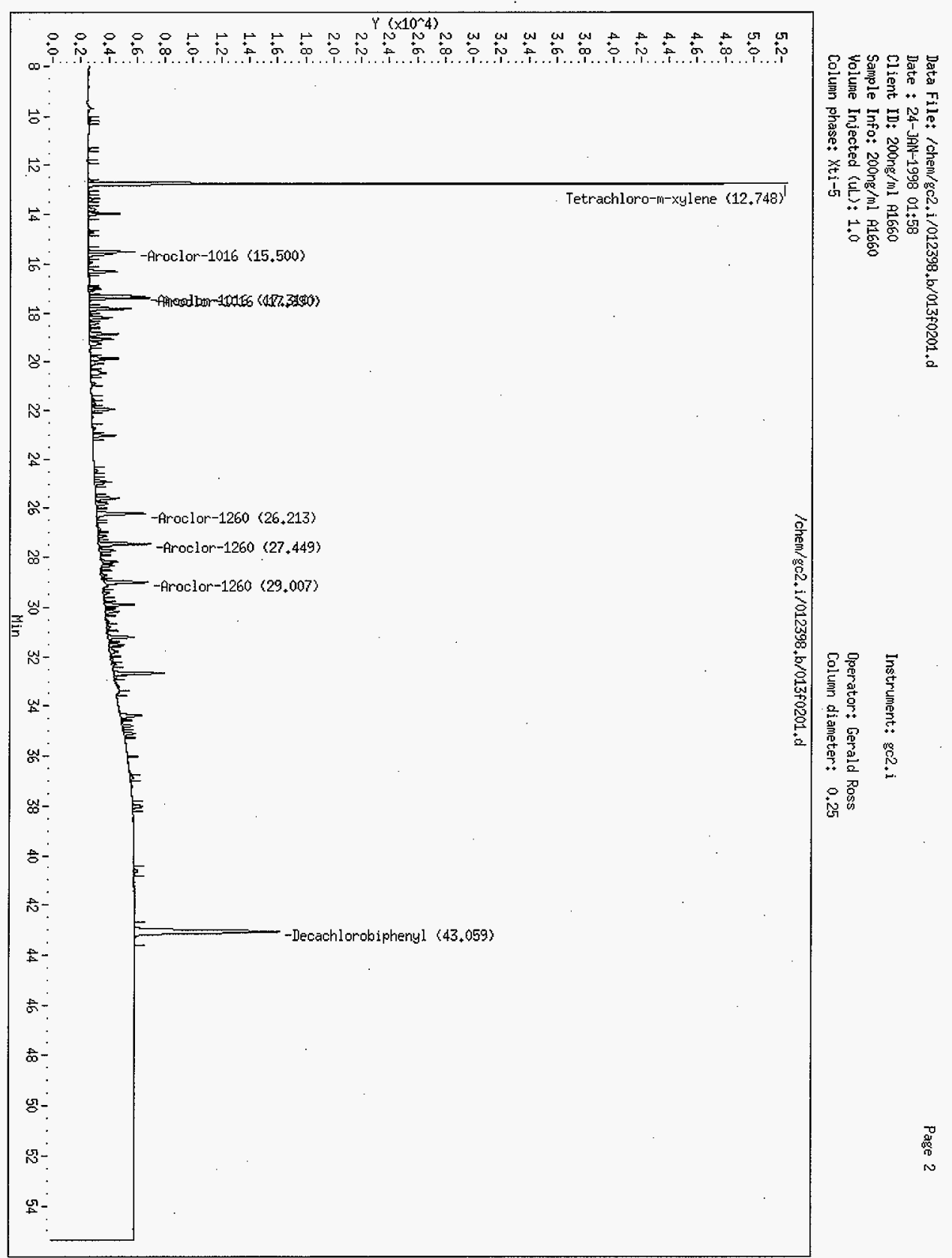




\section{HNF-SD-WAHDP-290 REV. 0}

Data File: /chem/gc2,i/012398.b/014f0201.d

Page 1

Report Date: 27-Jan-1998 07:12

\section{2-S Laboratory}

Data file: /chem/gc2.i/012398.b/014f0201.d

Lab Smp Id: $100 \mathrm{ng} / \mathrm{ml}$ A 1660

Client Smp ID: 100ng/ml A1660

Inj Date : 24-JAN-1998 03:01

Operator : Gerald Ross

Inst ID: $g \mathrm{c} 2 . \mathrm{i}$

Smp Info : $100 \mathrm{ng} / \mathrm{ml}$ A1660

Misc Info : $100 \mathrm{ng} / \mathrm{ml}$ A 1660

comment

Method : /chem/gc2.i/012398.b/rcrapcb.m

Meth Date : 27-Jan-1998 07:12 gar

Cal Date : 24-JAN-1998 03:01

Als bottle: 1

Dil Factor: 1.000

Integrator: HP Genie

Target Version: 3.10

Quant Type: ESTD

Cal File: 014f0201.d

Calibration Sample, Level: 3

Compound Sublist: AR1660.sub

Sample Matrix: WATER

\begin{tabular}{|c|c|c|c|c|c|c|c|}
\hline \multirow[b]{3}{*}{ RT } & \multirow[b]{3}{*}{ EXP RT } & \multicolumn{6}{|c|}{ AMOUNTS } \\
\hline & & \multirow[b]{2}{*}{ DLT RT } & & CAL-AMT & $\mathrm{ON}-\mathrm{COL}$ & \multirow[b]{2}{*}{ TARGET RANGE } & \multirow[b]{2}{*}{ RATIO } \\
\hline & & & RESPONSE & $\mathrm{ng})$ & $\mathrm{ng})$ & & \\
\hline$==$ & \multicolumn{2}{|c|}{ 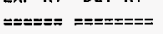 } & $==ニ=ニ===$ & $======$ & $======$ & 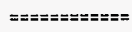 & $====0$ \\
\hline \multicolumn{3}{|c|}{22 Aroclor -1016} & & \multicolumn{3}{|c|}{ CAS \#: $12674-11-2$} & \\
\hline 15.500 & 15.500 & 0.000 & 41833 & 0.10000 & 0.11147 & & 100.00 \\
\hline 17.310 & 17.310 & 0.000 & 49043 & 0.10000 & 0.10933 & $0.00-20.00$ & 117.24 \\
\hline 17.390 & 17.390 & 0.000 & 59778 & 0.10000 & 0.10834 & $0.00-20.00$ & 142.90 \\
\hline \multicolumn{4}{|c|}{ Average of Peak Amounts = } & & 0.10972 & & \\
\hline
\end{tabular}

28 Aroclor -1260

$26.212 \quad 26.212 \quad 0.000$

$27.449 \quad 27.449 \quad 0.000$

$29.007 \quad 29.007 \quad 0.000$

Average of Peak Amounts =
CAS \#: $11096-82-5$

$\begin{array}{lll}56984 & 0.10000 & 0.10465\end{array}$

$\begin{array}{lll}56706 & 0.10000 & 0.10361\end{array}$

0.10758

0.10528
100.00

$0.00-20.00 \quad 99.51$

$0.00-20.00 \quad 91.91$

29 Tetrachloro-m-xylene

CAS \#: 877-09-8

$\begin{array}{lll}12.749 & 12.749 \quad 0.000\end{array}$

30 Decachlorobiphenyl

$\begin{array}{lll}43.059 & 43.059 \quad 0.000\end{array}$

545372

0

0

100.00

CAS \#: 2051-24-3

$\begin{array}{llll}223153 & 0 & 0 & 100.00\end{array}$




\section{$6 \mathrm{~s}$}

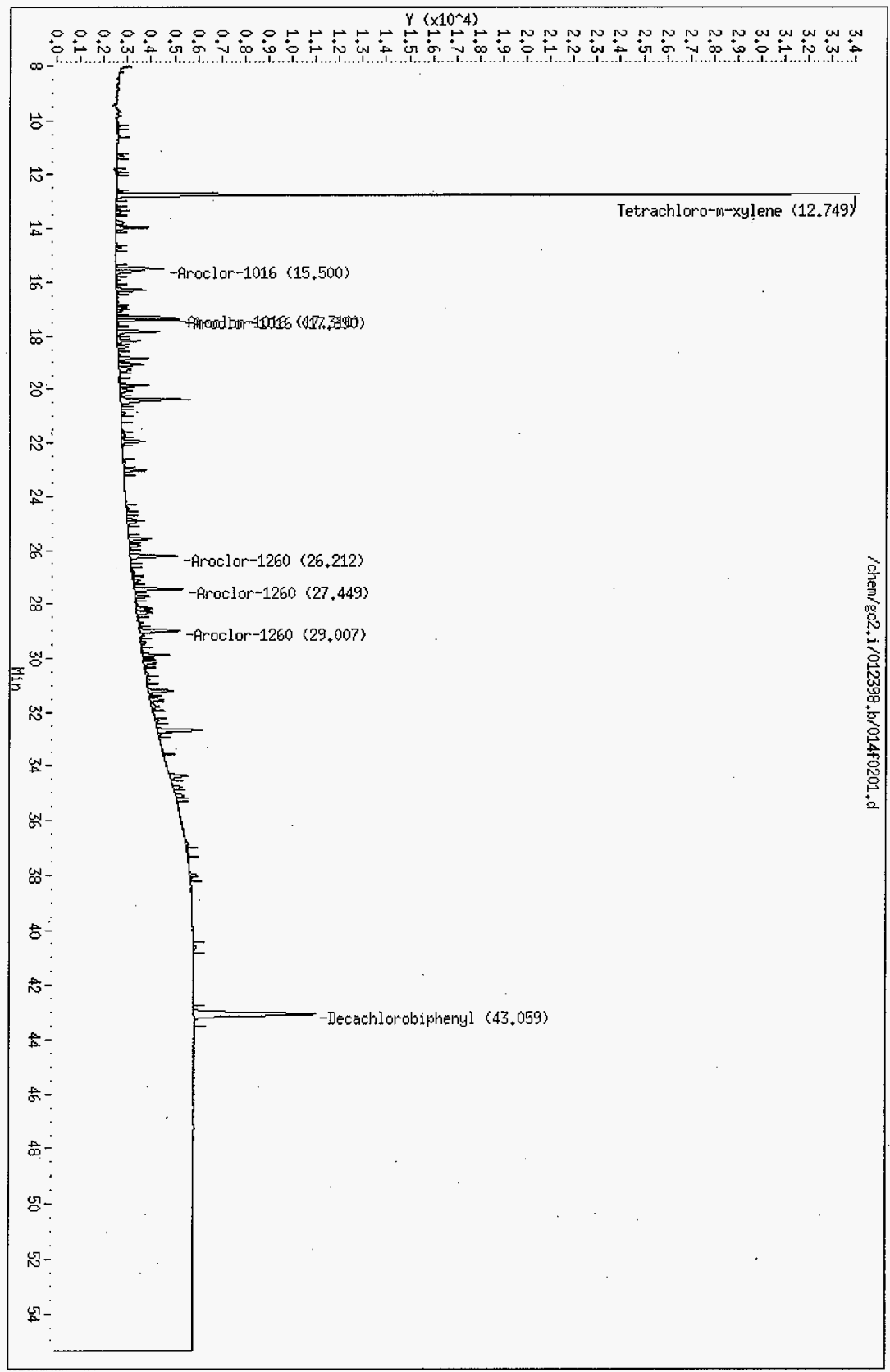

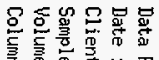

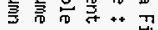
올골역

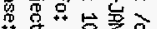

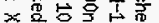
至的总总 ज舟要 喀 总量吊 8

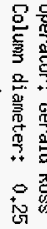

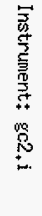


Data File: /chem/gc2,i/012398.b/015f0201.d

Report Date: 27-Jan-1998 07:12

Page 1

\section{2-S Laboratory}

Data file : /chem/gc2.i/012398.b/015f0201.d

Lab Smp Id: $50 \mathrm{ng} / \mathrm{ml}$ A1660

Inj Date : 24-JAN-1998 04:04

Operator : Gerald Ross

Simp Info : 50ng/ml A1660

Misc Info : $50 \mathrm{ng} / \mathrm{ml}$ A 1660

comment

Method

/chem/gc2.i/012398.b/rcrapcb.m

Meth Date : 27-Jan-1998 07:12 gar

Cal Date : 24-JAN-1998 04:04

Als bottle: 1

Dil Factor: 1.000

Integrator: HP Genie

Target Version: 3.10

Client Smp ID: 50ng/ml A1660

Inst ID: $g c 2 . i$

Quant Type: ESTD

Cal File: 015f0201.d

Calibration Sample, Level: 2

Compound Sublist: AR1660.sub Sample Matrix: WATER

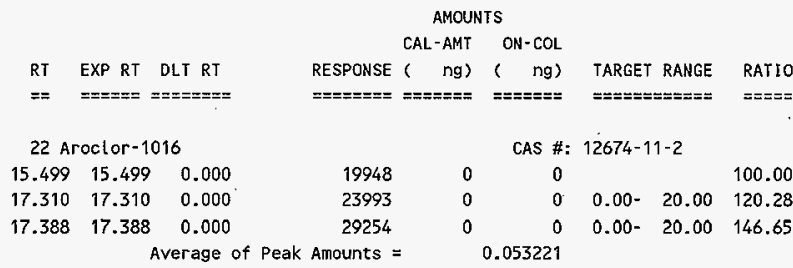

28 Aroclor -1260

\begin{tabular}{cccc}
26.212 & 26.212 & 0.000 & 29331 \\
27.449 & 27.449 & 0.000 & 29755 \\
29.006 & 29.006 & 0.000 & 25124 \\
& \multicolumn{4}{c}{ Average of Peak Amounts = }
\end{tabular}

29 Tetrachloro-m-xylene

$\begin{array}{lll}12.748 & 12.748 & 0.000\end{array}$

30 Decachlorobiphenyl

$43.060 \quad 43.060 \quad 0.000$

43.060 43.060 0.000
CAS \#: $11096-82-5$

$\begin{array}{rrrrr}0 & 0 & & & 100.00 \\ 0 & 0 & 0.00- & 20.00 & 101.45 \\ 0 & 0 & 0.00- & 20.00 & 85.66 \\ 0.053278 & & & \end{array}$

CAS \#: $877-09-8$

100.00

CAS \#: 2051-24-3

$0 \quad 0.100 .00$




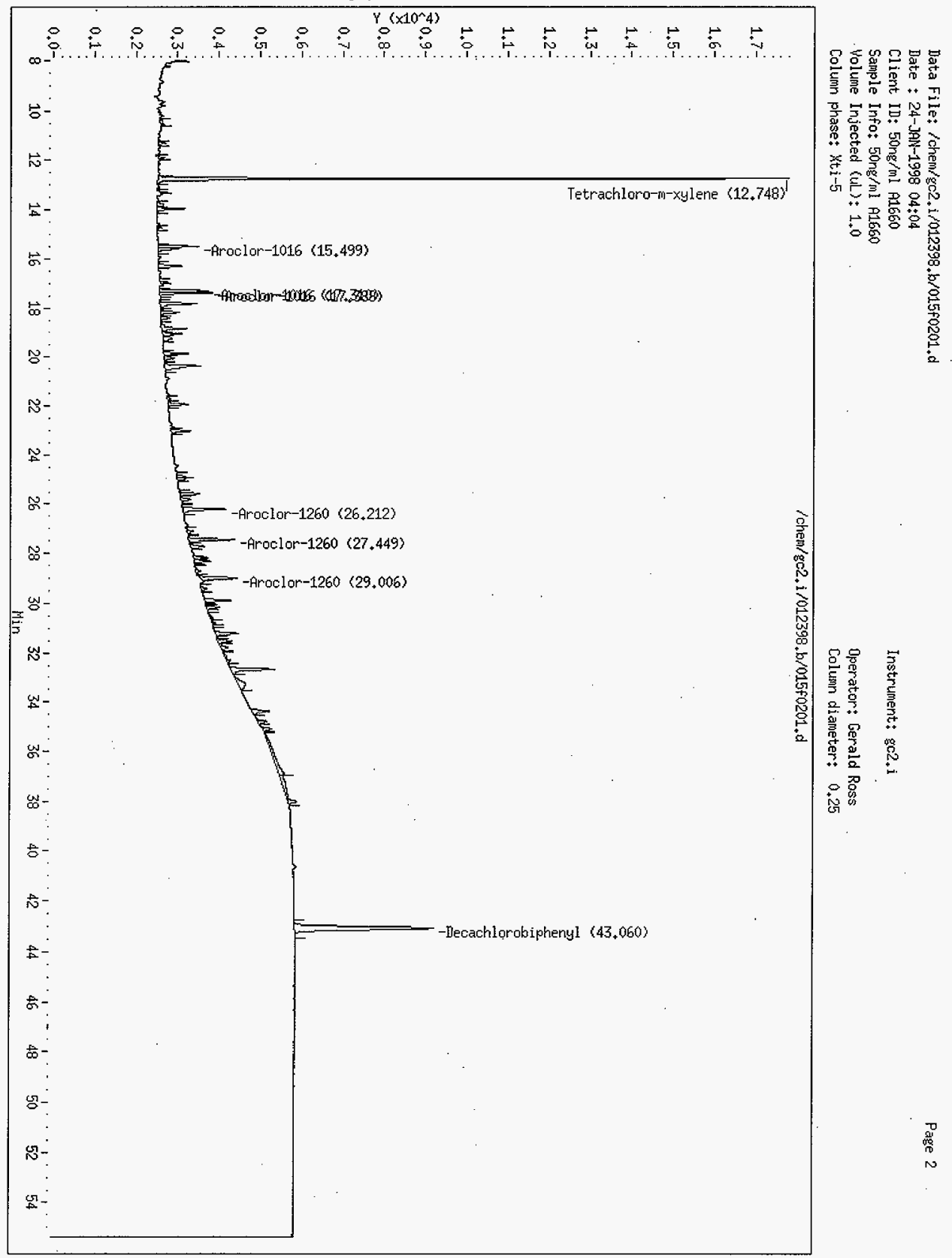




\section{HNF-SD-WMADP-290 REV. 0}

Data File: /chem/gc2.i/012398.b/016f0201.d

Report Date: 27-Jan-1998 07:13

\section{2-S Laboratory}

Data file:/chem/gc2.i/012398.b/016f0201.d

Lab smp Id: $20 \mathrm{ng} / \mathrm{mI}$ A 1660

Inj Date : 24-JAN-1998 05:06

Operator : Gerald Ross

Smp Info : $20 \mathrm{ng} / \mathrm{ml}$ A 1660

Misc Info : $20 \mathrm{ng} / \mathrm{ml}$ A1660

Comment

Method

/chem/gc2.i/012398.b/rcrapcb.m

Meth Date : 27-Jan-1998 07:13 gar

Cal Date : 24-JAN-1998 05:06

Als bottle: 1

Dil Factor: 1.000

Integrator: HP Genie

Target Version: 3.10
Client Smp ID: 2 ong/ml A1660

Inst ID: $9 \mathrm{c} 2, \mathrm{i}$

Quant Type: ESTD

Cal File: 016f0201.d

Calibration Sample, Level: 1

Compound sublist: AR1660.sub Sample Matrix: WATER

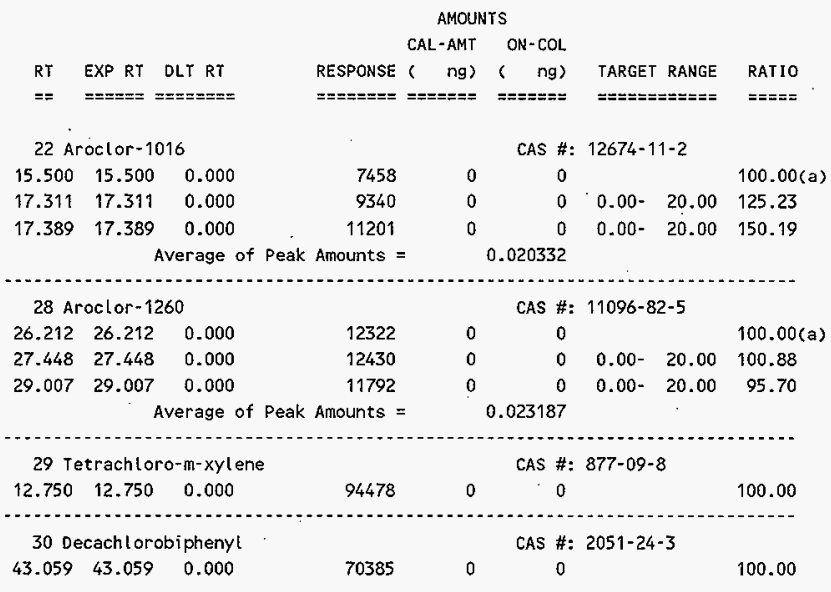

QC Flag Legend

a - Target compound detected but, quantitated amount Below Limit of quantitation(BLOQ). 
Data File: /chem/gc2,i/012398.b/016fo201,d

Date : 24-JAN-1998 05;06

Client II: 20/ng/ml A1660

Sample Info: 20ng/ml A1660

Volume Injected $(u L): 1.0$

Instrument: gc2.i

Column phase: Xti-5

Operator: Cerald Ross

Column dianeter: 0.25

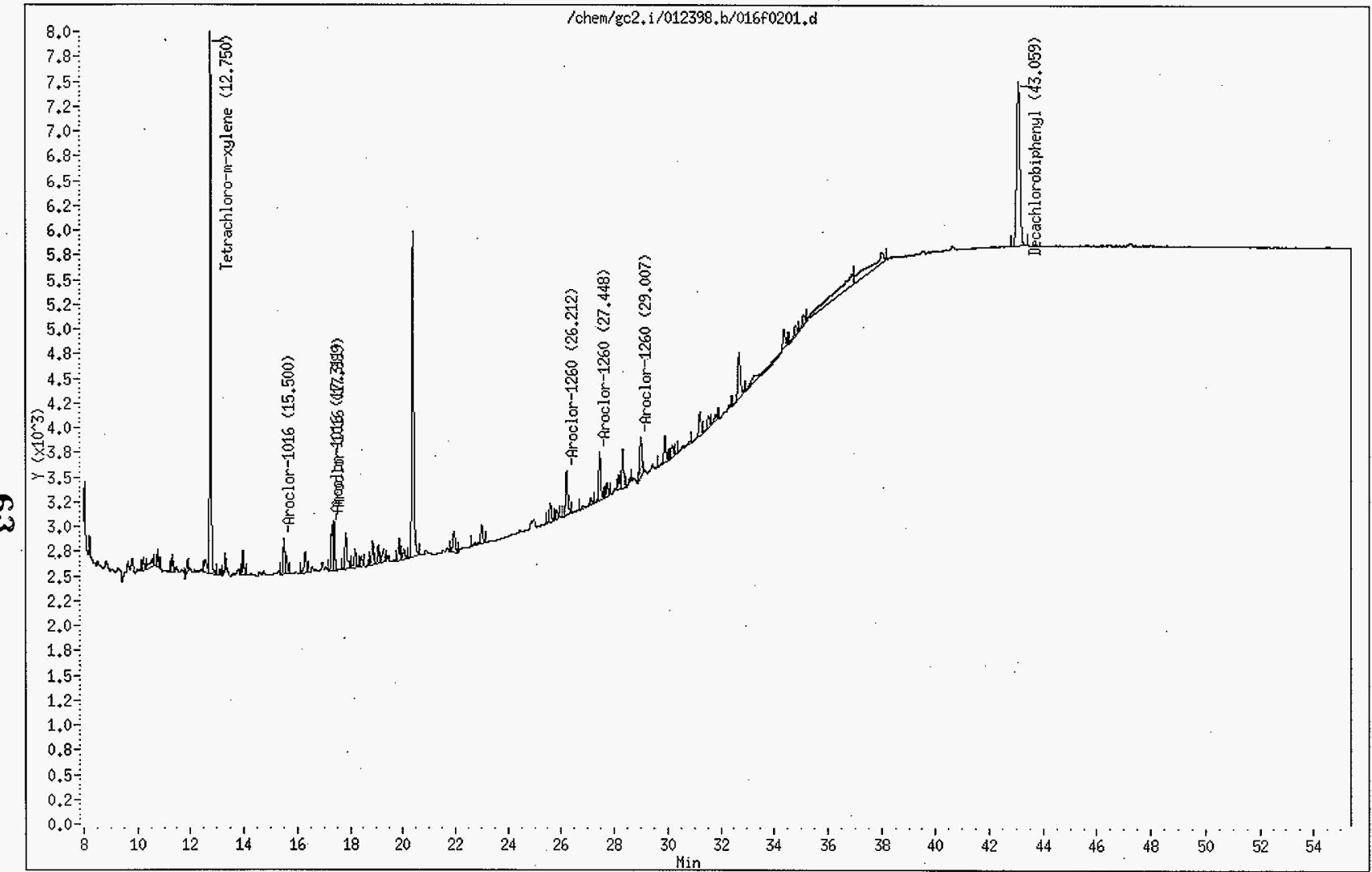

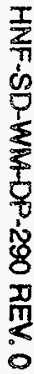


Data file : /chem/gc2.i/012398.b/017f0201.d

Lab Smp Id: $200 \mathrm{ng} / \mathrm{ml}$ A 1221

Inj Date : 24-JAN-1998 06:09

Operator : Gerald Ross

Smp Info : $200 \mathrm{ng} / \mathrm{ml}$ A1221

Misc Info: 200ng/ml A1221

Comment

Method

Meth Date : 27-Jan-1998 07:06 gar

Cal Date : 24,-JAN-1998 06:09

Als bottle: 1

Dil Factor: 1.000

Integrator: HP Genie

Target Version: 3.10
Client Smp ID: $200 \mathrm{ng} / \mathrm{ml}$ A1221

Inst ID: $9 \mathrm{c} 2 . \mathrm{i}$

\begin{tabular}{|c|c|c|c|c|c|c|c|}
\hline \multicolumn{8}{|c|}{ AMOUNTS } \\
\hline & & & & CAL-AMT & $\mathrm{ON}-\mathrm{COL}$ & & \\
\hline RT & EXP RT & DLT RT & RESPONSE & & ng) & TARGET RANGE & RATIO \\
\hline$=$ & $== \pm===$ & $=======$ & $=======$ & $== \pm==0$ & $==$ & 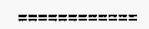 & $=====$ \\
\hline $23 \mathrm{Ar}$ & roclor-12 & & & & CAS \#: & $11104-28-2$ & \\
\hline 13.392 & 13.392 & 0.000 & 26063 & 0.20000 & 0.20000 & & $100.00(\mathrm{M})$ \\
\hline 13.751 & 13.751 & 0.000 & 24772 & 0.20000 & 0.20000 & $0.00-20.00$ & 95.05 \\
\hline 13.963 & 13.963 & 0.000 & 61872 & 0.20000 & 0.20000 & $0.00-20.00$ & 237.39 \\
\hline & & Average 0 & Amounts $=$ & & 0.20000 & & \\
\hline $29 \mathrm{Ts}$ & etrachlor & ro-m-xyle & & & CAS \#: & $877-09-8$ & \\
\hline 12.747 & 12.747 & 0.000 & 822351 & 0 & 0 & & 100.00 \\
\hline & & & & & & & \\
\hline $30 \mathrm{De}$ & ecachlorc & obiphenyl & & & CAS \#: & $2051-24-3$ & \\
\hline 43.055 & 43.055 & 0.000 & 437366 & 0 & 0 & & 100.00 \\
\hline
\end{tabular}

QC Flag Legend

M - Compound response manually integrated. 


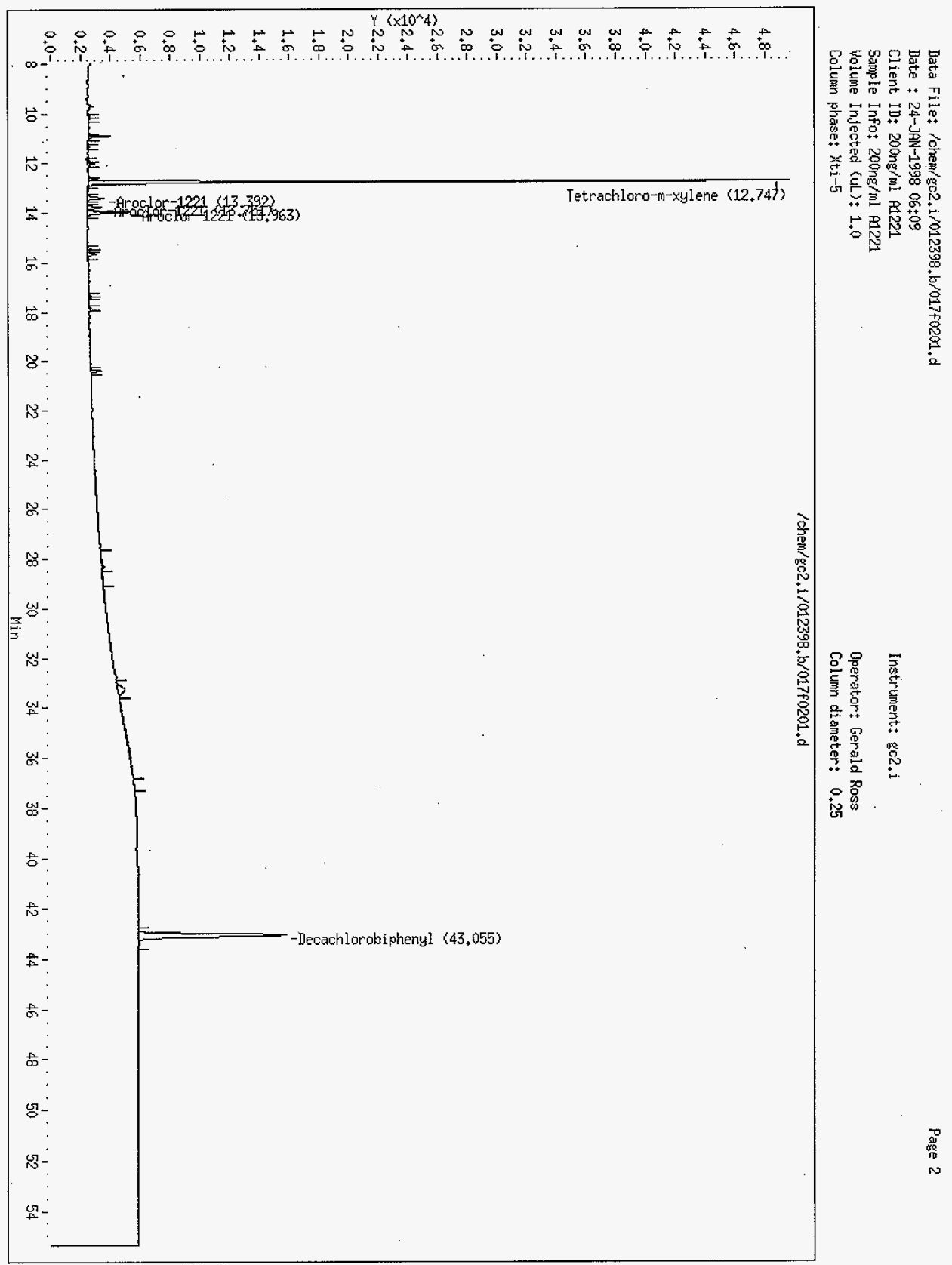


Data file : /chem/gc2.i/012398.b/018f0201.d

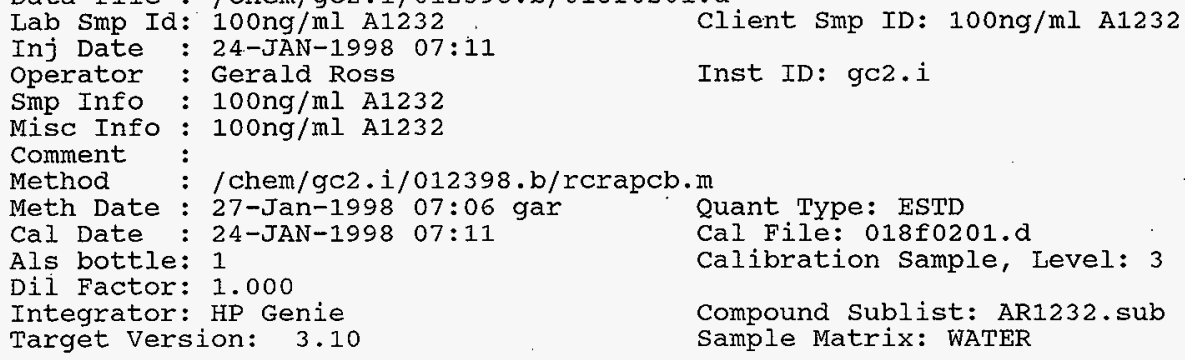

\begin{tabular}{|c|c|c|c|c|c|c|c|}
\hline \multirow{4}{*}{$\begin{array}{l}\text { RT } \\
==\end{array}$} & \multirow{4}{*}{$\begin{array}{l}\text { EXP RT } \\
=====\end{array}$} & \multirow[b]{3}{*}{ DLT RT } & \multicolumn{3}{|c|}{ AMOUNTS } & \multirow[b]{3}{*}{ TARGET RANGE } & \multirow{4}{*}{$\begin{array}{l}\text { RATI0 } \\
====\end{array}$} \\
\hline & & & \multicolumn{2}{|r|}{ CAL-AMT } & $\mathrm{ON}-\mathrm{COL}$ & & \\
\hline & & & RESPONSE & $n g)$ & ( $n g)$ & & \\
\hline & & = & $==== \pm= \pm=$ & =n=ニ=n= & $== \pm== \pm=$ & 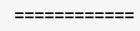 & \\
\hline \multicolumn{3}{|c|}{24 Aroclor -1232} & & \multicolumn{3}{|c|}{ CAS \#: $1114-16-5$} & \\
\hline 15.500 & 15.500 & $0 \quad 0.000$ & 987 & 0.10000 & 0.10000 & & $100.00(M)$ \\
\hline 17.320 & 17.320 & 0.000 & 1281 & 0.10000 & 0.10000 & $0.00-20.00$ & 129.79 \\
\hline 17.393 & 17.393 & $3 \quad 0.000$ & 1446 & 0.10000 & 0.10000 & $0.00-20.00$ & 146.50 \\
\hline \multicolumn{4}{|c|}{ Average of Peak Amounts } & & 0.10000 & & \\
\hline
\end{tabular}

\begin{tabular}{|c|c|c|c|c|c|c|}
\hline \multicolumn{4}{|c|}{29 Tetrachloro-m-xylene } & \multicolumn{3}{|c|}{ CAS \#: $877-09-8$} \\
\hline 12.753 & 12.753 & 0.000 & 34682 & 0 & 0 & 100.00 \\
\hline \multicolumn{4}{|c|}{30 Decachlorobiphenyl } & \multicolumn{3}{|c|}{ CAS \#: $2051-24-3$} \\
\hline 43.061 & 43.061 & 0.000 & 59836 & 0 & 0 & 100.00 \\
\hline
\end{tabular}

QC Flag Legend

M - Compound response manually integrated. 
Data File: /chem/gc2.i/012398.b/019f0201.d

Report Date: 27-Jan-1998 07:06

\section{2-s Laboratory}

Data file : /chem/gc2.i/012398.b/019f0201.a

Lab Smp Id: 100ng/ml A1242

Inj Date : 24-JAN-1998 08:14

Operator : Gerald Ross

Smp Info : $100 \mathrm{ng} / \mathrm{ml}$ A 1242

Misc Info: $100 \mathrm{ng} / \mathrm{ml} \mathrm{A} 1242$

Comment

Method : /chem/gc2.i/012398.b/rcrapcb.m

Meth Date : 27-Jan-1998 07:06 gar Quant Type: ESTD

Cal Date : 24-JAN-1998 08:14

Cal File: 019f0201.d

Als bottle: 1

Dil Factor: 1.000

Integrator: HP Genie

Target Version: 3.10

client Smp ID: 100ng/ml A1242

Inst ID: $g c 2 . i$

Calibration Sample, Level: 3

Compound sublist: AR1242. sub

Sample Matrix: WATER

\begin{tabular}{|c|c|c|c|c|c|c|c|}
\hline \multirow{4}{*}{$\begin{array}{l}\text { RT } \\
==\end{array}$} & \multirow{3}{*}{\multicolumn{2}{|c|}{ EXP RT DLT RT }} & \multicolumn{3}{|c|}{ AMOUNTS } & \multirow[b]{3}{*}{ TARGET RANGE } & \multirow[b]{3}{*}{ RATIO } \\
\hline & & & \multicolumn{2}{|r|}{ CAL-AMT } & $\mathrm{ON}-\mathrm{COL}$ & & \\
\hline & & & RESPONSE & ng) & ( $\quad n g)$ & & \\
\hline & $== \pm===$ & $==\mathbf{=}=\mathbf{=}=$ & $===== \pm= \pm$ & $\overline{m=}====$ & $======$ & $===========$ & $== \pm==$ \\
\hline \multicolumn{3}{|c|}{25 Aroclor -1242} & & \multicolumn{3}{|c|}{ CAS \#: $53469-21-9$} & \\
\hline 17.833 & 17.833 & 0.000 & 2731 & 0.10000 & 0.10000 & & $100.00(M)$ \\
\hline 20.379 & 20.379 & 0.000 & 6042 & 0.10000 & 0.10000 & $0.00-20.00$ & 221.24 \\
\hline 21.923 & 21.923 & 0.000 & 3078 & 0.10000 & 0.10000 & $0.00-20.00$ & 112.71 \\
\hline \multicolumn{4}{|c|}{ Average of Peak Amounts = } & \multicolumn{2}{|r|}{0.10000} & & \\
\hline \multicolumn{4}{|c|}{29 Tetrachloro-m-xylene } & \multirow{2}{*}{\multicolumn{3}{|c|}{$\begin{array}{c}\text { CAS \#: } 877-09-8 \\
0\end{array}$}} & \\
\hline 12.752 & 12.752 & 0.000 & 57609 & & & & 100.00 \\
\hline \multicolumn{3}{|c|}{30 Decachlorobiphenyl } & & \multirow{2}{*}{\multicolumn{3}{|c|}{$\begin{array}{c}\text { CAS \#: } 2051-24-3 \\
0\end{array}$}} & \\
\hline 43.060 & 43.060 & 0.000 & 95274 & & & & 100.00 \\
\hline
\end{tabular}

QC Flag Legend

M - Compound response manually integrated. 


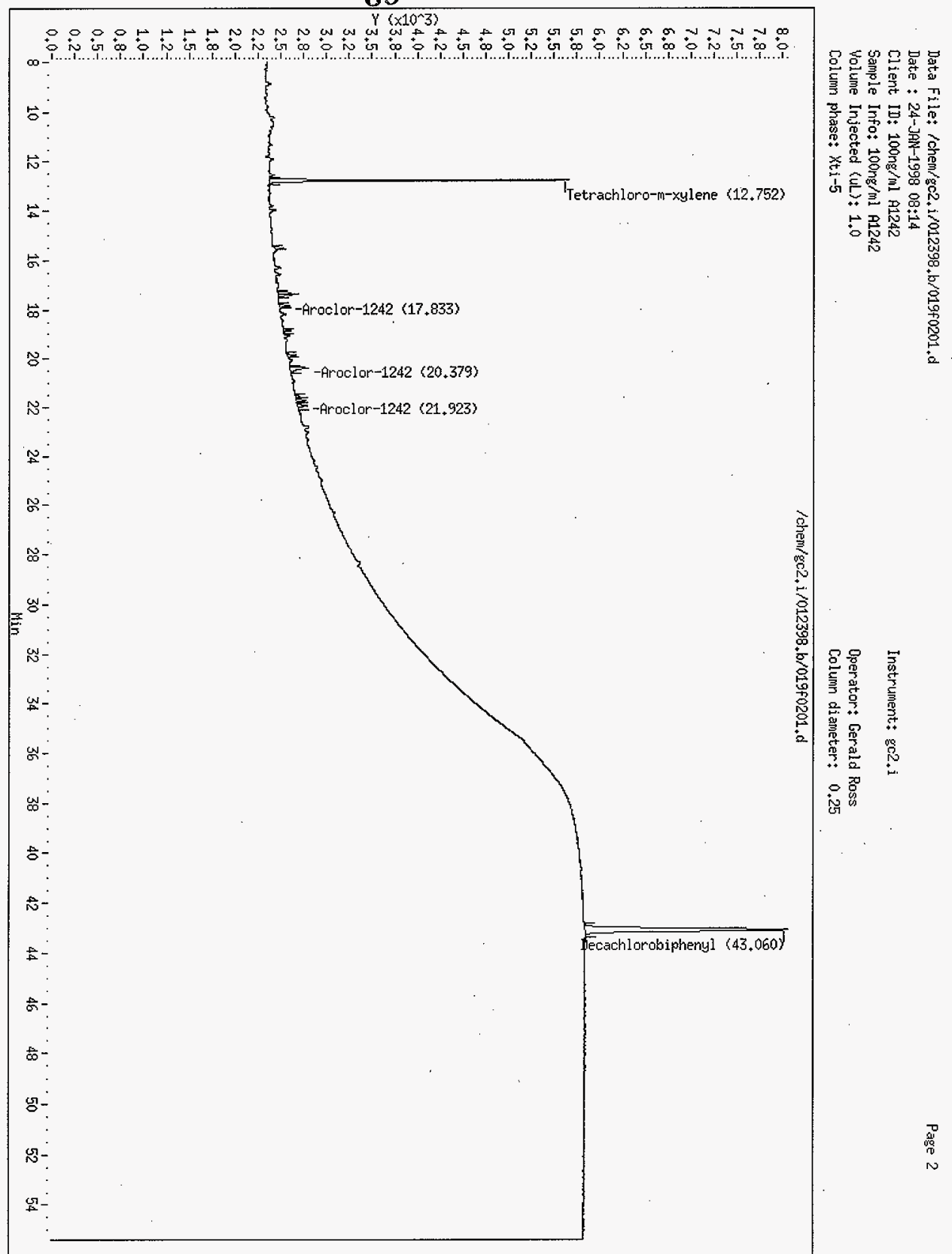


Data File: /chem/gc2.i/012398.b/020f0201.d

Report Date: 30-Jan-1998 11:53

Data file : /chem/gc2.i/012398.b/020f0201.d

Lab Smp Id: Inst.blnk

Inj Date : 24-JAN-1998 09:16

Operator : Gerald Ross

Smp Info : Inst.blnk

Misc Info: Inst.blnk

Comment

Method

/chem/gc2.i/012398.b/rcrapcb.m

Meth Date : 27-Jan-1998 07:18 gar

Cal Date : 24-JAN-1998 05:06

Als bottle: 1

Dil Factor: 1.000

Integrator: HP Genie

Target Version: 3.10
Client Smp ID: Inst.blnk

Inst ID: gc2.i
Quant Type: ESTD

Cal File: 016f0201.d

Compound Sublist: Aroclors.sub Sample Matrix: SOIL
CONCENTRATIONS ON-COL FINAL

RT EXP RT DLT RT

RESPONSE ( $\mathrm{ng}$ ) (ug/Kg) TARGET RANGE RATIO

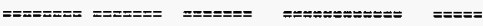

Peaks not detected for Quant. or Qual. signal(s).

Peaks not detected for Quant. or Qual. signal(s).

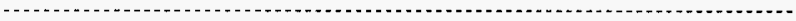

24 Aroclor -1232

CAS \#: $1114-16-5$

Peaks not detected for Quant. or Qual. signal(s).

25 Aroclor $1242 \quad$ CAS \#! 53469-21-9

Peaks not detected for Quant. or Qual. signal(s).

26 Aroclor -1248

CAS \#: $12672-29-6$

Peaks not detected for Quant. or Qual. signal(s).

Peaks not detected for Quant. or Qual. signal(s). 
Data File: /chem/gc2.i/012398.b/020f0201.d

Report Date: 30-Jan-1998 11:53

\section{HNF-SD-WA-DP-290 REV.O}

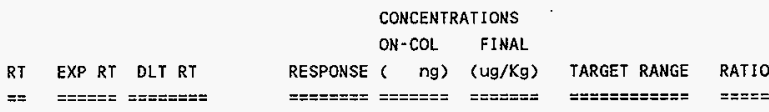

28 Aroclor -1260

CAS \#: $11096-82-5$

Peaks not detected for Quant. or Qual. signal(s).

\begin{tabular}{|c|c|c|c|c|c|c|}
\hline \multicolumn{4}{|c|}{$\$ 29$ Tetrachloro-m-xylene } & \multicolumn{3}{|c|}{ CAS \#: $877-09-8$} \\
\hline 12.749 & 12.750 & -0.001 & 607701 & 0 & 125.84 & 100.00 \\
\hline \multicolumn{4}{|c|}{$\$ 30$ Decach Lorobiphenyl } & \multicolumn{3}{|c|}{ CAS \#: $2051-24-3$} \\
\hline 43.059 & 43.059 & 0.000 & 358845 & 0 & 144.83 & 100.00 \\
\hline
\end{tabular}


Data File: /chem/gc2.i/012398.b/020f0201.d Report Date: 30-Jan-1998-11:53

\section{HNF-SD-WA-DP-290 REV. 0}

\section{2-S Iaboratory}

\section{RECOVERY REPORT}

Client Name:

Sample Matrix: SOLID

Client SDG: 012398

Lab Smp Id: Inst.blnk

Fraction: PEST

Level: LOW

Client Smp ID: Inst.blnk

Data Type: GC MULTI COMP

Operator: Gerald Ross

SpikeList File: RESOLUTION.spk

Method File: /chem/gc2.i/012398.b/rcrapcb.m

SampleType: SAMPLE

Quant Type: ESTD

Misc Info: Inst.blnk

\begin{tabular}{|c|c|c|c|c|}
\hline SURROGATE COMPOUND & $\begin{array}{l}\text { CONC } \\
\text { ADDED } \\
\mathrm{ug} / \mathrm{Kg}\end{array}$ & $\begin{array}{c}\text { CONC } \\
\text { RECOVERED } \\
\text { ug/Kg }\end{array}$ & RECOVERED & LIMITS \\
\hline $\begin{array}{lrl}\$ & 29 & \text { Tetrachloro-m-xyle } \\
\$ & 30 \text { Decachlorobiphenyl }\end{array}$ & $\begin{array}{l}104.00 \\
104.00\end{array}$ & $\begin{array}{l}125.84 \\
144.83\end{array}$ & $\begin{array}{l}121.00 \\
139.26\end{array}$ & $\begin{array}{l}20-150 \\
20-150\end{array}$ \\
\hline
\end{tabular}




\section{HNF-SD-WAADP-290 REV. 0}

Data File: /chem/gc2.i/012398.b/020f0201.d

Report Date: 30-Jan-1998 11:53

\section{2-s Laboratory \\ TARGET COMPOUNDS}

Client Name:

Lab Smp Id: Inst.blnk

Sample Location:

Sample Date:

Sample Matrix: SOIL

Analysis Type: PEST

Data Type: GC MULTI COMP

Misc Info: Inst.blnk
Client SDG: 012398

Client Smp ID: Inst.blnk

Sample Point:

Date Received:

Quant Type: ESTD

Level: Low

Operator: Gerald Ross

CAS NO.

COMPOUND

CONCENTRATION UNITS:

( $\mathrm{ug} / \mathrm{L}$ or $\mathrm{ug} / \mathrm{KG}$ ) $\mathrm{ug} / \mathrm{Kg}$

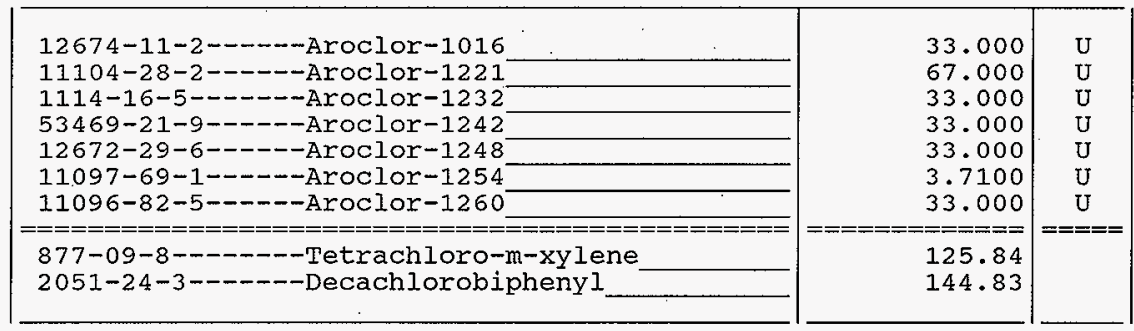


HNF-SD-WRA-DP-290 REV. 0

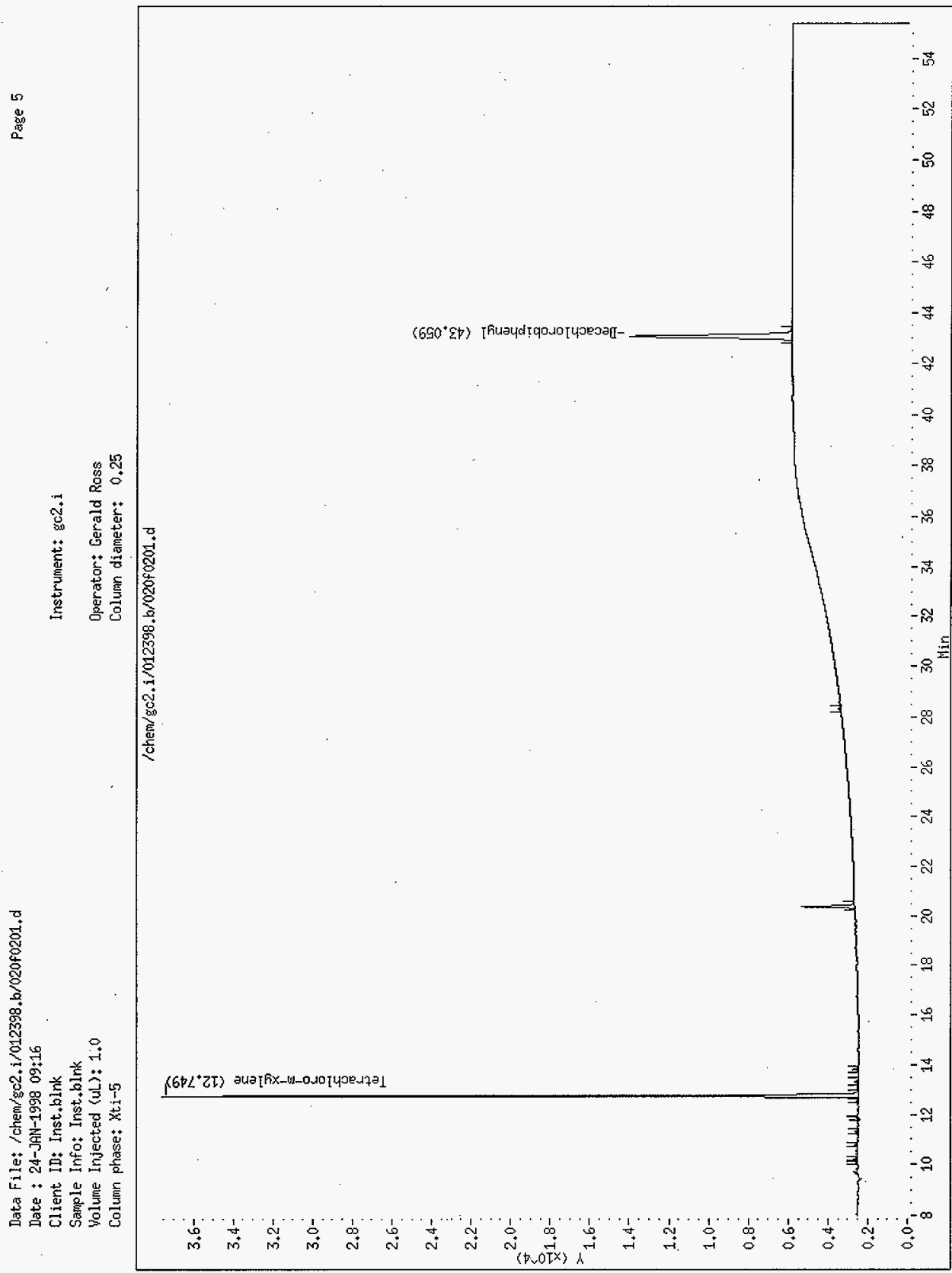




\section{HNF-SD-WA-DP-290 REV.O}

Data File:/chem/gc2.i/012398.b/021f0201.d

Report Date: 30-Jan-1998 11:53

Page 1

\section{2-S Laboratory}

Data file: /chem/gc2.i/012398,b/021f0201.d

Lab Smp Id: meth blnk 00935

Client Smp ID: meth blnk 00935

Inj Date : 24-JAN-1998 10:19

operator : Gerald Ross

Inst ID: $g \mathrm{c} 2 . \mathrm{i}$

Smp Info : meth blnk 00935

Misc Info : meth blnk 00935

Comment

Method

/chem/gc2.i/012398.b/rcrapcb.m

Meth Date : 27-Jan-1998 07:18 gar

Cal Date : 24-JAN-1998 05:06

Als bottle: 1

Dil Factor: 1.000

Integrator: HP Genie

Target Version: 3.10

Quant Type: ESTD

Cal File: 016f0201.d

QC Sample: BLANK

Compound Sublist: Aroclors.sub Sample Matrix: SOIL

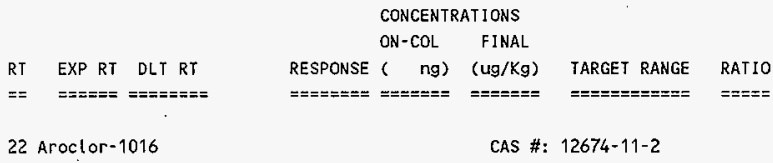

Peaks not detected for Quant. or Qual. signal(s).

23 Aroclor -1221

CAS \#: $11104-28-2$

Peaks not detected for Quant, or Qual. signal(s).

24 Aroctor -1232

CAS \#: $1114-16-5$

operator disabled compound identification.

25 Aroclor -1242

CAS \#: $53469-21-9$

Peaks not detected for Quant. or Qual. signal(s).

26 Aroclor -1248 CAS \#: 12672-29-6

Peaks not detected for Quant. or Qual. signal (s).

27 Aroclor -1254

CAS \#: $11097-69-1$

Peaks not detected for Quant. or Quat. signal(s). 


\section{HNF-SD-WAA-DP-290 REV. 0}

Data File: /chem/gc2.i/012398.b/021f0201.d Report Date: 30-Jan-1998 11:53

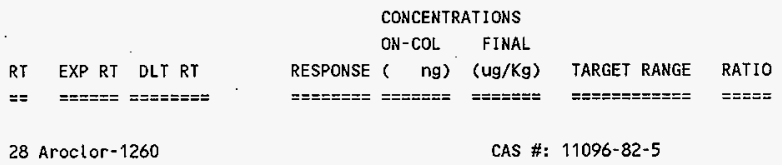

Operator disabled compound identification.

$\$ 29$ Tetrachloro-m-xylene

CAS \#: $877-09-8$

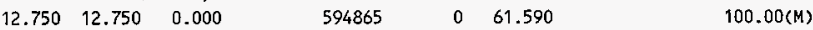

.

$\$ 30$ Decachlorobiphenyl

CAS \#: $2051-24-3$

$\begin{array}{lll}43.058 & 43.059 & -0.001\end{array}$

$\begin{array}{lll}534808 & 0.10792 \quad 107.92\end{array}$

100.00

QC Flag Legend

M - Compound response manually integrated. 
Report Date: 30-Jan-1998 11:53

$$
\text { 222-S Iaboratory }
$$

RECOVERY REPORT

Client Name:

Sample Matrix: SOLID

Lab Smp Id: meth bink 00935 Level: LOW

Data Type: GC MULTI COMP

Spikelist File: RESOLUTION.spk

Method File: /chem/gc2.i/012398.b/rcrapcb.m

Misc Info: meth blnk 00935
Client SDG: 012398

Fraction: PEST

Client Smp ID: meth blnk 00935 Operator: Gerald Ross

SampleType: BLANK

Quant Type: ESTD

\begin{tabular}{|c|c|c|c|c|}
\hline SURROGATE COMPOUND & $\begin{array}{l}\text { CONC } \\
\text { ADDED } \\
u q / K q\end{array}$ & $\begin{array}{c}\text { CONC } \\
\text { RECOVERED } \\
\text { U } / \mathrm{Kg}\end{array}$ & $\stackrel{\circ}{\%}$ RECOVERED & LIMITS \\
\hline $\begin{array}{lll}\$ & 29 & \text { Tetrachloro-m-xyle } \\
\$ & 30 & \text { Decachlorobiphenyl }\end{array}$ & $\begin{array}{l}100.00 \\
100.00\end{array}$ & $\begin{array}{l}61.590 \\
107.92\end{array}$ & $\begin{array}{r}61.59 \\
107.93\end{array}$ & $\begin{array}{l}20-150 \\
20-150\end{array}$ \\
\hline
\end{tabular}


Data File: /chem/gc2.i/012398.b/021f0201.d

\section{2-S Laboratory}

TARGET COMPOUNDS

client Name:

Lab Smp Id: meth blnk 00935

Sample Location:

Sample Date:

Sample Matrix: SoIL

Analysis Type: PEST

Data Type: GC MULTI COMP

Misc Info: meth blnk 00935
Client SDG: 012398

Client Smp ID: meth blnk 00935

Sample Point:

Date Received:

Quant Type: ESTD

Level: LOW

Operator: Gerald Ross

CONCENTRATION UNITS:

CAS NO.

COMPOUND

(ug/L or $\mathrm{ug} / \mathrm{KG}$ ) ug/Kg

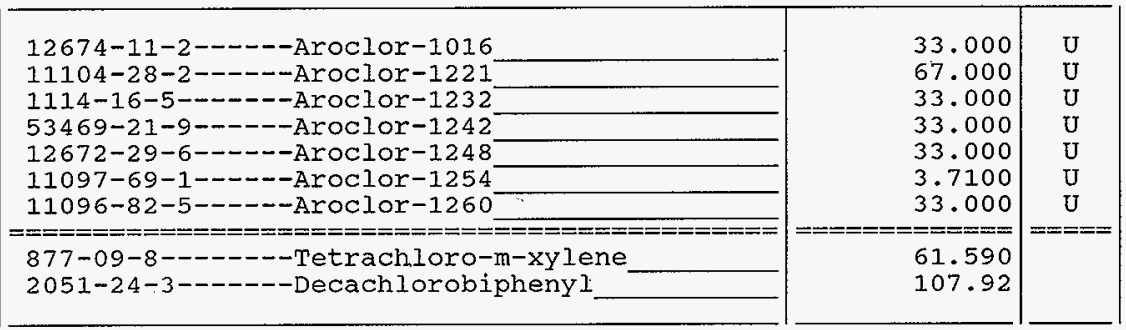




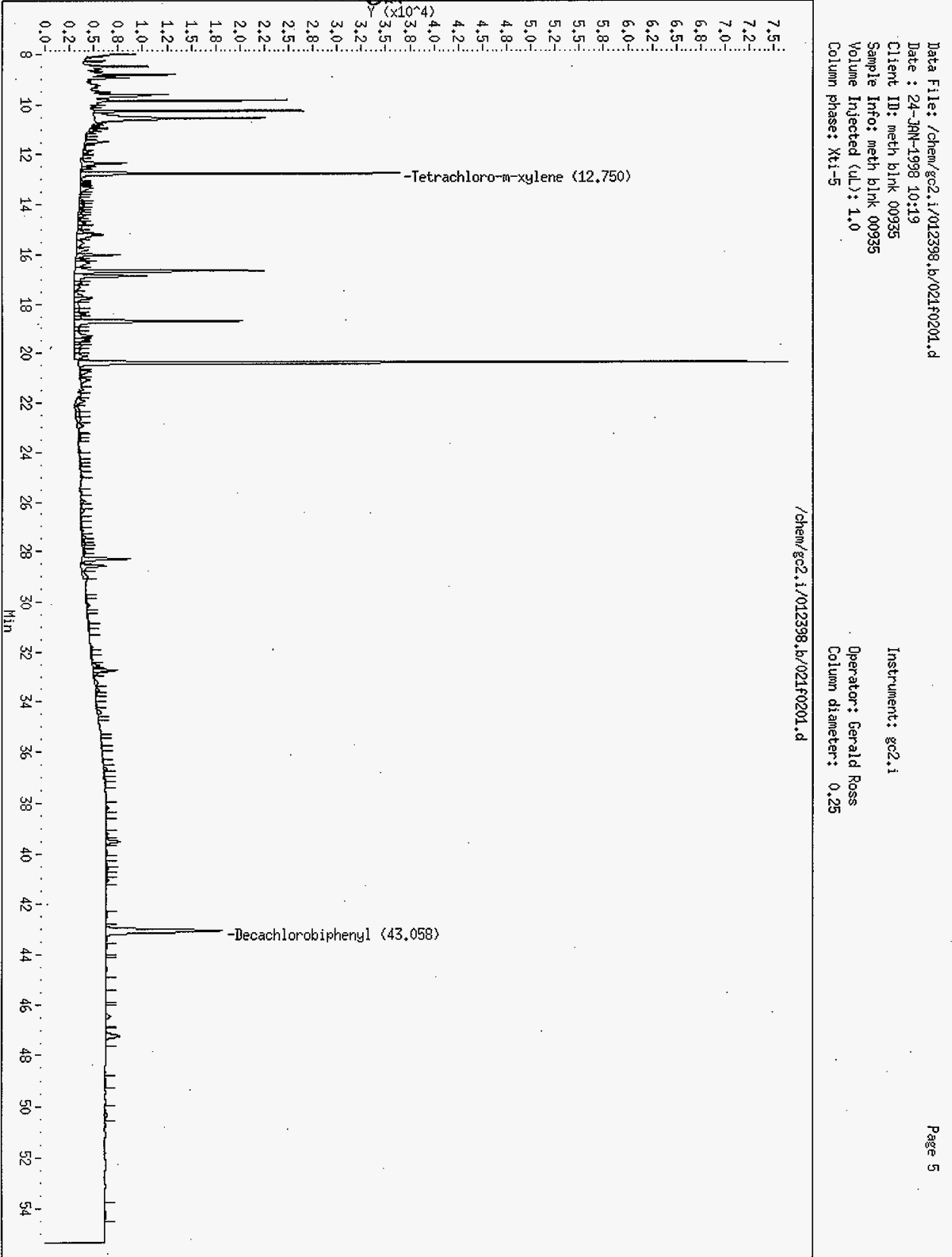




\section{HNF-SD-WMA-DP-290 REV. 0}

Data File: /chem/gc2.i/012398.b/022f0201.d

Page 1

Report Date: 30-Jan-1998 11:53

\section{2-S Laboratory}

Data file: /chem/gc2.i/012398.b/022f0201.d

Lab Smp Id: LCS spk 00937

Inj Date : 24-JAN-1998 11:22

Operator : Gerald Ross

Simp Info : LCS spk 00937

Client Smp ID: LCS spk 00937

Misc Info : 000000937

Comment

Method

/chem/gc2.i/012398.b/rcrapcb.m

Meth Date : 27-Jan-1998 07:18 gar

Cal Date : 24-JAN-1998 05:06

Quant Type: ESTD

Als bottle: 1

Dil. Factor: 1.000

Integrator: HP Genie

Target Version: 3.10

Inst ID: gc2.i

\begin{tabular}{|c|c|c|c|c|c|c|c|}
\hline & & & & CONCENTR & TIONS & & \\
\hline & & & & $\mathrm{ON}-\mathrm{COL}$ & FINAL & & \\
\hline RT & EXP RT & DLT RT & RESPONSE & ng) & (ug/Kg) & TARGET RANGE & RATIO \\
\hline$==$ & $======$ & $=== \pm====$ & $= \pm==\geq= \pm \Sigma=$ & 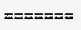 & $======$ & 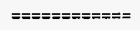 & $=== \pm=$ \\
\hline $22 \mathrm{Ar}$ & roclor-10 & 16 & & & CAS \#: & $12674-11-2$ & \\
\hline 15.502 & 15.500 & 0.002 & 56957 & 0.15178 & 151.78 & & 100.00 \\
\hline 17.305 & 17.311 & -0.006 & 29579 & 0 & 65.940 & $0.00-20.00$ & 51.93 \\
\hline 17.386 & 17.389 & -0.003 & 33652 & 0 & .60 .990 & $0.00-20.00$ & 59.08 \\
\hline & & Average 0 & oncentra & tion & 92.902 & & \\
\hline
\end{tabular}

28 Aroclor -1260

$26.211 \quad 26.212 \quad-0.001$

$27.443 \quad 27.448-0.005$

$29.004 \quad 29.007 \quad-0.003$

Average of Peak Concentrations =
CAS \#: $11096-82-5$

$\begin{array}{rrrrrr}55628 & 0.10216 & 102.16 & & & 100.00 \\ 51702 & 0 & 94.464 & 0.00- & 20.00 & 92.94 \\ 38404 & 0 & 78.878 & 0.00- & 20.00 & 69.04\end{array}$

CAS \#: $877-09-8$

Compound Sublist: Aroclors.sub Sample Matrix: SOIL

$\$ 29$ Tetrachtoro-m-xylene 52.934 $100.00(M)$

$\begin{array}{lll}12.747 & 12.750 & -0.003\end{array}$ 511263

CAS \#: $2051-24-3$

$\$ 30$ Decachlorobiphenyl

$\begin{array}{llll}43.058 & 43.059 & -0.001\end{array}$

\section{QC Flag Legend}

M - Compound response manually integrated. 


\section{HNF-SD-WM-DP-290 REV. 0}

Data File: /chem/gc2.i/012398.b/022f0201.d

Report Date: 30-Jan-1998 11:53

\section{2-S Laboratory \\ RECOVERY REPORT}

Client Name:

Sample Matrix: SOLID

Client SDG: 012398

Lab Smp Id: LCS spk 00937

Fraction: PEST

Level: LoW

Client Smp ID: LCS spk 00937

Data Type: GC MULTI COMP

Operator: Gerald Ross

Spikelist File: RESOLUTION.spk

SampleType: SAMPLE

Method File: / chem/gc2.i/012398.b/rcrapcb.m

Misc Info: 000000937

\begin{tabular}{|c|c|c|c|c|}
\hline SURROGATE COMPOUND & $\begin{array}{l}\text { CONC } \\
\text { ADDED } \\
\mathrm{ug} / \mathrm{Kg}\end{array}$ & $\begin{array}{c}\text { CONC } \\
\text { RECOVERED } \\
u g / \mathrm{Kg}\end{array}$ & $\stackrel{\frac{\%}{6}}{\text { RECOVERED }}$ & IIMITS \\
\hline $\begin{array}{lll}\$ & 29 & \text { Tetrachloro-m-xyle } \\
\$ & 30 \text { Decachlorobiphenyl }\end{array}$ & $\begin{array}{l}100.00 \\
100.00\end{array}$ & $\begin{array}{l}52.934 \\
69.212\end{array}$ & $\begin{array}{l}52.93 \\
69.21\end{array}$ & $\begin{array}{l}20-150 \\
20-150\end{array}$ \\
\hline
\end{tabular}




\section{HNF-SD-WAA-DP-290 REV. 0}

Data File: /chem/gc2.i/012398.b/022f0201.d

Report Date: 30-Jan-1998 11:53

\section{2-S Laboratory \\ TARGET COMPOUNDS}

Client Name:

Lab Smp Id: LCS spk 00937

Sample Location:

Sample Date:

Sample Matrix: SOII

Analysis Type: PEST

Data Type: GC MULTI COMP

Misc Info: 000000937
Client SDG: 012398

Client Smp ID: LCS spk 00937

Sample Point:

Date Received:

Quant Type: ESTD

Level: LoW

Operator: Gerald Ross

CAS NO.

COMPOUND

CONCENTRATION UNITS:

( $\mathrm{ug} / \mathrm{L}$ or $\mathrm{ug} / \mathrm{KG}$ ) $\mathrm{ug} / \mathrm{Kg}$

\begin{tabular}{|c|c|c|}
\hline $\begin{array}{l}12674-11-2----- \text { Aroclor }-1016 \\
11104-28-2----- \text { Aroclor }-1221 \\
1114-16-5---- \text { Aroclor }-1232 \\
53469-21-9---- \text { Aroclor }-1242 \\
12672-29-6----- \text { Aroclor }-1248 \\
11097-69-1---- \text { Aroclor }-1254 \\
11096-82-5---- \text { Aroclor }-1260\end{array}$ & $\begin{array}{r}92.902 \\
67.000 \\
33.000 \\
33.000 \\
33.000 \\
3.7100 \\
91.835\end{array}$ & $\begin{array}{l}U \\
U \\
U \\
U \\
U\end{array}$ \\
\hline $\begin{array}{l}877-09-8---1--T e t r a c h l o r o-m-x y l e n e \\
2051-24-3-\cdots-m-D e c a c h l o r o b i p h e n y l\end{array}$ & $\begin{array}{l}52.934 \\
69.212\end{array}$ & \\
\hline
\end{tabular}




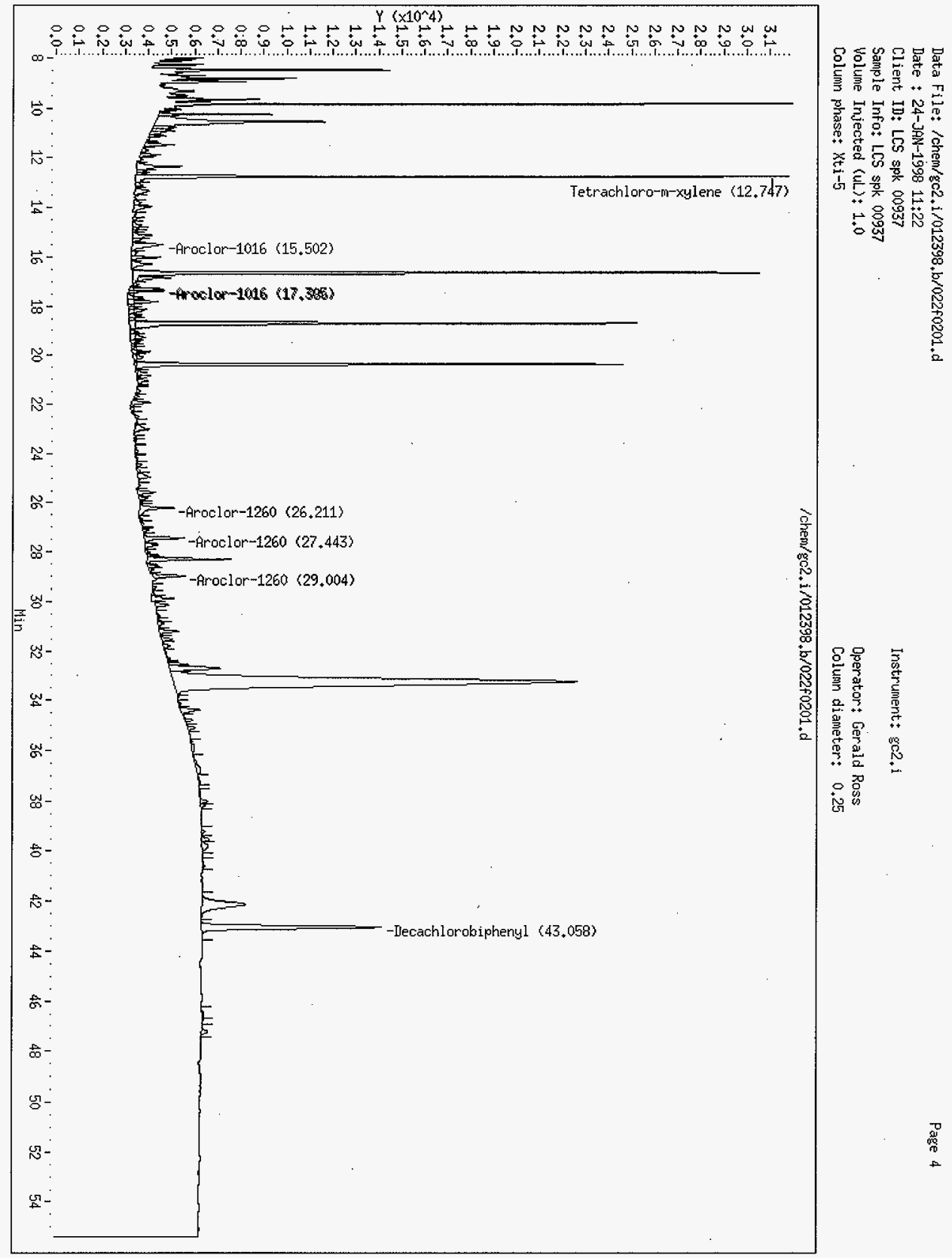


Data File:/chem/gc2.i/012398.b/023f0201.d

Page 1

Report Date: 30-Jan-1998 13:45

\author{
222-S Laboratory
}

Data file:/chem/gc2.i/012398.b/023f0201.d
Lab Smp Id: s97500235
Inj Date : 24-JAN-1998 12:24
Operator : Gerald Ross
Smp Info : 975023500927
Misc Info : $97 \mathrm{~S} 023500927$
comment :
Method : /chem/gc2.i/012398.b/rcrapcb.m
Meth Date : 27-Jan-1998 07:18 gar Quant Type: ESTD
Cal Date : 24-JAN-1998 05:06 Cal File: 016f0201.d
Als bottle: 1
Dil Factor: 10.000
Integrator: HP Genie
Target Version: 3.10
client Smp ID: S97S00235
Inst ID: $9 c 2 . i$
Compound Sublist: Aroclors.sub Sample Matrix: SOIL

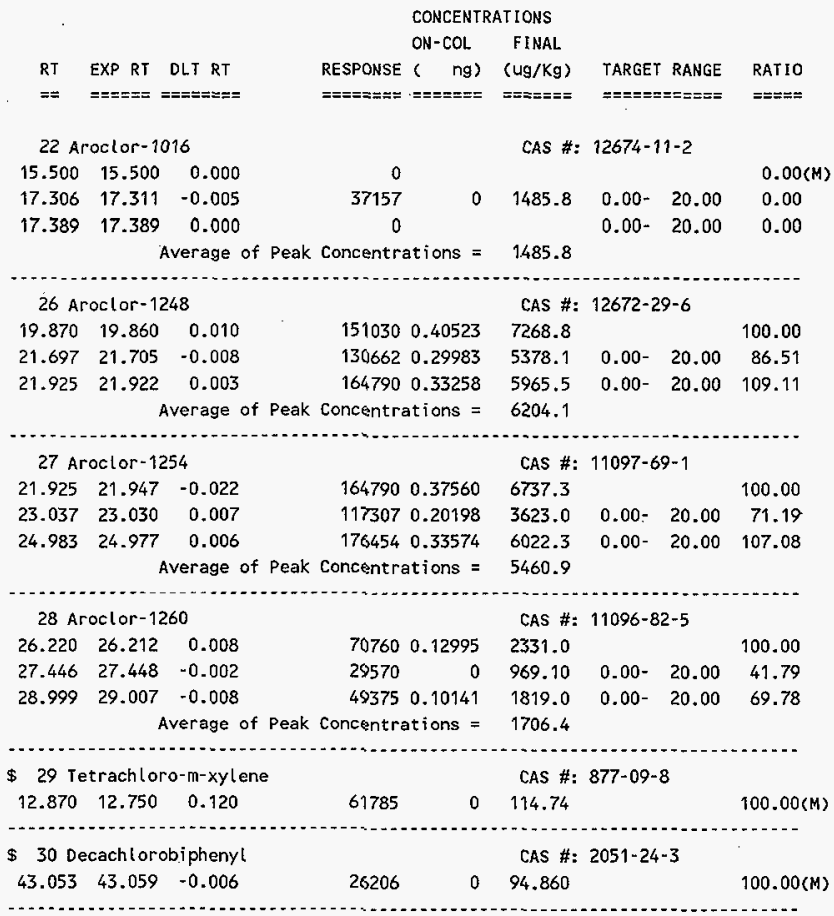


HNF-SD-WAADP-290 REV. 0

Data File: /chem/gc2.i/012398.b/023f0201.d

Report Date: 30-Jan-1998 13:45

QC Flag Legend

M - Compound response manually integrated. 


\section{2-S Laboratory}

\section{RECOVERY REPORT}

Client Name:

Sample Matrix: SOLID

Lab Smp Id: S97S00235

Level: LOW

Data Type: GC MULTI COMP

Spikelist File: Aroclor-1660.spk

Method $F i$ ie: /chem/gc2.i/01

Method File: /chem/gc2.i/012398.b/rcrapcb.m
Misc Info: $97 \mathrm{~S} 023500927$
Client SDG: 012398

Fraction: PEST

Client Smp ID: S97S00235

Operator: Gerald Ross

SampleType: SAMPLE

Quant Type: ESTD

\begin{tabular}{|c|c|c|c|c|}
\hline SURROGATE COMPOUND & $\begin{array}{l}\text { CONC } \\
\text { ADDED } \\
\mathrm{ug} / \mathrm{Kg}\end{array}$ & $\begin{array}{c}\text { CONC } \\
\text { RECOVERED } \\
\text { ug/Kg }\end{array}$ & $\begin{array}{c}\% \\
\text { RECOVERED }\end{array}$ & IIMITS \\
\hline $\begin{array}{l}\$ 29 \text { Tetrachloro-m-xyle } \\
\$ 30 \text { Decachlorobiphenyl }\end{array}$ & $\begin{array}{l}179.37 \\
179.37\end{array}$ & $\begin{array}{l}114.74 \\
94.860\end{array}$ & $\begin{array}{l}63.97 \\
52.88\end{array}$ & $\begin{array}{l}\overline{20-150} \\
20-150\end{array}$ \\
\hline
\end{tabular}




\section{2-S Laboratory \\ TARGET COMPOUNDS}

Client Name:

Lab Smp Id: S97500235

Sample Location:

Sample Date:

Sample Matrix: SoIL

Analysis Type: PEST

Data Type: GC MULTI COMP

Misc Info: $97 \mathrm{~S} 023500927$
Client SDG: 012398

Client Smp ID: $\$ 97 \$ 00235$

Sample Point:

Date Received:

Quant Type: ESTD

Level: LOW

Operator: Gerald Ross

CONCENTRATION UNITS :

( $\mathrm{ug} / \mathrm{L}$ or $\mathrm{ug} / \mathrm{KG}) \mathrm{ug} / \mathrm{Kg}$

\begin{tabular}{|c|}
\hline 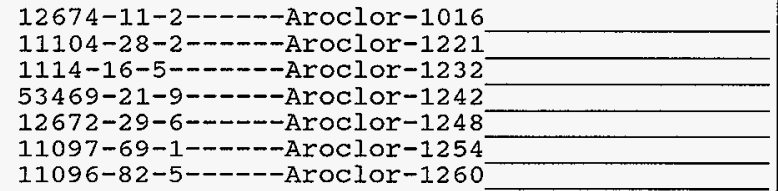 \\
\hline 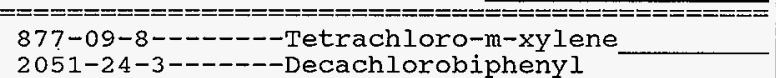 \\
\hline
\end{tabular}

\begin{tabular}{r|r|}
1485.8 & \\
670.00 & $\mathrm{U}$ \\
330.00 & $\mathrm{U}$ \\
330.00 & $\mathrm{U}$ \\
6204.1 & \\
5460.9 & \\
1706.4 & \\
114.74 \\
94.860
\end{tabular} \mid$===$




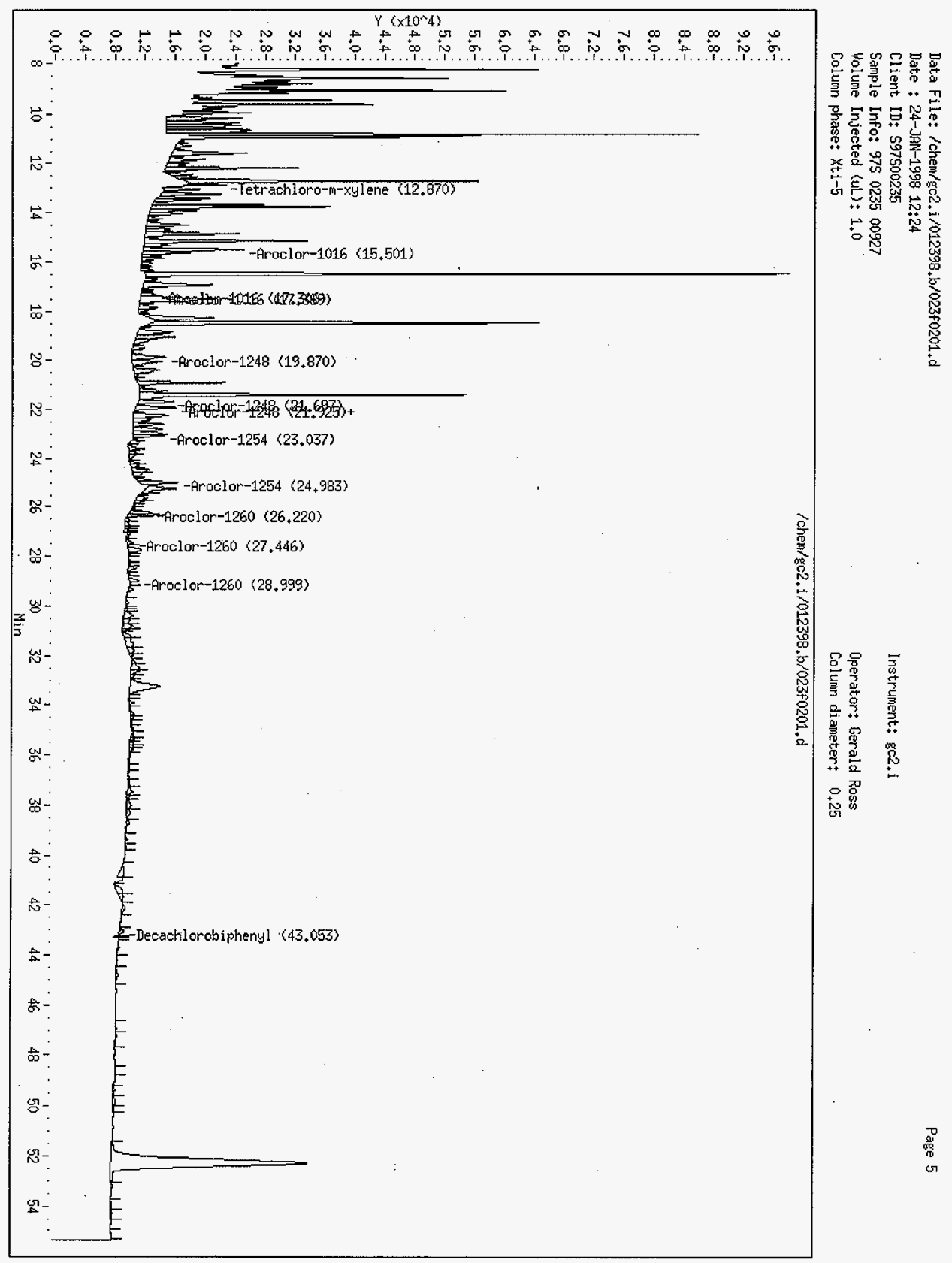


Data file : /chem/gc2.i/012398.b/024f0201.d

Lab Smp Id: inst. blnk

Inj Date : 24-JAN-1998 13:27

operator : Gerald Ross

Smp Info : inst. blnk

Misc Info : inst. blnk

Comment

Method

Meth Date

Cal Date

Als bottle: 1

Dil Factor: 1.000

Integrator: HP Genie

Target Version: 3.10

24-JAN-1998 05:06 client Smp ID: inst. blnk

Inst ID: $g c 2 . i$ /chem/gc2.i/012398.b/rcrapcb.m 27-Jan-1998 07:18 gar

Quant Type: ESTD

Cal File: 016f0201.d

Compound Sublist: Aroclors.sub Sample Matrix: SOIL

CONCENTRATIONS

ON-COL FINAL

RT EXP RT DLT RT RESPONSE ( $\mathrm{ng}$ ) (ug/Kg) TARGET RANGE RATIO

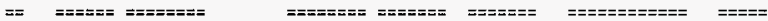

22 Aroclor-1016

CAS \#: $12674-11-2$

Peaks not detected for Quant. or Qual. signal(s).

23 Aroclor -1221

CAS \#: $11104-28-2$

Peaks not detected for Quant. or Qual. signal(s).

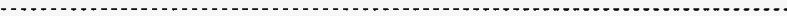

24 Aroclor -1232

CAS \#: $1114-16-5$

Peaks not detected for Quant. or Qual. signal(s).

25 Aroclor -1242

CAS \#: 53469-21-9

Peaks not detected for Quant. or Qual. signal(s).

26 Aroclor -1248

CAS \#: $12672-29-6$

Peaks not detected for Quant. or Qual. signal(s).

27 Aroclor -1254

CAS \#: $11097-69-1$

Peaks not detected for Quant. or Qual. signal(s). 
HNF-SD-WNADP-290 REV. 0

Data File: /chem/gc2.i/012398.b/024f0201.d

Report Date: 30-Jan-1998 11:53

\begin{tabular}{|c|c|c|c|c|c|c|}
\hline \multirow[b]{3}{*}{$R T$} & & \multicolumn{3}{|c|}{ CONCENTRATIONS } & \multirow[b]{3}{*}{ TARGET RANGE } & \multirow[b]{3}{*}{ RATIO } \\
\hline & & & $\mathrm{ON}-\mathrm{COL}$ & FINAL & & \\
\hline & EXP RT DLT RT & RESPONSE & ( $\mathrm{ng})$ & $(\mathrm{ug} / \mathrm{Kg})$ & & \\
\hline$==$ & 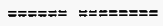 & $===-===$ & $== \pm=ニ==$ & $==\pi==\Xi=$ & $==\pi= \pm=\pi====$ & $====+$ \\
\hline
\end{tabular}

Peaks not detected for Quant. or Qual. signal(s).

$\$ 29$ Tetrachloro-m-xylene

CAS \#: $877-09-8$

$12.749 \quad 12.750 \quad-0.001$

526602

$0 \quad 109.04$

100.00

$\$ 30$ Decachlorobiphenyl

$\begin{array}{lll}43.060 & 43.059 \quad 0.001\end{array}$

CAS \#: 2051-24-3

$43.060 .43 .059-0.001$

$18200100 \quad 73.456$

100.00 


\section{HNF-SD-WRA-DP-290 REV. 0}

Data File: /chem/gc2.i/012398.b/024f0201.d

Report Date: 30-Jan-1998 11:53

\section{2-S Laboratory \\ RECOVERY REPORT}

Client Name:

Sample Matrix: SOLID

Lab Smp Id: inst. blnk

Level: LoW

Data Type: GC MULTI COMP

SpikeIist File: RESOLUTION.spk

Method File: / chem/gc2.i/012398.b/rcrapcb.m

Misc Info: inst. bInk
Client SDG: 012398

Fraction: PEST

Client Smp ID: inst. blnk

Operator: Gerald Ross

SampleType: SAMPLE

Quant Type: ESTD

\begin{tabular}{|c|c|c|c|c|}
\hline SURROGATE COMPOUND & $\begin{array}{l}\text { CONC } \\
\text { ADDED } \\
u g / K g\end{array}$ & $\begin{array}{c}\text { CONC } \\
\text { RECOVERED } \\
\text { ug/Kg }\end{array}$ & RECOVERED & LIMITS \\
\hline $\begin{array}{lll}\$ & 29 & \text { Tetrachloro-m-xyle } \\
\$ & 30 \text { Decachlorobiphenyl }\end{array}$ & $\begin{array}{l}104.00 \\
104.00\end{array}$ & $\begin{array}{l}109.04 \\
73.456\end{array}$ & $\begin{array}{r}104.85 \\
70.63\end{array}$ & $\begin{array}{l}20-150 \\
20-150\end{array}$ \\
\hline
\end{tabular}




\section{2-S Laboratory \\ TARGET COMPOUNDS}

Client Name:

Lab Smp Id: inst. blnk

Sample Location:

Sample Date:

Sample Matrix: SoIL

Analysis Type: PEST

Data Type: GC MULTI COMP

Misc Info: inst. blnk
Client SDG: 012398

Client Smp ID: inst. blnk

Sample Point:

Date Received:

Quant Type: ESTD

Level: LOW

operator: Gerald Ross

CONCENTRATION UNITS:

(ug/L or $\mathrm{ug} / \mathrm{KG}$ ) ug/Kg

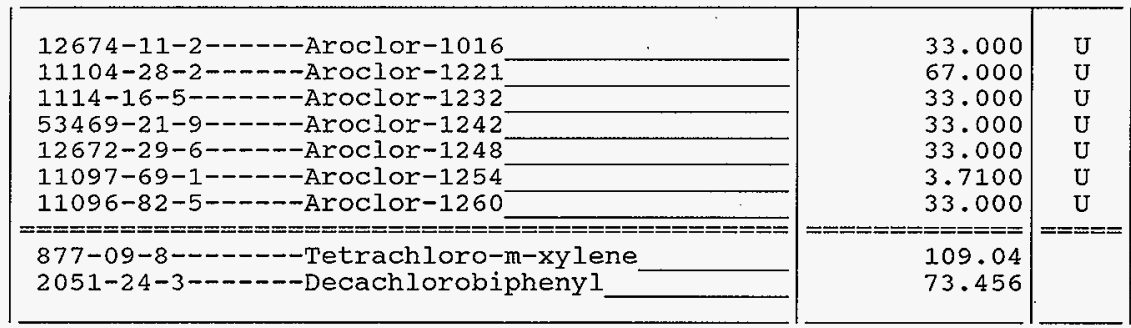




\section{HNF-SD-WA-DP-290 REV. 0}

Data File: /chem/gc2.i/012398.b/025f0201.d

Report Date: 30-Jan-1998 13:45

Page 1

\section{2-S Laboratory}

Data file : /chem/gc2.i/012398.b/025f0201.d

Lab Smp Id: 97S 0235D00929

Inj Date : 24-JAN-1998 14:29

operator : Gerald Ross

Smp Info : 97S 0235D00929

Misc Info: $97 \mathrm{~S}$ 0235D00929

comment :

Method : /chem/gc2.i/012398.b/rcrapcb.m

Meth Date : 27-Jan-1998 07:18 gar Quant Type: ESTD

Cal Date : 24-JAN-1998 05:06 Cal File: 016f0201.d

Als bottle: 1

Dil Factor: 10.000

Integrator: HP Genie

Target Version: 3.10
Client Smp ID: 97S 0235D00929

Inst ID: $g c 2 . i$
Compound Sublist: Aroclors. sub Sample Matrix: SoIL

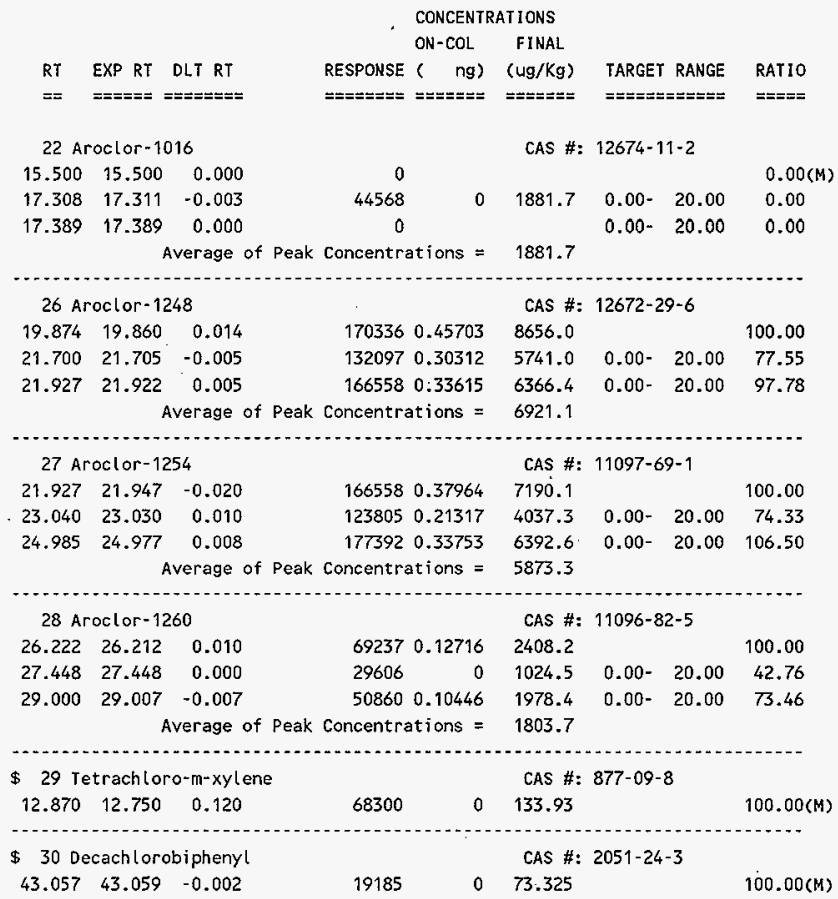


Data File: /chem/gc2.i./012398.b/025f0201.d

Report Date: 30-Jan-1998 13:45

QC Flag Legend

M - Compound response manually integrated. 
HNF-SD-WAA-DP-290 REV. 0

Data File: /chem/gc2.i/012398.b/025f0201.d

Report Date: 30-Jan-1998 13:45

$$
\text { 222-S Laboratory }
$$

\section{RECOVERY REPORT}

Client Name:

Sample Matrix: SOLID

Lab Smp Id: 97S 0235D00929

Level: Low

Data Type: GC MULTI COMP

SpikeList File: RESOLUTION.spk

Method File:/chem/gc2.i/012398.b/rorapcb.m

Misc Info: 97S 0235D00929
Client SDG: 012398

Fraction: PEST

Client Smp ID: 97S 0235D00929

Operator: Gerald Ross

SampleType: SAMPLE

Quant Type: ESTD

\begin{tabular}{|c|c|c|c|c|}
\hline SURROGATE COMPOUND & $\begin{array}{l}\text { CONC } \\
\text { ADDED }\end{array}$ & $\begin{array}{c}\text { CONC } \\
\text { RECOVERED }\end{array}$ & $\stackrel{\%}{\circ}$ RECOVERED & LIMITS \\
\hline $\begin{array}{lll}\$ & 29 & \text { Tetrachloro-m-xyle } \\
\$ & 30 & \text { Decachlorobiphenyl }\end{array}$ & $\begin{array}{l}189.39 \\
189.39\end{array}$ & $\begin{array}{l}133.93 \\
73.325\end{array}$ & $\begin{array}{l}70.71 \\
38.72\end{array}$ & $\begin{array}{l}20-150 \\
20-150\end{array}$ \\
\hline
\end{tabular}


HNF-SD-WM-DP-290 REV. 0

Data File: /chem/gc2.i/012398.b/025f0201.d

Report Date: 30-Jan-1998 13:45

\section{2-S Laboratory \\ TARGET COMPOUNDS}

Client Name:

Lab Smp Id: 97S 0235D00929

Sample Location:

Sample Date:

Sample Matrix: SOIL

Analysis Type: PEST

Data Type: GC MULTI COMP

Misc Info: $97 \mathrm{~S}$ 0235D00929
Client SDG: 012398

Client Smp ID: 97S 0235D00929

Sample Point:

Date Received:

Quant Type: ESTD

Level: LOW

Operator: Gerald Ross

CAS NO.

COMPOUND

CONCENTRATION UNITS:

( $u g / L$ or $u g / K G$ ) ug/Kg

12674-11-2------Aroclor-1016

$11104-28-2------$ Aroclor -1221

1114-16-5-------Aroclor -1232

53469-21-9----Aroclor -1242

12672-29-6------Aroclor -1248

11097-69-1------Aroclor -1254

11096-82-5----Aroclor-1260

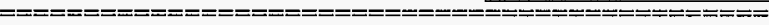
877-09-8------Tetrachloro-m-xylene 2051-24-3------Decachlorobiphenyl.

\begin{tabular}{|c|c|}
\hline $\begin{array}{l}1881.7 \\
670.00 \\
330.00 \\
330.00 \\
6921.1 \\
5873.3 \\
1803.7\end{array}$ & $\begin{array}{l}U \\
U \\
U\end{array}$ \\
\hline $\begin{aligned}===== & ====== \\
& 133.93 \\
& 73.325\end{aligned}$ & $====$ \\
\hline
\end{tabular}




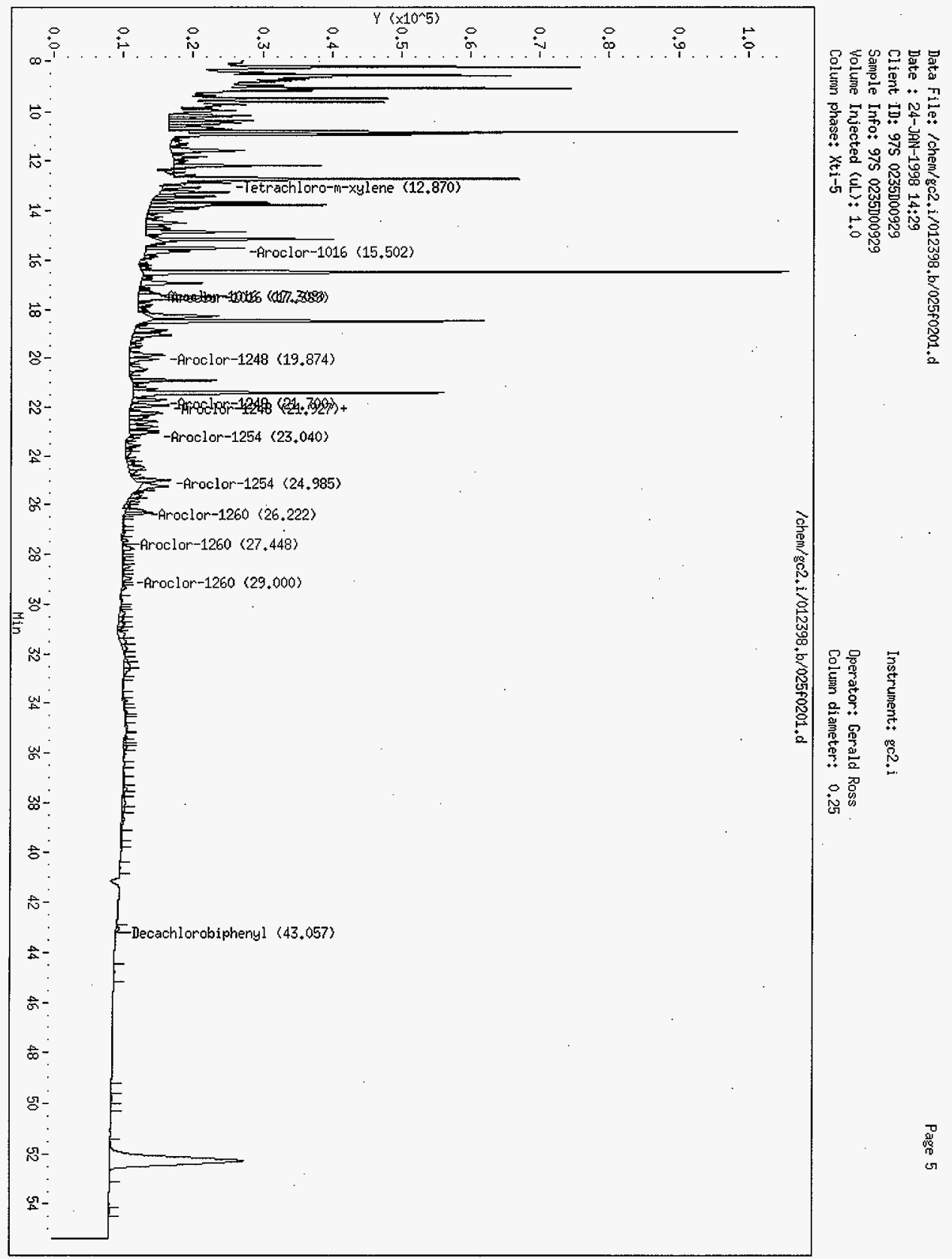


Data file : /chem/gc2.i/012398.b/026f0201.d

Lab smp Id: S97500235MS

Inj Date : 24-JAN-1998 15:32

Operator : Gerald Ross

Smp Info : 97S 0235MS00931

Misc Info : 975 0235MS00931

Comment

Method

/chem/gc2.i/012398.b/rcrapcb.m

Meth Date : 27-Jan-1998 07:18 gar

Cal Date : 24-JAN-1998 05:06

Als bottle: 1

Dil Factor: 10.000

Integrator: HP Genie

Target Version: 3.10
Client Smp ID: S97500235MS

Inst ID: $g c 2 \cdot i$
Quant Type: ESTD

Cal File: 016f0201.d

QC Sample: MS

Compound Sublist: Aroclors.sub Sample Matrix: SOIL

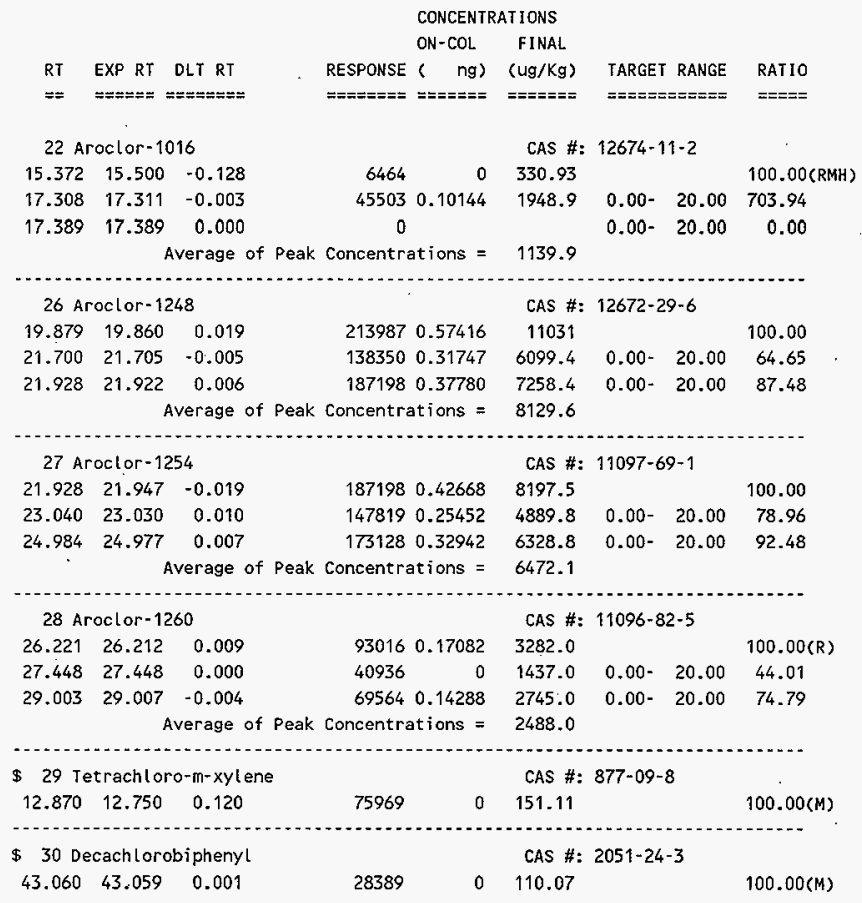


HNF-SD-WHADP-290 REV. 0

Data File:/chem/gc2.i/012398.b/026f0201.d Report Date: 30-Jan-1998 13:45

QC Flag Legend

$R$ - Spike/Surrogate failed recovery limits.

M - Compound response manually integrated.

H - Operator selected an alternate compound hit. 
Data File:/chem/gc2.i/012398.b/026f0201.d

Report Date: 30-Jan-1998 13:45

\section{2-S Laboratory}

\section{RECOVERY REPORT}

ciient Name:

Sample Matrix: SoLID

Lab Smp Id: S97500235MS

Level: LOW

Data Type: GC MULTI COMP

Spikelist File: Aroclor-1660.spk

Method File: /chem/gc2.i/012398.b/rcrapcb.m
Client SDG: 012398

Fraction: PEST

Client Smp ID: S97S00235MS

operator: Gerald Ross

SampleType: MS

Quant Type: ESTD

Misc Info: 97S 0235MS00931

\begin{tabular}{|c|c|c|c|c|}
\hline SPIKE COMPOUND & $\begin{array}{l}\text { CONC } \\
\text { ADDED } \\
\mathrm{ug} / \mathrm{Kg}\end{array}$ & $\begin{array}{c}\text { CONC } \\
\text { RECOVERED } \\
\text { ug/Kg }\end{array}$ & $\begin{array}{c}\% \\
\text { RECOVERED }\end{array}$ & LIMITS \\
\hline $\begin{array}{ll}22 & \text { Aroclor-1016 } \\
28 & \text { Aroclor }-1260\end{array}$ & $\begin{array}{l}192.12 \\
192.12\end{array}$ & $\begin{array}{l}1139.9 \\
2488.0\end{array}$ & $\begin{array}{r}593.32 * \\
1294.99 *\end{array}$ & $\begin{array}{l}50-150 \\
50-150\end{array}$ \\
\hline SURROGATE COMPOUND & $\begin{array}{l}\text { CONC } \\
\mathrm{ADDED} \\
\mathrm{ug} / \mathrm{Kg}\end{array}$ & $\begin{array}{c}\text { CONC } \\
\text { RECOVERED } \\
\text { ug/Kg }\end{array}$ & $\begin{array}{c}\% \\
\text { RECOVERED }\end{array}$ & LIMITS \\
\hline $\begin{array}{l}\$ 29 \text { Tetrachloro-m-xyle } \\
\$ 30 \text { Decachlorobiphenyl }\end{array}$ & $\begin{array}{l}192 \cdot 12 \\
192.12\end{array}$ & $\begin{array}{l}151.11 \\
110.07\end{array}$ & $\begin{array}{l}78.65 \\
57.29\end{array}$ & $\begin{array}{l}20-150 \\
20-150\end{array}$ \\
\hline
\end{tabular}




\section{2-S Laboratory \\ TARGET COMPOUNDS}

client Name:

Lab Smp Id: s97S00235MS

Sample Location:

Sample Date:

Sample Matrix: SOIL

Analysis Type: PEST

Data Type: GC MULTI COMP

Misc Info: $97 \mathrm{~S}$ 0235MS00931
Client SDG: 012398

Client Smp ID: S97S00235MS

Sample Point:

Date Received:

Quant Type: ESTD

Level: LOW

operator: Gerald Ross

CONCENTRATION UNITS :

CAS NO.

COMPOUND

( $u g / L$ or $u g / K G$ ) $u g / K g$

\begin{tabular}{|c|}
\hline $\begin{array}{l}12674-11-2----- \text { Aroclor }-1016 \\
11104-28-2----- \text { Aroclor }-1221 \\
1114-16-5-1232 \\
53469-21-9----- \text { Aroclor }-1232 \\
12672-29-6----- \text { Aroclor }-1248 \\
11097-69-1----- \text { Aroclor }-1254 \\
11096-82-5----- \text { Aroclor }-1260\end{array}$ \\
\hline $\begin{array}{l}877-09-8---1 \text { Tetrachloro-m-xylene } \\
2051-24-3-m-\infty \text { Decachlorobiphenyl }\end{array}$ \\
\hline
\end{tabular}

1139.9

670.00

330.00

330.00

8129.6

6472.1

2488 . 0

$=$

151.11

110.07

\section{U}

U

U

$==$ 


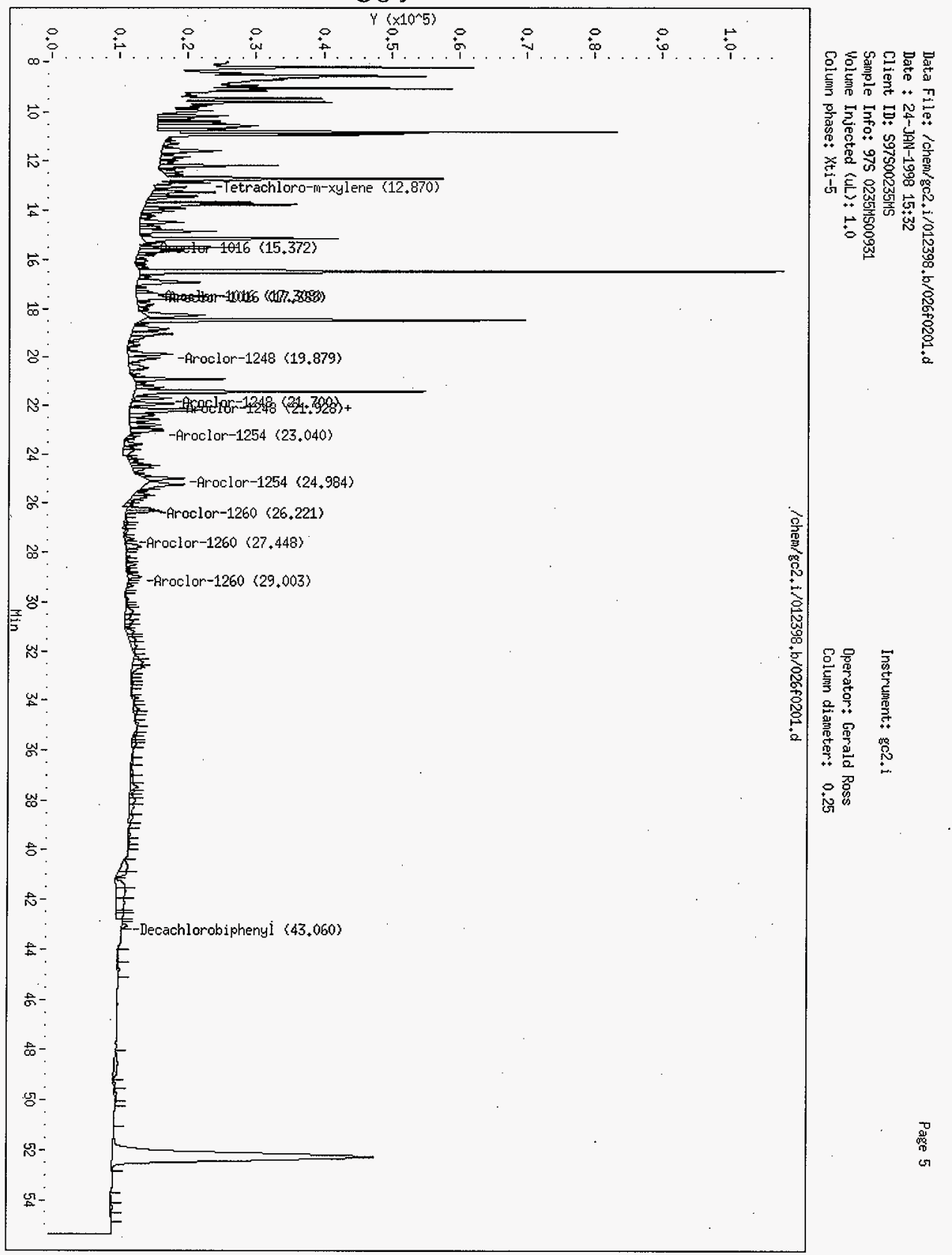


Data file : /chem/gc2.i/012398.b/027f0201.d

Lab Smp Id: S97S00235MSD

Inj Date : 24-JAN-1998 16:35

Operator : Gerald Ross

Smp Info : $97 \mathrm{~S}$ 0235MSD00933

Misc Info : $97 \mathrm{~S}$ 0235MSD00933

comment

Method

/chem/gc2.i/012398.b/rcrapcb.m

Meth Date : 27-Jan-1998 07:18 gar

Cal Date : 24-JAN-1998 05:06

Als bottle: 1

Dil Factor: 10.000

Integrator: HP Genie

Target Version: 3.10
Client Smp ID: S97S00235MSD

Inst ID: $g c 2 . i$
Quant Type: ESTD

Cal File: 016f0201.d

QC Sample: MSD

Compound Sublist: Aroclors.sub Sample Matrix: SOIL

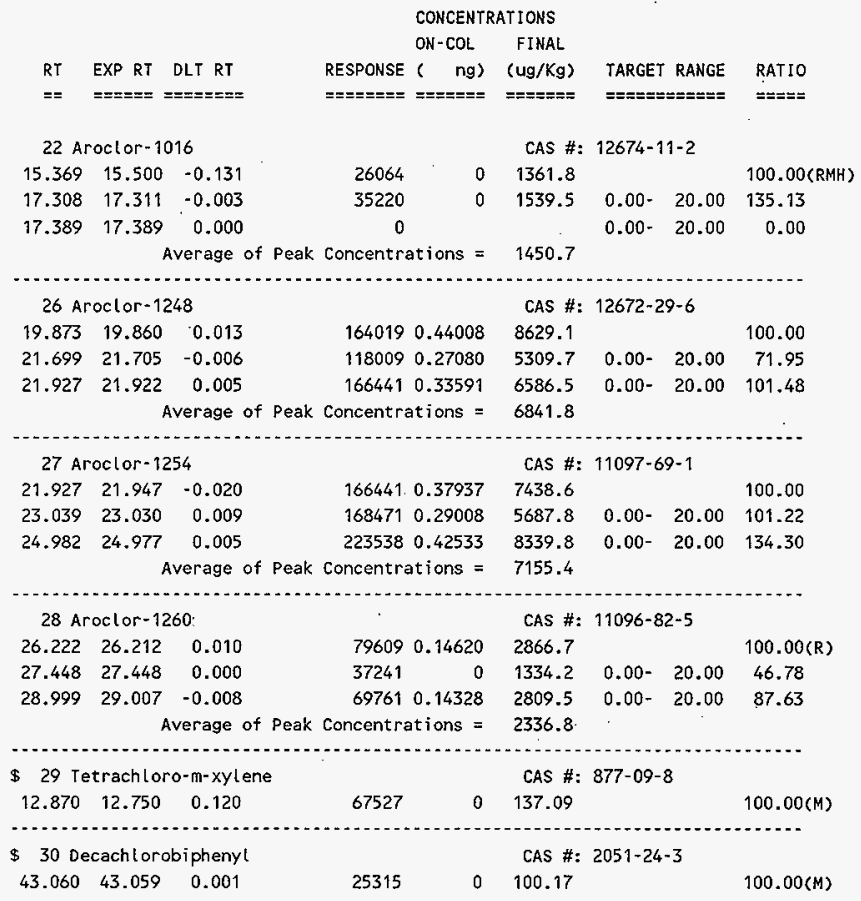


HNF-SD-WAAPP-290 REV. 0

Data File: /chem/gc2.i/012398.b/027f0201.d

Page 2

Report Date: 30-Jan-1998 13:45

QC Flag Legend

$R$ - Spike/Surrogate failed recovery limits.

M - Compound response manually integrated.

$\mathrm{H}$ - operator selected an alternate compound hit. 
Data File: /chem/gc2.i/012398.b/027f0201.d

Report Date: 30-Jan-1998 13:45

\section{2-S Laboratory}

RECOVERY REPORT

Client Name:

Sample Matrix: Solid

Lab Smp Id: S97S00235MSD

Level: LOW

Data Type: GC MULTI COMP

Spikelist File: Aroclor-1660.spk

Method

Misc Info: $97 \mathrm{~S} 0235 \mathrm{MSD} 00933$
Client SDG: 012398

Fraction: PEST

Client Smp ID: S97S00235MSD

Operator: Gerald Ross

SampleType: MSD

Quant Type: ESTD

\begin{tabular}{|c|c|c|c|c|}
\hline SPIKE COMPOUND & $\begin{array}{l}\text { CONC } \\
\text { ADDED } \\
\mathrm{ug} / \mathrm{Kg}\end{array}$ & $\begin{array}{c}\text { CONC } \\
\text { RECOVERED } \\
\text { ug/Kg }\end{array}$ & $\begin{array}{c}\stackrel{\circ}{\circ} \\
\text { RECOVERED }\end{array}$ & LIMITS \\
\hline $\begin{array}{l}22 \text { Aroclor }-1016 \\
28 \text { Aroclor }-1260\end{array}$ & $\begin{array}{l}196.08 \\
196.08\end{array}$ & $\begin{array}{l}1450.7 \\
2336.8\end{array}$ & $\begin{array}{r}739.85 * \\
1191.76 *\end{array}$ & $\begin{array}{l}50-150 \\
50-150\end{array}$ \\
\hline . & & & & \\
\hline SURROGATE COMPOUND & $\begin{array}{l}\text { CONC } \\
\text { ADDED } \\
\mathrm{ug} / \mathrm{Kg}\end{array}$ & $\begin{array}{c}\text { CONC } \\
\text { RECOVERED } \\
\text { ug/Kg }\end{array}$ & RECOVERED & LIMITS \\
\hline $\begin{array}{l}\$ 29 \text { Tetrachloro-m-xyle } \\
\$ \quad 30 \text { Decachlorobiphenyl }\end{array}$ & $\begin{array}{l}196.08 \\
196.08\end{array}$ & $\begin{array}{l}137.09 \\
100.17\end{array}$ & $\begin{array}{l}69.91 \\
51.09\end{array}$ & $\begin{array}{l}\overline{20-150} \\
20-150\end{array}$ \\
\hline
\end{tabular}


Data File: /chem/gc2.i/012398.b/027f0201.d

Report Date: 30-Jan-1998 13:45

\section{2-S Laboratory}

TARGET COMPOUNDS

client Name:

Lab Smp Id: S97S00235MSD

Sample Location:

Sample Date:

Sample Matrix: SOIL

Analysis Type: PEST

Data Type: GC MULTI COMP

Misc Info: $97 \mathrm{~S}$ 0235MSD00933
Client SDG: 012398

Client Smp ID: S97S00235MSD

Sample Point:

Date Received:

Quant Type: ESTD

Level: LOW

Operator: Gerald Ross

CAS NO.

COMPOUND

CONCENTRATION UNITS:

(ug/L or $\mathrm{ug} / \mathrm{KG}$ ) ug/Kg

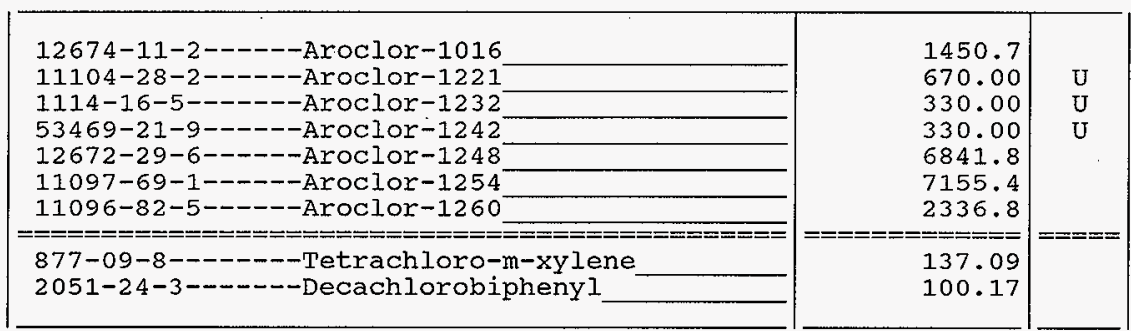




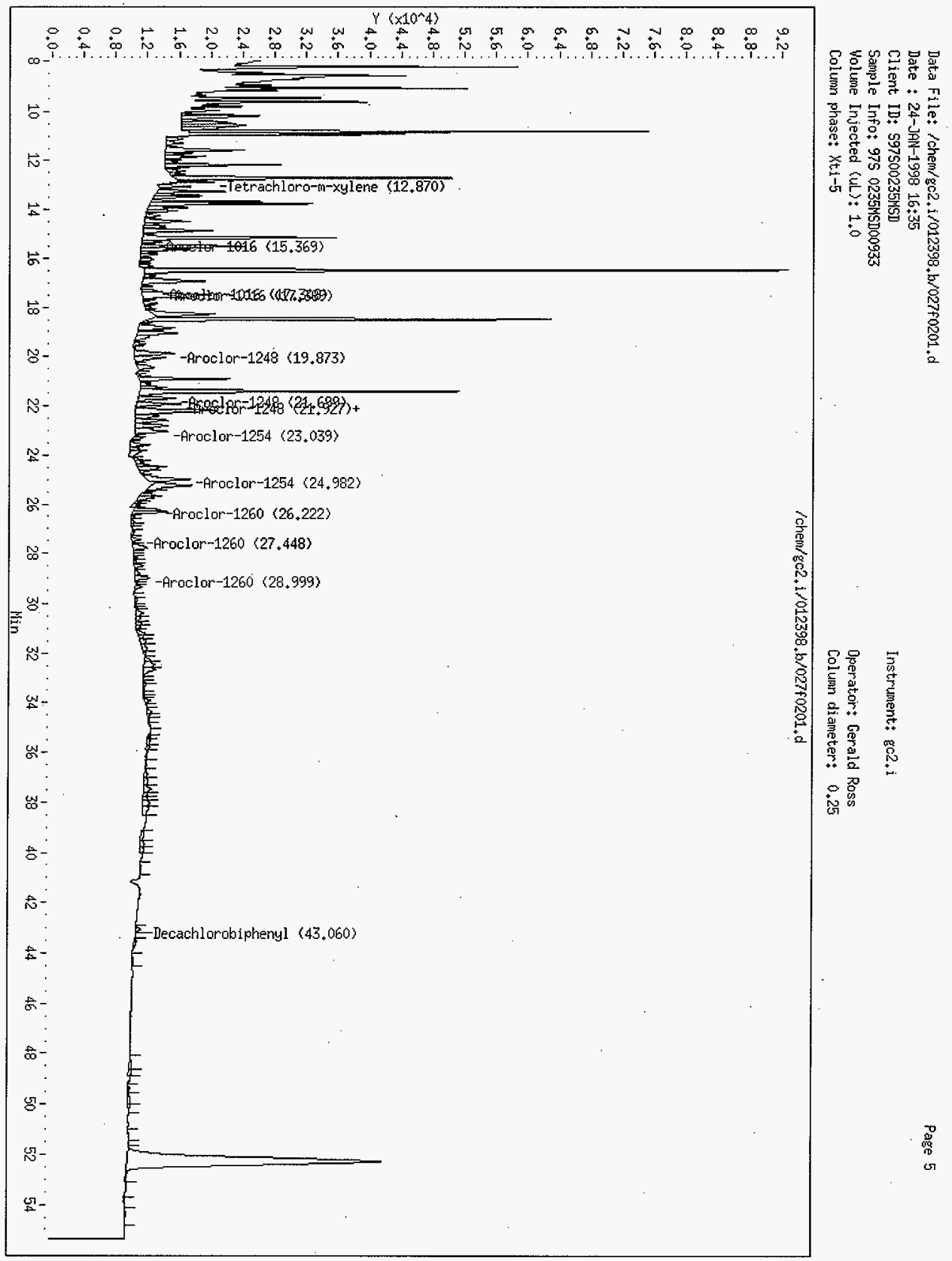




\section{HNF-SD-WM-DP-290 REV. 0}

Data File: /chem/gc2.i/012398.b/028f0201.d

Report Date: 30-Jan-1998 11:54

Page 1

\section{2-S Laboratory}

Data file : /chem/gc2.i/012398.b/028f0201.d

Iab smp Id: inst. blnk

Inj Date : 24-JAN-1998

operator : Gerald Ross

Smp Info : inst. blnk

Misc Info : inst. blnk

Comment

Method

Meth Date

Cal Date

Als bottle:

Dil factor: 1

Integrator: HP Genie

Target Version: 3.10 client Smp ID: inst. blnk

Inst ID: gc2.i /chem/gc2.i/012398.b/rcrapcb.m

$$
\text { 27-Jan-1998 07:18 gar }
$$

24-JAN-1998 05:06

1

1.000
Quant Type: ESTD

Cal File: 016 $0201 . d$

Compound Sublist: Aroclors.sub Sample Matrix: SOIL

CONCENTRATIONS

ON-COL FINAL

RT EXP RT DLJ RT RESPONSE ( $\mathrm{ng}) \cdot(\mathrm{ug} / \mathrm{Kg})$ TARGET RANGE RAJIO

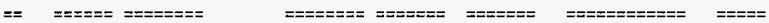

22 Aroclor -1016

CAS \#: $12674-11-2$

Peaks not detected for Quant. or Qual. signal(s).

Peaks not detected for Quant. or Qual. signal(s).

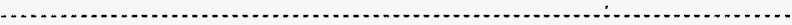

24 Aroclor -1232

CAS \# $1114-16-5$

Peaks not detected for Quant. or Qual. signal(s).

25 Aroclor -1242

CAS \#: $53469-21-9$

Peaks not detected for Quant. or Qual. signal(s).

Peaks not detected for Quant. or Qual. signal(s).

Peaks not detected for Quant. or Qual. signal(s). 
HNF-SD-WAADP-290 REV. 0

Data File: /chem/gc2, i/012398.b/028f0201.d

Report Date: 30-Jan-1998 11:54

\begin{tabular}{|c|c|c|c|c|c|c|}
\hline \multirow[t]{2}{*}{, } & & & \multicolumn{4}{|c|}{ CONCENTRATIONS } \\
\hline & & & $\mathrm{ON}-\mathrm{COL}$ & FINAL & & \\
\hline $\mathbf{R T}$ & EXP RT DLT RT & RESPONSE & ( $\mathrm{ng})$ & $(u g / K g)$ & TARGET RANGE & RAIIO \\
\hline$==$ & 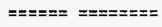 & $=====-==$ & $= \pm== \pm==$ & Iニニニニニニ & 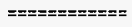 & $====-$ \\
\hline & oclor- 1260 & & & CAS \# & $11096-82-5$ & \\
\hline
\end{tabular}

Peaks not detected for Quant. or Qual. signal( $(s)$.

$\$ 29$ Tetrachloro-m-xylene

CAS \#: $877-09-8$

$\begin{array}{llllll}12.748 & 12.750 & -0.002 & 552391 & 0 & 114.38\end{array}$

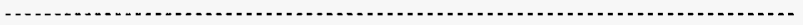

$\$ 30$ Decachlorobiphenyl

CAS \#: $2051-24-3$

$\begin{array}{llll}43.058 & 43.059 & -0.001\end{array}$

239918

$0 \quad 96.832$

100.00 


\section{2-S Laboratory}

\section{RECOVERY REPORT}

Client Name:

Sample Matrix: SoLID

Lab smp Id: inst. blnk

Level: LOW

Data Type: GC MULTI COMP

SpikeList File: RESOLUTION. spk

Method File: / chem/gc2.i/012398.b/rcrapcb.m

Misc Info: inst. blnk
Client SDG: 012398

Fraction: PEST

Client Smp ID: inst. blnk

operator: Gerald Ross

SampleType: SAMPLE

Quant Type: ESTD

\begin{tabular}{|c|c|c|c|c|}
\hline SURROGATE COMPOUND & $\begin{array}{l}\text { CONC } \\
\text { ADDED }\end{array}$ & CONC & RECOVERED & LIMITS \\
\hline $\begin{array}{lll}\$ 29 & \text { Tetrachloro-m-xyle } \\
\$ & 30 \text { Decachlorobiphenyl }\end{array}$ & $\begin{array}{l}104.00 \\
104.00\end{array}$ & $\begin{array}{l}114.38 \\
96.832\end{array}$ & $\begin{array}{r}109.98 \\
93.11\end{array}$ & $\begin{array}{l}20-150 \\
20-150\end{array}$ \\
\hline
\end{tabular}




\section{2-S Laboratory}

TARGET COMPOUNDS

Client Name:

Lab Smp Id: inst. blnk

Sample Location:

Sample Date:

Sample Matrix: SoIL

Analysis Type: PEST

Data Type: GC MULTI COMP

Misc Info: inst. blnk
Client SDG: 012398

Client Smp ID: inst. blnk

Sample Point:

Date Received:

Quant Type: ESTD

Level: LOW

Operator: Gerald Ross

CONCENTRATION UNITS: $(\mathrm{ug} / \mathrm{L}$ or $\mathrm{ug} / \mathrm{KG}) \mathrm{ug} / \mathrm{Kg}$

\begin{tabular}{|c|c|c|}
\hline $\begin{array}{l}12674-11-2----- \text { Aroclor }-1016 \\
11104-28-2---- \text { Aroclor }-1221 \\
1114-16-5---- \text { Aroclor }-1232 \\
53469-21-9---- \text { Aroclor }-1242 \\
12672-29-6----- \text { Aroclor }-1248 \\
110.97-69-1----- \text { Aroclor }-1254 \\
11096-82-5----- \text { Aroclor }-1260\end{array}$ & $\begin{array}{l}33.000 \\
67.000 \\
33.000 \\
33.000 \\
33.000 \\
3.7100 \\
33.000\end{array}$ & $\begin{array}{l}\mathrm{U} \\
\mathrm{U} \\
\mathrm{U} \\
\mathrm{U} \\
\mathrm{U} \\
\mathrm{U} \\
\mathrm{U}\end{array}$ \\
\hline $\begin{array}{l}877-09-8-10-\text { Tetrachloro-m-xylene } \\
2051-24-3-\end{array}$ & $\begin{array}{l}114.38 \\
96.832\end{array}$ & \\
\hline
\end{tabular}


HNF-SD-WADP-290 REV. 0

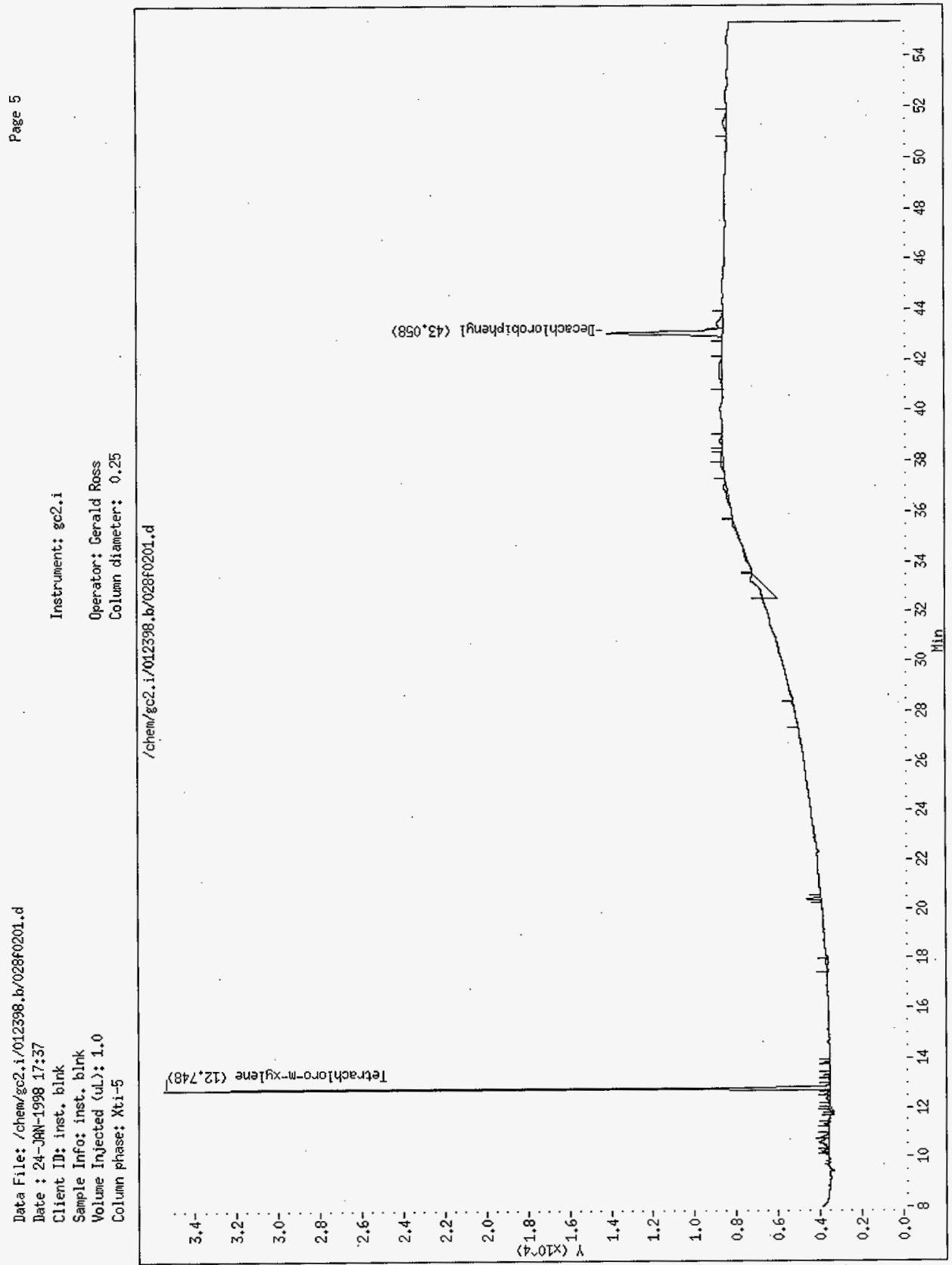


HNF-SD-WM-DP-290, REV. 0

CONTINUING CALIBRATION VERIFICATION 
HNF-SD-HM-DP-290, REV. 0

THIS PAGE INTENTIONALLY LEFT BLANK 


\title{
222-S Laboratory
}

\author{
Data file : /chem/gc2.i/012398.b/029f0201.d \\ Lab Smp Id: 100ng/ml A1660 \\ Client Smp ID: $100 \mathrm{ng} / \mathrm{ml}$ A1660 \\ Inj Date : 24-JAN-1998 18:40 \\ operator : Gerald Ross \\ Smp Info : 100 ng/ml A1660 \\ Inst ID: $g \mathrm{c} 2 . \mathrm{i}$ \\ Misc Info : 100ng/ml A1660 \\ comment \\ Method \\ /chem/gc2.i/012398.b/rcrapcb.m \\ Meth Date : 27-Jan-1998 07:18 gar \\ Cal Date : 24-JAN-1998 05:06 \\ Quant Type: ESTD \\ Als bottle: 1 \\ Dil Factor: 1.000 \\ Integrator: HP Genie \\ Target Version: 3.10 \\ Cal File: 016f0201.d \\ Compound Sublist: Aroclors. sub \\ Sample Matrix: SoIL
}

\begin{tabular}{|c|c|c|c|c|c|c|c|}
\hline & & & & CONCENTR & AI IONS & & \\
\hline & & & & $\mathrm{ON}-\mathrm{COL}$ & FINAL & & \\
\hline RT & EXP RT & DLT RT & RESPONSE & ( $n g)$ & $(u g / K g)$ & TARGET RANGE & RATIO \\
\hline$=$ & $m=====$ & $==ニ==ニ==$ & 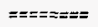 & $==ニ===0$ & $==ニ= \pm==$ & 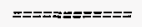 & $==\pi==$ \\
\hline $22 \mathrm{Ar}$ & roclor-10 & 1016 & & & CAS \#: & $12674-11-2$ & \\
\hline 15.497 & 15.500 & -0.003 & 46220 & 0.12316 & 123.16 & & 100.00 \\
\hline 17.305 & 17.311 & -0.006 & 60307 & 0.13444 & 134.44 & $0.00-20.00$ & 130.48 \\
\hline 17.385 & 17.389 & -0.004 & 69481 & 0.12592 & 125.92 & $0.00-20.00$ & 150.33 \\
\hline & & Average of Peak & Concentra & ations $=$ & 127.84 & & \\
\hline $28 \mathrm{Ar}$ & roclor-12 & 260 & & & CAS \#: & $11096-82-5$ & \\
\hline 26.208 & 26.212 & -0.004 & 64000 & 0.11754 & 117.54 & & 100.00 \\
\hline 27.445 & 27.448 & -0.003 & 63915 & 0.11678 & 116.78 & $0.00-20.00$ & 99.87 \\
\hline 29.002 & 29.007 & -0.005 & 54516 & 0.11197 & 111.97 & $0.00-\quad 20.00$ & 85.18 \\
\hline & & Average of Peak & Concentra & ations = & 115.43 & & \\
\hline $29 \mathrm{Je}$ & etrachlor & ro-m-xyl ene & & & CAS \#: & $877-09-8$ & \\
\hline 12.746 & 12.750 & -0.004 & 642341 & 0 & 66.505 & & 100.00 \\
\hline $30 \mathrm{De}$ & ecachloro & obiphenyl & & & CAS \#: & $2051-24-3$ & \\
\hline 43.056 & 43.059 & -0.003 & 359872 & 0 & 72.623 & & 100.00 \\
\hline
\end{tabular}




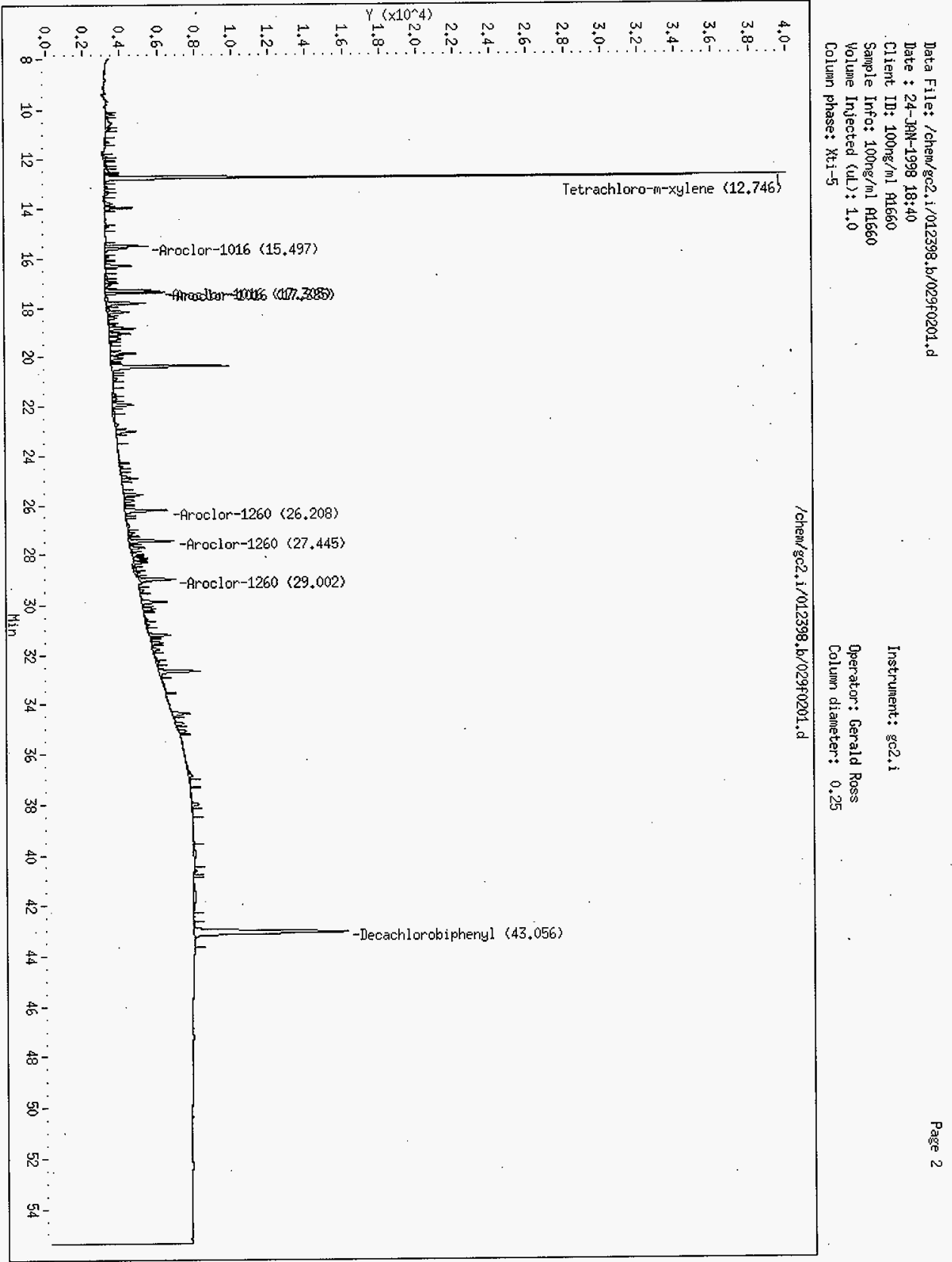


Data file : /chem/gc2.i/012398.b/036f0201.d
Lab Smp Id: 100 A1660
Inj Date : 25-JAN-1998 01:58
operator : Gerald Ross
Client Smp ID: 100 A1660
Smp Info : 100 A 1660
Inst ID: $g \mathrm{C} 2 . i$
Misc Info : $100 \mathrm{~A} 1660$
Comment
Method : /chem/gc2.i/012398.b/rcrapcb.m
Meth Date : 27-Jan-1998 07:18 gar Quant Type: ESTD
Cal Date : 24-JAN-1998 05:06 Cal File: 016f0201.d
Als bottle: 1
Dil Factor: 1.000
Integrator: HP Genie
Target Version: 3.10
Compound Sublist: Aroclors. sub Sample Matrix: SoII

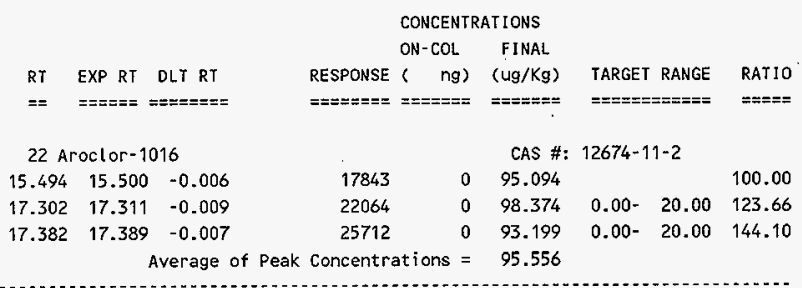

23 Aroclor -1221

CAS \#: $11104=28-2$

Peaks not detected for Quant. or Qual. signal(s).

24 Aroclor $-1232 \quad$ CAS \#: $1114-16-5$

Peaks not detected for Quant. or Qual. signal(s).

25 Araclor $-1242 \quad$ CAS \#: 53469-21-9

Peaks not detected for Quant. or Qual. signal(s).

Peaks not detected for Qunt. or Qual. signal(s)

26 Aroclor -1248

CAS \#: $12672-29-6$

Peaks not detected for Quant. or Qual. signal(s).

\begin{tabular}{|c|c|c|c|c|c|c|c|c|}
\hline \multicolumn{4}{|c|}{27 Aroclor -1254} & \multicolumn{5}{|c|}{ CAS \#: $11097-69-1$} \\
\hline 21.937 & 21.947 & -0.010 & 12944 & 0 & 59.006 & & & 100.00 \\
\hline 23.014 & 23.030 & -0.016 & 9675 & 0 & 33.317 & $0.00-$ & 20.00 & 74.75 \\
\hline 24.964 & 24.977 & -0.013 & 4000 & 0 & 15.222 & $0.00-$ & 20.00 & 30.90 \\
\hline
\end{tabular}


HNF-SD-WADP-290 REV. 0

Data File: /chem/gc2.i/012398.b/036f0201.d

Report Date: 02-Feb-1998 08:57

\begin{tabular}{|c|c|c|c|c|c|c|c|}
\hline & & & & CONCENTR & ATIONS & & \\
\hline & & & & $\mathrm{ON}-\mathrm{COL}$ & FINAL & & \\
\hline RT & EXP RT & DLT RT & RESPONSE & ( $\quad n g)$ & (ug/Kg) & TARGET RANGE & RATIO \\
\hline$==$ & $== \pm= \pm==$ & $=== \pm====$ & $=======$ & 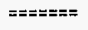 & $==== \pm= \pm$ & $=====0===$ & $====$ \\
\hline $28 \mathrm{Ar}$ & roclor -12 & 260 & & & CAS \#: & $11096-82-5$ & \\
\hline 26.205 & 26.212 & -0.007 & 22956 & 0 & 84.318 & & 100.00 \\
\hline 27.441 & 27.448 & -0.007 & 22651 & 0 & 82.771 & $0.00-20.00$ & 98.67 \\
\hline 28.999 & 29.007 & -0.008 & 18841 & 0 & 77.395 & $0.00-20.00$ & 82.07 \\
\hline & & Average of & Concentra & ations = & 81.495 & & \\
\hline $29 \mathrm{TC}$ & etrachlor & ro-m-xyle & & & CAS \#: & $877-09-8$ & \\
\hline 12.744 & 12.750 & -0.006 & 457232 & 0 & 94.679 & & 100.00 \\
\hline $30 \mathrm{De}$ & ecach loro & obiphenyl & & & CAS \#: & $2051-24-3$ & \\
\hline 43.048 & 43.059 & -0.011 & 166736 & 0 & 67.295 & & 100.00 \\
\hline
\end{tabular}




\begin{tabular}{|c|c|c|c|c|}
\hline \multicolumn{5}{|c|}{ DISTRIBUTION SHEET } \\
\hline \multirow{2}{*}{$\begin{array}{l}\text { To } \\
\text { Distribution }\end{array}$} & \multirow[t]{2}{*}{ From } & \multirow{2}{*}{\multicolumn{2}{|c|}{ Production Planning \& Control }} & Page 1 of 1 \\
\hline & & & & Date: $02 / 03 / 98$ \\
\hline \multirow{2}{*}{\multicolumn{4}{|c|}{ 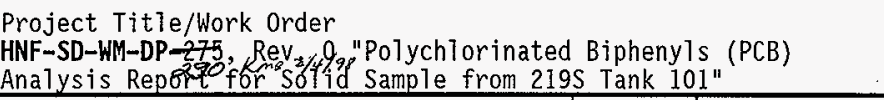 }} & EDT N0.: EDT-623140 \\
\hline & & & & ECN NO.: N/A \\
\hline & Name & MSIN & $\begin{array}{l}\text { Text With } \\
\text { a11 Attach }\end{array}$ & $\begin{array}{l}\text { EDT/ECN } \\
\text { ONLY }\end{array}$ \\
\hline
\end{tabular}

U.S. Department of Energy

E. M. Mattlin

A5-15 $X$

Lockheed Martin Hanford Corp.

B. G. Erlandson

C. H. Mulkey

R1-51 $\quad x$

Fluor Daniel Hanford, Inc.

T. A. Quayle

Lockheed Martin Services, Inc.

\section{Centra] Files}

Los Alamos Technical Associates

M. T. El] sworth

Waste Management of Hanford, Inc.

J. B. Buckley

R. H. Engelmann

L. P. Markel

L. F. Perkins

J. R. Prilucik

G. A. Ross

C. M. Seide1

K. S. Tollefson

J. A. Winterhalder
H6-23

$x$

R1-51 $\quad X$

A3-88 $\quad 1$

T6-06 $\quad X^{*}$

$\begin{array}{ll}\text { T3-04 } & X \\ H 6-26 & X \\ \text { T6-16 } & X \\ \text { T6-14 } & X \\ T 6-14 & X \\ \text { T6-50 } & X \\ T 6-14 & X \\ \text { T6-12 } & X \\ \text { H6-21 } & X\end{array}$

* Needs only releasing paperwork, not a copy of the released document. 